NBER WORKING PAPER SERIES

\title{
THE MACROECONOMIC IMPACT OF MICROECONOMIC SHOCKS: BEYOND HULTEN'S THEOREM
}

\author{
David Rezza Baqaee \\ Emmanuel Farhi \\ Working Paper 23145 \\ http://www.nber.org/papers/w23145 \\ NATIONAL BUREAU OF ECONOMIC RESEARCH \\ 1050 Massachusetts Avenue \\ Cambridge, MA 02138 \\ February 2017
}

We provide a nonlinear characterization of the macroeconomic impact of microeconomic productivity shocks in terms of reduced-form non-parametric elasticities for efficient economies. We also show how microeconomic parameters are mapped to these reduced-form general equilibrium elasticities. In this sense, we extend the foundational theorem of Hulten (1978) beyond the first order to capture nonlinearities. Key features ignored by first-order approximations that play a crucial role are: structural microeconomic elasticities of substitution, network linkages, structural microeconomic returns to scale, and the extent of factor reallocation. In a business-cycle calibration with sectoral shocks, nonlinearities magnify negative shocks and attenuate positive shocks, resulting in an aggregate output distribution that is asymmetric (negative skewness), fat-tailed (excess kurtosis), and a has negative mean, even when shocks are symmetric and thin-tailed. Average output losses due to short-run sectoral shocks are an order of magnitude larger than the welfare cost of business cycles calculated by Lucas (1987). Nonlinearities can also cause shocks to critical sectors to have disproportionate macroeconomic effects, almost tripling the estimated impact of the 1970s oil shocks on world aggregate output. Finally, in a long-run growth context, nonlinearities, which underpin Baumol's cost disease via the increase over time in the sales shares of low-growth bottleneck sectors, account for a 20 percentage point reduction in aggregate TFP growth over the period 1948-2014 in the US. The views expressed herein are those of the authors and do not necessarily reflect the views of the National Bureau of Economic Research.

NBER working papers are circulated for discussion and comment purposes. They have not been peer-reviewed or been subject to the review by the NBER Board of Directors that accompanies official NBER publications.

(C) 2017 by David Rezza Baqaee and Emmanuel Farhi. All rights reserved. Short sections of text, not to exceed two paragraphs, may be quoted without explicit permission provided that full credit, including $\odot$ notice, is given to the source. 
The Macroeconomic Impact of Microeconomic Shocks: Beyond Hulten's Theorem

David Rezza Baqaee and Emmanuel Farhi

NBER Working Paper No. 23145

February 2017

JEL No. E01,E1,E23,E32,L16

\begin{abstract}
$\underline{\text { ABSTRACT }}$
We provide a nonlinear characterization of the macroeconomic impact of microeconomic productivity shocks in terms of reduced-form non-parametric elasticities for efficient economies. We also show how microeconomic parameters are mapped to these reduced-form general equilibrium elasticities. In this sense, we extend the foundational theorem of Hulten (1978) beyond the first order to capture nonlinearities. Key features ignored by first-order approximations that play a crucial role are: structural microeconomic elasticities of substitution, network linkages, structural microeconomic returns to scale, and the extent of factor reallocation. In a business-cycle calibration with sectoral shocks, nonlinearities magnify negative shocks and attenuate positive shocks, resulting in an aggregate output distribution that is asymmetric (negative skewness), fat-tailed (excess kurtosis), and a has negative mean, even when shocks are symmetric and thin-tailed. Average output losses due to short-run sectoral shocks are an order of magnitude larger than the welfare cost of business cycles calculated by Lucas (1987). Nonlinearities can also cause shocks to critical sectors to have disproportionate macroeconomic effects, almost tripling the estimated impact of the 1970s oil shocks on world aggregate output. Finally, in a long-run growth context, nonlinearities, which underpin Baumol's cost disease via the increase over time in the sales shares of low-growth bottleneck sectors, account for a 20 percentage point reduction in aggregate TFP growth over the period 1948-2014 in the US.
\end{abstract}

\author{
David Rezza Baqaee \\ UCLA \\ 315 Portola Plaza \\ Los Angeles \\ baqaee@econ.ucla.edu \\ Emmanuel Farhi \\ Harvard University \\ Department of Economics \\ Littauer Center \\ Cambridge, MA 02138 \\ and NBER \\ emmanuel.farhi@gmail.com
}




\section{Introduction}

The foundational theorem of Hulten (1978) states that for efficient economies and under minimal assumptions, the impact on aggregate TFP of a microeconomic TFP shock is equal to the shocked producer's sales as a share of GDP:

$$
\mathrm{d} \log T F P=\sum_{i} \lambda_{i} \mathrm{~d} \log A_{i},
$$

where $\mathrm{d} \log A_{i}$ is a shock to producer $i$ and $\lambda_{i}$ is its sales share or Domar weight.

Hulten's theorem is a cornerstone of productivity and growth accounting: it shows how to construct aggregate TFP growth from microeconomic TFP growth, and provides structurally-interpretable decompositions of changes of national or sectoral aggregates into the changes of their disaggregated component industries or firms. It also provides the benchmark answers for counterfactual questions in structural models with disaggregated production.

The surprising generality of the result has led economists to de-emphasize the role of microeconomic and network production structures in macroeconomic models. After all, if sales summarize the macroeconomic impact of microeconomic shocks and we can directly observe sales, then we need not concern ourselves with the details of the underlying disaggregated system that gave rise to these sales. Since it seems to imply that the very object of its study is irrelevant for macroeconomics, Hulten's theorem has been something of a bugbear for the burgeoning literature on production networks.

Are these conclusions warranted? Even at a purely intuitive level, there are reasons to be skeptical. Take for example shocks to Walmart and to electricity production. Both Walmart and electricity production have a similar sales share of roughly $4 \%$ of U.S. GDP. It seems natural to expect that a large negative shock to electricity production would be much more damaging than a similar shock to Walmart. Indeed, this intuition will be validated by our formal results. Yet it goes against the logic of Hulten's theorem which implies that, because the two sectors have the same Domar weight, the two shocks should have the same impact on aggregate output.

In this paper, we challenge the view that the macroeconomic importance of a microeconomic sector is summarized by its sales share and, more broadly, the notion that the microeconomic details of the production structure are irrelevant for macroeconomics. The key is to recognize that Hulten's theorem only provides a first-order approximation. Nonlinearities can significantly degrade the quality of the first-order approximation for large enough shocks. To capture these nonlinearities, we provide a general second-order approximation by characterizing the derivatives of Domar weights with respect to shocks. The 
second-order terms are shaped by the microeconomic details of the disaggregated production structure: network linkages, microeconomic elasticities of substitution in production, microeconomic returns to scale, and the degree to which factors can be reallocated.

Our results are general in that they apply to any efficient general equilibrium economy. They suggest that Cobb-Douglas models, commonly used in the production-network, growth, and multi-sector macroeconomics literatures, are very special: the Domar weights, and more generally the whole input-output matrix, are constant and can be taken to be exogenous, the first-order approximation is exact, the model is log-linear, and as a result, the microeconomic details of the production structure are irrelevant. ${ }^{1}$ These knife-edge properties disappear as soon as one deviates from Cobb-Douglas: the Domar weights and more generally the whole input-output matrix respond endogenously to shocks, and the resulting nonlinearities are shaped by the microeconomic details of the production structure.

We also show that nonlinearities in production matter quantitatively for a number of macroeconomic phenomena operating at different frequencies, ranging from the role of sectoral shocks in business cycles to the impact of oil shocks and the importance of Baumol's cost disease for long-run growth:

1. Using a calibrated structural multi-industry model with realistic complementarities in production, we find that nonlinearities amplify the impact of negative sectoral shocks and mitigate the impact of positive sectoral shocks. ${ }^{2,3}$ Large negative shocks to crucial industries, like "oil and gas", have a significantly larger negative effect on aggregate output than negative shocks to larger but less crucial industries such as "retail trade". Nonlinearities also have a significant impact on the distribution of aggregate output: they lower its mean and generate negative skewness and excess kurtosis even though the underlying shocks are symmetric and thin tailed. Nonlinearities in production generate significant welfare costs of sectoral fluctuations, ranging from $0.2 \%$ to $1.3 \%$ depending on the calibration. These are an order of magnitude larger than the welfare

\footnotetext{
${ }^{1}$ A mixture of analytical tractability, as well as balanced-growth considerations, have made Cobb-Douglas the canonical production function for networks (Long and Plosser, 1983), multisector RBC models (Gomme and Rupert, 2007), and growth theory (Aghion and Howitt, 2008). Recent work by Grossman et al. (2016) shows how balanced growth can occur without Cobb-Douglas.

${ }^{2}$ The empirical literature on production networks, like Atalay (2017), Boehm et al. (2017), and Barrot and Sauvagnat (2016) all find that structural elasticities of substitution in production are significantly below one, and sometimes very close to zero, across intermediate inputs, and between intermediate inputs and labor at business cycle frequencies. Furthermore, a voluminous literature on structural transformation, building on Baumol (1967), has found evidence in favor of non-unitary elasticities of substitution in consumption and production across sectors over the long-run.

${ }^{3}$ While complementarities prevail at the sectoral level, substitutabilities dominate across firms within sectors. This implies that while nonlinearities tend to amplify negative sectoral-level shocks and to attenuate positive sectoral-level shocks, they tend to attenuate negative firm-level shocks and to amplify positive firmlevel shocks. Nonlinearities therefore introduce an important qualitative difference between sectoral- and firm-level shocks which is absent from the linearized perspective.
} 
costs of business cycles arising from nonlinearities in utility (risk aversion) identified by Lucas (1987).

2. We derive and use a simple nonparametric formula, taking into account the observed change in the Domar weight for crude oil, to analyze the impact of the energy crisis of the 1970s up to the second order. We find that nonlinearities almost tripled the impact of the oil shocks from $0.23 \%$ to $0.61 \%$ of world aggregate output.

3. We show that the nonlinearities are also important for long-run growth in the presence of realistic complementarities across sectors. They cause the Domar weights of bottleneck sectors with relatively low productivity growth to grow over time and thereby reduce aggregate growth, an effect identified as Baumol's cost disease (Baumol, 1967). We calculate that nonlinearities have reduced the growth of aggregate TFP by 20 percentage points over the period 1948-2014 in the US. ${ }^{4}$

The outline of the paper is as follows. In Section 2, we derive a general formula describing the second-order impact on aggregate output of shocks in terms of non-parametric sufficient statistics: reduced-form general-equilibrium elasticities of substitution and input-output multipliers. ${ }^{5}$ We explain the implications of this formula for the impact of correlated shocks and for the average performance of the economy. In Section 3, we use two special illustrative examples to provide some intuition for the roles of the general-equilibrium elasticities of substitution and of the input-output multipliers and for their dependence on microeconomic primitives. In Section 4, we fully characterize second-order terms in terms of microeconomic primitives for general nested-CES economies with arbitrary microeconomic elasticities of substitution and network linkages. In Section 5, we further generalize the results to arbitrary (potentially non-CES) production functions. In Section 6, we provide some illustrations of the quantitative implications of our results.

Related literature. Gabaix (2011) uses Hulten's theorem to argue that the existence of very large, or in his language granular firms, can be a possible source of aggregate volatility. If

\footnotetext{
${ }^{4}$ The literature on structural transformation emphasizes two key forces: non-unitary elasticities of substitution and non-homotheticities. Both forces cause sales shares to change in response to exogenous shocks. Since Hulten's theorem implies that sales shares are equal to derivatives of the aggregate output function, anything that causes the derivative to change is a non-linearity. By characterizing the second-order terms in a general way, our results encompass both non-unitary elasticities and non-homotheticities. In fact, non-homotheticities can always be turned into non-unitary elasticities of substitution by adding more fixed-factors to an economy.

${ }^{5}$ Studying the second-order terms is the first step in grappling with the nonlinearities inherent in multisector models with production networks. In this sense, our work illustrates the macroeconomic importance of local and strongly nonlinear interactions emphasized in reduced form by Scheinkman and Woodford (1994). Other related work on nonlinear propagation of shocks in economic networks includes Durlauf (1993), Jovanovic (1987), Ballester et al. (2006) Acemoglu et al. (2015), Elliott et al. (2014), and especially Acemoglu et al. (2016).
} 
there exist very large firms, then shocks to those firms will not cancel out with shocks to much smaller firms, resulting in aggregate fluctuations. Acemoglu et al. (2012), working with a Cobb-Douglas model in the spirit of Long and Plosser (1983), observed that in an economy with input-output linkages, the equilibrium sizes of firms depend on the shape of the input-output matrix. Central suppliers will be weighted more highly than peripheral firms, and therefore, shocks to those central players will not cancel out with shocks to small firms. ${ }^{6}$ Carvalho and Gabaix (2013) show how Hulten's theorem can be operationalized to decompose the sectoral sources of aggregate volatility. ${ }^{7}$

Relatedly, Acemoglu et al. (2017) deploy Hulten's theorem to study other moments of the distribution of aggregate output. They argue that if the Domar weights are fattailed and if the underlying idiosyncratic shocks are fat-tailed, then aggregate output can exhibit non-normal behavior. Stated differently, they show that aggregate output can inherit tail risk from idiosyncratic tail risk if the distribution of the Domar weights is fat-tailed. Our paper makes a related but distinct point. We find that, for the empirically relevant range of parameters, the response of aggregate output to shocks is significantly asymmetric. Therefore, the nonlinearity inherent in the production structure can turn even symmetric thin-tailed sectoral shocks into rare disasters endogenously. This means that the economy could plausibly experience aggregate tail risk without either fat-tailed shocks or fat-tailed Domar weights.

In a recent survey article Gabaix (2016), invoking Hulten's theorem, writes "networks are a particular case of granularity rather than an alternative to it." This has meant that researchers studying the role of networks have either moved away from efficient models, or that they have retreated from studying aggregate output and turned their attention to the microeconomic implications of networks, namely the covariance of fluctuations between different industries and firms. ${ }^{8,9}$ However, our paper shows that except in very special cases, models with the same sales distributions but different network structures only have the same

\footnotetext{
${ }^{6} \mathrm{~A}$ related version of this argument was also advanced by Horvath (1998), who explored this issue quantitatively with a more general model in Horvath (2000). Separately, Carvalho (2010) also explores how the law of large numbers may fail under certain conditions on the input-output matrix.

${ }^{7}$ Results related to Hulten's theorem are also used in international trade, e.g. Burstein and Cravino (2015), to infer the global gains from international trade.

${ }^{8}$ Some recent papers have investigated aggregate volatility in production networks with inefficient equilibria (where Hulten's theorem does not hold). Some examples include Bigio and La'O (2016), Baqaee (2018), Altinoglu (2016), Grassi (2017), and Baqaee and Farhi (2018c). See also Jones (2011), Jones (2013), Bartelme and Gorodnichenko (2015), and Liu (2017).

${ }^{9}$ Other papers investigate the importance of idiosyncratic shocks propagating through networks to generate cross-sectional covariances, but refrain from analyzing aggregate output. Some examples include Foerster et al. (2011), Atalay (2017), Di Giovanni et al. (2014), and Stella (2015), and Baqaee and Farhi (2018b). Atalay (2017) is particularly relevant in this context, since he finds that structural elasticities of substitution in production play a powerful role in generating covariance in sectoral output. Our paper complements this analysis by focusing instead on the way complementarities affect aggregate output.
} 
aggregate-output implications up to a fragile first-order of approximation. Their common sales distribution produce the same linearization, but their different network structures lead to different nonlinearities. Hence, in the context of aggregate fluctuations, networks are neither a particular case of granularity nor an alternative to it. It is simply that the sales distribution is a sufficient statistic for the network at the first order but not at higher orders.

\section{General Framework}

In this section, we set up a non-parametric general equilibrium model to demonstrate both Hulten's theorem as well as our second-order approximation. Final demand is represented as the maximizer of a constant-returns aggregator of final demand for individual goods

$$
Y=\max _{\left\{c_{1}, \ldots, c_{N}\right\}} \mathcal{D}\left(c_{1}, \ldots, c_{N}\right)
$$

subject to the budget constraint

$$
\sum_{i=1}^{N} p_{i} c_{i}=\sum_{f=1}^{F} w_{f} \bar{l}_{f}+\sum_{i=1}^{N} \pi_{i}
$$

where $c_{i}$ is the representative household's consumption of good $i, p_{i}$ is the price and $\pi_{i}$ is the profit of producer $i, w_{f}$ is the wage of factor $f$ which is in fixed supply $\bar{l}_{f}$. The two sides of the budget constraint coincide wtih nominal GDP, using respectively the final expenditure and income approaches.

Each good $i$ is produced by competitive firms using the production function

$$
y_{i}=A_{i} F_{i}\left(l_{i 1}, \ldots, l_{i F}, x_{i 1}, \ldots, x_{i N}\right)
$$

where $A_{i}$ is Hicks-neutral technology, $x_{i j}$ are intermediate inputs of good $j$ used in the production of good $i$, and $l_{i f}$ is labor type $f$ used by $i$. The profits earned by the producer of good $i$ are

$$
\pi_{i}=p_{i} y_{i}-\sum_{f=1}^{F} w_{f} l_{i f}-\sum_{j=1}^{N} p_{j} x_{i j}
$$

The market clearing conditions for goods $1 \leq i \leq N$ and factors $1 \leq f \leq F$ are

$$
y_{i}=\sum_{j=1}^{N} x_{j i}+c_{i} \text { and } \bar{l}_{f}=\sum_{i=1}^{N} l_{i f} .
$$


Competitive equilibrium is defined in the usual way, where all agents take prices as given, and markets for every good and every type of labor clears.

We interpret $Y$ as a cardinal measure of (real) aggregate output and note that it is the correct measure of the household's "standard of living" in this model. We implicitly rely on the existence of complete financial markets and homotheticity of preferences to ensure the existence of a representative consumer. Although the assumption of a representative consumer is not strictly necessary for the results in this section, it is a standard assumption in this literature since it allows us to unambiguously define and measure changes in real aggregate output without contending with the issue of the appropriate price index.

We assume that the production function $F_{i}$ of each good $i$ has constant returns to scale, which implies that equilibrium profits are zero. This assumption is less restrictive than it may appear because decreasing returns to scale can be captured by adding fixed factors to which the corresponding profits accrue. ${ }^{10}$ A similar observation applies to the assumption that shocks are Hicks-neutral: we can represent a productivity shock augmenting a specific input by adding a new producer that produces this input and hitting this new producer with a Hicks-neutral shock. ${ }^{11}$ Note also that although we refer to each producer as producing one good, our framework actually allows for joint production by multi-product producers: for example, to capture a producer $i$ producing goods $i$ and $i^{\prime}$ using intermediate inputs and factors, we represent good $i^{\prime}$ as an input entering negatively in the production and cost functions for good $i{ }^{12}$ Finally, note that goods could represent different varieties of goods from the same industry, goods from different industries, or even goods in different time periods, regions, or states of nature. ${ }^{13}$

Define $Y\left(A_{1}, \ldots, A_{N}\right)$ to be the equilibrium aggregate output as a function of the exogenous technology levels. Throughout the paper, and without loss of generality, we derive results regarding the effects of shocks in the vicinity of the steady state, which we normalize

\footnotetext{
${ }^{10}$ Our formulas can also in principle be applied with increasing-returns to scale under the joint assumption of marginal-cost pricing and impossibility of shutting down production, by simply adding producer-specific fixed factors with negative marginal products and negative payments (these factors are "bads" that cannot be freely disposed of).

${ }^{11}$ Shocks to the composition of demand can be captured in the same way via a set of consumer-specific productivity shocks. For example, if the final demand aggregator is CES with an elasticity strictly greater than one, an increase in consumer demand for $i$ can be modeled as a positive consumer-specific productivity shock to $i$ and a set of negative consumer-specific productivity shocks to all other final goods such that the consumption-share-weighted sum of the shocks is equal to zero. The sign of the shocks must be reversed if the elasticity of substitution is strictly lower than one, and the Cobb-Douglas case can be treated as a limit. These constructions generalize beyond the CES case. Hulten's theorem implies that shocks to the composition of demand have no first-order effect on aggregate output, but in general, they have nonzero second-order (and more generally nonlinear) effects.

${ }^{12}$ To satisfactorily capture such features, one probably needs to go beyond the nested-CES case of Section 4 and use instead the non-parametric generalization to arbitrary economies provided in Section 5.

${ }^{13}$ If we apply the model to different periods of time and states of nature, then $Y$ corresponds to an intertemporal aggregate consumption index reflecting intertemporal welfare.
} 
to be $\left(A_{1}, \ldots, A_{N}\right)=(1, \ldots, 1)$. All the relevant derivatives are evaluated at that point.

Theorem 1 (Hulten 1978). The first-order macroeconomic impact of microeconomic shocks is given by: ${ }^{14}$

$$
\frac{\mathrm{d} \log Y}{\mathrm{~d} \log A_{i}}=\lambda_{i}
$$

where $\lambda_{i}=p_{i} y_{i} /\left(\sum_{j=1}^{N} p_{j} c_{j}\right)$ the sales of producer $i$ as a fraction of GDP or Domar weight.

Hulten's theorem can be seen as a consequence of the first welfare theorem: since this economy is efficient, $Y\left(A_{1}, \ldots, A_{N}\right)$ is also the social planning optimum and prices are the multipliers on the resource constraints for the different goods. Applying the envelope theorem to the social planning problem delivers the result.

Hulten's theorem has the powerful implication that, to a first-order, the underlying microeconomic details of the structural model are completely irrelevant as long as we observe the equilibrium sales distribution: the shape of the production network, the microeconomic elasticities of substitution in production, the degree of returns to scale, and the extent to which inputs and factors can be reallocated, are all irrelevant.

We now provide a characterization of the second-order effects in terms of reducedform elasticities. We need to introduce two objects: GE elasticities of substitution, and the input-output multiplier. Later on, we show how these reduced-form elasticities arise from structural primitives using a structural model.

We start by introducing the GE elasticities of substitution. Recall that for any homogeneous of degree one function $f\left(A_{1}, \ldots, A_{N}\right)$, the Morishima (1967) elasticity of substitution is

$$
\frac{1}{\rho_{j i}}=\frac{\mathrm{d} \log \left(M R S_{j i}\right)}{\mathrm{d} \log \left(A_{i} / A_{j}\right)}=\frac{\mathrm{d} \log \left(f_{j} / f_{i}\right)}{\mathrm{d} \log \left(A_{i} / A_{j}\right)^{\prime}}
$$

where $M R S_{i j}$ is the ratio of partial derivatives with respect to $A_{i}$ and $A_{j}$, and $f_{i}=\mathrm{d} f / \mathrm{d} A_{i} \cdot{ }^{15}$

When the homothetic function $f$ corresponds to a CES utility function and $A_{i}$ to quantities, $\rho_{i j}$ is the associated elasticity of substitution parameter. However, we do not impose this interpretation, and instead treat this object as a reduced-form measure of the curvature of isoquants. By analogy, we define a pseudo elasticity of substitution for non-homothetic functions in a similar fashion.

\footnotetext{
${ }^{14}$ In the special case where $A_{i}$ is a factor-augmenting shock, the relevant $\lambda_{i}$ corresponds to a producer's bill for this factor as a share of GDP. This is because if we relabel the labor input of producer $i$ as a new producer, we can represent a factor-augmenting shock to $i$ 's labor as a Hicks-neutral shock to this new producer.

${ }^{15}$ This is a generalization of the two-variable elasticity of substitution introduced by Hicks (1932) and analyzed in detail by Blackorby and Russell (1989).
} 
Definition 1. For any smooth function $f: \mathbb{R}^{N} \rightarrow \mathbb{R}$, the pseudo elasticity of substitution is

$$
\frac{1}{\rho_{j i}} \equiv \frac{\mathrm{d} \log \left(M R S_{j i}\right)}{\mathrm{d} \log A_{i}}=\frac{\mathrm{d} \log \left(f_{j} / f_{i}\right)}{\mathrm{d} \log A_{i}} .
$$

The pseudo elasticity of substitution is a generalization of the Moroshima elasticity of substitution in the sense that whenever $f$ is homogenous of degree one, the pseudo elasticity is the same as the Moroshima elasticity of substitution.

When applied to the equilibrium aggregate output function of a general equilibrium economy, we call the pseudo elasticity of substitution the general equilibrium pseudo elasticity of substitution or GE elasticity of substitution for short. The GE elasticity of substitution $\rho_{j i}$ is interesting because it measures changes in the relative sales shares of $j$ and $i$ when there is an exogenous shock to $i$. This follows from the fact that

$$
\frac{\mathrm{d} \log \left(\lambda_{i} / \lambda_{j}\right)}{\mathrm{d} \log A_{i}}=\frac{\mathrm{d} \log \left[\left(Y_{i} A_{i}\right) /\left(Y_{j} A_{j}\right)\right]}{\mathrm{d} \log A_{i}}=1+\frac{\mathrm{d} \log \left(Y_{i} / Y_{j}\right)}{\mathrm{d} \log A_{i}}=1-\frac{1}{\rho_{j i}}
$$

where the first equality applies Hulten's theorem. A decrease in the productivity of $i$ causes $\lambda_{i} / \lambda_{j}$ to increase when $\rho_{j i} \in(0,1)$, and to decrease otherwise. We say that a $j$ is a GEcomplement for $i$ if $\rho_{j i} \in(0,1)$, and a GE-substitute otherwise. When $f$ is a CES aggregator, this coincides with the standard definition of gross complements and substitutes. As usual, when $f$ is Cobb-Douglas, $i$ and $j$ are neither substitutes nor complements. In general, GE-substitutability is not reflexive.

An important special case is when the shock $\mathrm{d} \log A_{f}$ hits the stock of a factor. In that case, Hulten's theorem implies that $\mathrm{d} \log Y / \mathrm{d} \log A_{f}=\Lambda_{f}$, where $\Lambda_{f}=w_{f} \bar{l}_{f} /\left(\sum_{j=1}^{N} p_{j} c_{j}\right)$ is the share of factor $f$ in GDP. Since $\sum_{f=1}^{F} w_{f} \bar{l}_{f}=G D P$, Euler's theorem implies that the aggregate output is homogenous of degree one in the supplies of the factors. This implies that the general equilibrium pseudo elasticity of substitution between two factors can be interpreted as a genuine elasticity of substitution between these factors in general equilibrium. ${ }^{16}$

Next,we introduce the input-output multiplier.

Definition 2. The input-output multiplier is

$$
\xi \equiv \sum_{i=1}^{N} \frac{\mathrm{d} \log Y}{\mathrm{~d} \log A_{i}}=\sum_{i=1}^{N} \lambda_{i}
$$

\footnotetext{
${ }^{16}$ The difference between an elasticity of substitution and a pseudo elasticity of substitution is that the former is the elasticity of the ratio of marginal rates of substitution with respect to the ratio of two arguments, whereas the latter is the elasticity of marginal rates of substitution with respect to an argument. The two definitions are equivalent whenever the function they are applied to is homogeneous of degree one.
} 
When $\xi>1$, total sales of the shocked quantities exceed total income: an indication that there are intermediate inputs. When $\xi>1$, the impact of a uniform technology shock is correspondingly amplified due to the fact that goods are reproducible. The input-output multiplier $\xi$ captures the percentage change in aggregate output in response to a uniform one-percent increase in technology. Loosely speaking, it captures a notion of returns-toscale at the aggregate level. Changes $\mathrm{d} \log \xi / \mathrm{d} \log A_{i}$ in the input-output multiplier can be interpreted as another kind of GE elasticity of substitution: namely the substitution between the underlying factors (whose payments are GDP) and the reproducible goods (whose payments are sales). ${ }^{17}$

Having defined the GE elasticities of substitution and the input-output multiplier, we are in a position to characterize the second-order terms. We start by investigating the impact of an idiosyncratic shock.

\section{Idiosyncratic Shocks}

Theorem 2 (Second-Order Macroeconomic Impact of Microeconomic Shocks). The secondorder macroeconomic impact of microeconomic shocks is given by ${ }^{18}$

$$
\frac{\mathrm{d}^{2} \log Y}{\mathrm{~d} \log A_{i}^{2}}=\frac{\mathrm{d} \lambda_{i}}{\mathrm{~d} \log A_{i}}=\frac{\lambda_{i}}{\xi} \sum_{\substack{1 \leq j \leq N \\ j \neq i}} \lambda_{j}\left(1-\frac{1}{\rho_{j i}}\right)+\lambda_{i} \frac{\mathrm{d} \log \xi}{\mathrm{d} \log A_{i}} .
$$

The second-order impact of a shock to $i$ is equal to the change in $i^{\prime}$ s sales share $\lambda_{i}$. The change in $i$ 's share of sales is the change in the aggregate sales to GDP ratio, minus the change in the share of sales of all other industries. The former is measured by the

\footnotetext{
${ }^{17}$ The input-output multiplier is called the intermediate input multiplier in a stylized model by Jones (2011), but it also appears under other names in many other contexts. It is also related to the network influence measure of Acemoglu et al. (2012), the granular multiplier of Gabaix (2011), the international fragmentation measure of Feenstra and Hanson (1996), the production chain length multiplier in Kim et al. (2013), and even the capital multiplier in the neoclassical growth model since capital can be trated as an intertemporal intermediate input. It also factors into how the introduction of intermediate inputs amplifies the gains from trade in Costinot and Rodriguez-Clare (2014). Although these papers feature multiplier effects due to the presence of round-about production (either via intermediate inputs or capital), they do not take into account the fact that this multiplier effect can respond to shocks. This is either because they assume Cobb-Douglas functional forms or because they focus on first-order effects.

${ }^{18}$ In the case where shocks are factor-augmenting, the aggregate output function is homogeneous of degree 1 and the formula becomes$$
\frac{\mathrm{d}^{2} \log Y}{\mathrm{~d} \log A_{i f}^{2}}=\lambda_{i f} \sum_{\substack{1 \leq j \leq N \\ 1 \leq g \leq F \\(j, g) \neq(i, f)}} \lambda_{j g}\left(1-\frac{1}{\rho_{j g, i f}}\right),
$$

where $A_{i f}$ is a shock augmenting factor $f$ in the production of good $i, \lambda_{i f}$ is the share of factor $f$ in GDP arising from its use by producer $i$, and $\rho_{j g, i f}$ is the GE elasticity of substitution between factor $f$ in the production of good $i$ and factor $g$ in the production of good $j$.
} 
elasticity of the input-output multiplier $\xi$, while the latter depends on the GE elasticities of substitution. Collectively, the sales shares $\lambda_{i}$, the reduced-form elasticities $\rho_{j i}$, and the reduced-form elasticity of the input-output multiplier $\mathrm{d} \log \xi / \mathrm{d} \log A_{i}$ are sufficient statistics for the response of how aggregate output to to productivity shocks up to a second order.

This result implies that Hulten's first-order approximation is globally accurate if reducedform elasticities are unitary $\rho_{j i}=1$ for every $j$ and if the input-output multiplier $\xi$ is independent of the shock $A_{i}$. We shall see that this amounts to assuming Cobb-Douglas production and consumption functions where sales shares and more generally the whole input-output matrix are constant. The model is then log-linear.

Outside of this special case, there are nonlinearities, and the quality of the first-order approximation deteriorates as the shocks become bigger. The deterioration can be extreme, with the aggregate output function becoming nearly non-smooth, when $\rho_{j i}$ approaches 0 for any $j$, either from above or from below. As we shall see, these arise in the cases of extreme microeconomic complementarities with no reallocation or extreme microeconomic substitutabilities with full reallocation. In these limiting cases, the first-order approximation is completely uninformative, even for arbitrarily small shocks. Similar observations apply when $\mathrm{d} \log \xi / \mathrm{d} \log A_{i}$ approaches infinity. Therefore, although the Cobb-Douglas special case is very popular in the literature, it constitutes a very special case where the second-order terms are all identically zero. ${ }^{19}$

The second-order approximation

$$
\log Y \approx \log \bar{Y}+\frac{\mathrm{d} \log Y}{\mathrm{~d} \log A_{i}} \log A_{i}+\frac{1}{2} \frac{\mathrm{d}^{2} \log Y}{\mathrm{~d} \log A_{i}^{2}}\left(\log A_{i}\right)^{2}
$$

of the aggregate output function with respect to the productivity of producer $i$ can then be written as

$$
\log Y \approx \log \bar{Y}+\lambda_{i} \log A_{i}+\frac{1}{2} \frac{\lambda_{i}}{\xi} \sum_{\substack{1 \leq j \leq N \\ j \neq i}} \lambda_{j}\left(1-\frac{1}{\rho_{j i}}\right)\left(\log A_{i}\right)^{2}+\frac{1}{2} \lambda_{i} \frac{\mathrm{d} \log \xi}{\mathrm{d} \log A_{i}}\left(\log A_{i}\right)^{2},
$$

where $\bar{Y}$ is $Y$ evaluated at the steady-state technology values. When goods are GEcomplements, the second-order terms amplify the effect of negative shocks and attenuate the effect of positive shocks relative to the first-order approximation. Instead when goods are GE-substitutes, the second-order approximation attenuates the negative shocks and amplifies the positive shocks instead. A similar intuition holds for the input-output multiplier: if the input-output multiplier is increasing, then the second-order approximation amplifies

\footnotetext{
${ }^{19}$ See for example Acemoglu et al. (2012), Long and Plosser (1983), Bigio and La'O (2016), Acemoglu et al. (2017), Bartelme and Gorodnichenko (2015).
} 
positive shocks and dampens negative shocks, and if this multiplier is decreasing, then the opposite is true.

\section{Correlated Shocks}

To compute the second-order approximation

$\log Y \approx \log \bar{Y}+\sum_{1 \leq i \leq N} \frac{\mathrm{d} \log Y}{\mathrm{~d} \log A_{i}} \log A_{i}+\sum_{1 \leq i \leq N} \frac{1}{2} \frac{\mathrm{d}^{2} \log Y}{\mathrm{~d} \log A_{i}^{2}}\left(\log A_{i}\right)^{2}+\frac{1}{2} \sum_{\substack{1 \leq i \leq N \\ 1 \leq \leq \leq N \\ i \neq j}} \frac{\mathrm{d}^{2} \log Y}{\mathrm{~d} \log A_{j} \mathrm{~d} \log A_{i}} \log A_{i} \log A_{j}$

of the aggregate production function with respect to to shocks to several producers at once we must extend these results to cover the off-diagonal terms in its the Hessian.

Proposition 3 (Correlated Shocks). The second-order macroeconomic impact of correlated microeconomic shocks is given by

$$
\frac{\mathrm{d}^{2} \log Y}{\mathrm{~d} \log A_{j} \mathrm{~d} \log A_{i}}=\frac{\mathrm{d} \lambda_{i}}{\mathrm{~d} \log A_{j}}=\frac{\lambda_{i}}{\xi} \sum_{\substack{1 \leq k \leq N \\ k \neq j}} \lambda_{k}\left(1-\frac{1}{\rho_{k j}}\right)+\lambda_{i} \frac{\mathrm{d} \log \xi}{\mathrm{d} \log A_{j}}-\lambda_{i}\left(1-\frac{1}{\rho_{i j}}\right) . \quad(i \neq j)
$$

The second-order effect of a common shock to $i$ and $j$ is not simply the sum of the secondorder impacts of the idiosyncratic shocks to $i$ and to $j$, and instead there are interactions between the two shocks. ${ }^{20}$ In Section 4 , we provide an explicit characterization of the Hessian in terms of microeconomic primitives.

\section{Macro Moments}

We can use the second-order terms to approximate an economy's macroeconomic moments. To illustrate this intuition while preserving expositional simplicity, we consider shocks to a single producer $i$ which are lognormal with mean $\log 0$ and variance $\sigma^{2} .{ }^{21}$

We first consider average log aggregate output $\mu_{Y}$, for which a Taylor approximation

\footnotetext{
${ }^{20}$ We can also use these ideas to capture the impact of an aggregate shock to the economy, since an aggregate shock is simply a common shock that affects all industries. If $A$ is an aggregate productivity shock, then $\frac{\mathrm{d}^{2} \log Y}{\mathrm{~d} \log A^{2}}=\xi \sum_{i=1}^{N} \frac{\mathrm{d} \log \xi}{\mathrm{d} \log A_{i}}$. So, for aggregate shocks, deviations from Hulten's theorem can only come from the input-output multiplier.

${ }^{21}$ In Appendix E, we include the approximation equations for mean, variance, and skewness for multivariate shocks.
} 
yields

$$
\mu_{Y}=E(\log (Y / \bar{Y})) \approx \frac{1}{2} \frac{\mathrm{d}^{2} \log Y}{\mathrm{~d} \log A_{i}^{2}} \sigma^{2}=\frac{1}{2}\left(\frac{\lambda_{i}}{\xi} \sum_{\substack{1 \leq j \leq N \\ j \neq i}} \lambda_{j}\left(1-\frac{1}{\rho_{j i}}\right)+\lambda_{i} \frac{\mathrm{d} \log \xi}{\mathrm{d} \log A_{i}}\right) \sigma^{2} .
$$

Average log aggregate output can be nonzero even though the technology shocks have zero log average, and it has the same sign as the second-order term. For example, when the second-order terms is negative, corresponding to GE-complementarities, average log aggregate output is lower than its deterministic steady state because nonlinearities magnify negative shocks and attenuate positive shocks.

Second-order terms also shape higher moments of the distribution of aggregate output. The variance is given by

$$
\sigma_{Y}^{2}=\operatorname{Var}(\log (Y / \bar{Y})) \approx\left(\lambda_{i}^{2}+2\left(\frac{\mu_{Y}}{\sigma}\right)^{2}\right) \sigma^{2} \geq \lambda_{i}^{2} \sigma^{2},
$$

where the right-hand side is the variance of the log-linear approximation. This shows that nonlinearities tend to increase the implied variance of aggregate output.

Similarly, the skewness is given by

$$
E\left(\left(\frac{\log (Y / \bar{Y})-\mu_{Y}}{\sigma_{Y}}\right)^{3}\right) \approx \frac{2 \mu_{Y}}{\sigma_{Y}^{3}}\left(4 \mu_{Y}+3 \lambda_{i}^{2} \sigma^{2}\right) .
$$

For example, when the second-order term is negative, the distribution of aggregate output is skewed to the left since nonlinearities magnify negative shocks and attenuate positive shocks, even though the technology shocks are symmetric. Typical negative deviations of aggregated output are then larger than typical positive deviations.

Finally, the kurtosis is given by

$$
E\left(\left(\frac{\log (Y / \bar{Y})-\mu_{Y}}{\sigma_{Y}}\right)^{4}\right) \approx 3\left(1+\left(\frac{\mu_{Y}}{\sigma}\right)^{2} \frac{22\left(\mu_{Y} / \sigma\right)^{2}+7 \lambda_{i}^{2}}{\left(\lambda_{i}^{2}+2\left(\mu_{Y} / \sigma\right)^{2}\right)^{2}}\right) \geq 3 .
$$

Aggregate output has excess kurtosis when second-order terms are nonzero. For example, when the second-order term is negative, the left tail is fattened because negative shocks are magnified, and this gives rise to excess kurtosis, even though the technology shocks are symmetric and thin-tailed. A higher share of the variance is then due to negative, infrequent, extreme deviations, as opposed to symmetric, frequent, and modestly sized deviations. 
The importance of all these effects increases with the variance $\sigma^{2}$ of the shocks because they are driven by nonlinearities, and because the importance of nonlinearities increases with the size of the shocks.

\section{Welfare Costs of Sectoral Shocks}

For the majority of the paper, we focus on log aggregate output, which can be characterized with unitless elasticities. With complementarities, we have argued that sector shocks lower the mean of log aggregate output, an effect which we can interpret as the welfare cost of sectoral shocks. One may imagine that the losses from uncertainty that we identify depend on the concavity of the $\log$ function. A consumer with log utility prefers a mean-preserving reduction in uncertainty even when the aggregate output function is linear. However, as shown by Lucas (1987), the corresponding losses are extremely small in practice in business-cycle settings. The much larger effects that we identify originate in nonlinearities in production, and they are present even when the utility function is linear in aggregate consumption.

The following proposition formalizes this intuition and shows that the Lucas-style welfare losses due to nonlinearities in the utility function in the form of risk-aversion and the losses due to nonlinearities in production do not interact with one-another up to a secondorder approximation. ${ }^{22}$

Proposition 4 (Welfare Cost of Sectoral Shocks). Let $u: \mathbb{R} \rightarrow \mathbb{R}$ be a utility function and let $Y: \mathbb{R}^{N} \rightarrow \mathbb{R}$ be the aggregate output function. Suppose that productivty shocks have mean 1 and $a$ diagonal covariance matrix with $k$ th diagonal element $\sigma_{k}^{2}$. Then

$$
\frac{u^{\prime}(\bar{Y})}{\bar{Y}}(E(u(Y))-u(\bar{Y})) \approx-\frac{\bar{Y}}{2}\left(\gamma \sum_{k=1}^{N} \lambda_{k}^{2} \sigma_{k}^{2}+\sum_{k=1}^{N} \frac{\mathrm{d}^{2} Y}{\mathrm{~d} A_{k}^{2}} \sigma_{k}^{2}\right),
$$

where $\gamma$ is the coefficient of relative risk aversion at the deterministic steady-state $\bar{Y}$.

The first term on the right-hand side, which is quantitatively small, is the traditional Lucas-style cost arising from curvature in the utility function. The second term, which is quantitatively large, is due to the curvature inherent in production and does not depend on the coefficient of relative risk aversion. ${ }^{23}$

\footnotetext{
${ }^{22}$ In fact, it could easily be the case that a risk-averse household prefers the economy to be subject to stochastic shocks if the economy features macro-substitutability and the second-order terms are positive, which happens in the presence of GE-substitutability.

${ }^{23}$ Proposition 4 is stated idiosyncratic shocks for expositional clarity. In Appendix A, we prove the result for more general utility functions and shocks. For our theoretical results, we find it convenient work with elasticities $\mathrm{d}^{2} \log Y / \mathrm{d} \log A_{k^{\prime}}^{2}$ but we can use these results to compute the welfare cost in Proposition 4 by noting
} 


\section{Mapping From Micro to Macro}

Theorem 2 implies that the GE elasticities of substitution $\rho_{i j}$ and the elasticity of the inputoutput multiplier $\mathrm{d} \log \xi / \mathrm{d} \log A_{i}$ are sufficient statistics for the second-order impact of shocks. However, these sufficient statistics are reduced-form elasticities, and unlike $\lambda_{i}$ and $\xi$, they are not readily observable. Furthermore, since they are general equilibrium objects, they cannot be identified through exogenous microeconomic variation. So, while careful empirical work can identify micro-elasticities, the leap from micro-estimates to macro-effects can be hazardous.

In this paper, we provide the mapping from structural micro parameters to the reducedform GE elasticities. This general characterization can be found in Section 4 for general nested-CES economies, and in Section 5 for arbitrary economies. However, rather than stating these results up front, we build up to the general characterization using some important special cases in Section 3.

\section{Illustrative Examples}

In this section, we work through two special cases to illustrate and isolate some intuition for how the GE elasticities of substitution and the input-output multiplier affect the shape of the aggregate output function. After working through these examples, we provide a generic characterization of the second-order terms in Section 4 and 5.

\subsection{GE Elasticities of Substitution}

To start with, we focus on the GE elasticities of substitution by considering a simple example of a horizontal economy with no intermediate inputs. The input-output multiplier is constant and equal to one, and so deviations from Hulten's theorem are only due to non-unitary GE elasticities of substitution. We emphasize how the GE elasticities of substitution depend not only on the micro elasticities of substitution, but also on the degree to which labor can be reallocated across uses and on the returns to scale in production. Throughout all the upcoming examples, variables with overlines denote steady-state values.

There are $N$ goods produced using the production functions

$$
\frac{y_{i}}{\bar{y}_{i}}=A_{i}\left(\frac{l_{i s_{i}}}{\bar{l}_{i s_{i}}}\right)^{1-\omega_{g}}\left(\frac{l_{i g}}{\bar{l}_{i g}}\right)^{\omega_{g}},
$$

that $\mathrm{d}^{2} Y / \mathrm{d} A_{k}^{2}=\bar{Y} \mathrm{~d}^{2} \log Y / \mathrm{d} \log A_{k}^{2}-\bar{Y} \lambda_{k}\left(1-\lambda_{k}\right)$. 
where $l_{i s_{i}}$ and $l_{i g}$ are the amounts of the specific and general labor used by producer $i$. The specific labor of type $i$ can only be used by producer $i$ and the general labor can be used by all producers.

The household's consumption function is

$$
\frac{Y}{\bar{Y}}=\left(\sum_{i=1}^{N} \omega_{0 i}\left(\frac{c_{i}}{\bar{c}_{i}}\right)^{\frac{\theta_{0}-1}{\theta_{0}}}\right)^{\frac{\theta_{0}}{\theta_{0}-1}}
$$

where $\theta_{0}$ is the microeconomic elasticity of substitution in consumption and $\sum_{i=1}^{N} \omega_{0 i}=1$.

The specific labors and the general labor are in fixed supplies at $\bar{l}_{s_{i}}=\bar{l}_{i s_{i}}$ and $\bar{l}_{g}=\sum_{i=1}^{N} \bar{l}_{i g}$. The market-clearing conditions are

$$
c_{i}=y_{i}, \quad \bar{l}_{s_{i}}=l_{i s_{i}}, \quad \text { and } \quad \bar{l}_{g}=\sum_{i=1}^{N} l_{i g} .
$$

Different degrees of labor reallocation can be expected depending on the degree of aggregation. The the time horizon is also important since we might expect labor to be more difficult to adjust at short horizons than at long horizons. Some of these dynamic effects can be captured by performing comparative statics with respect to $\omega_{g}$, where $\omega_{g}=0$ represents an economy where labor cannot be reallocated, and $\omega_{g}=1$ an economy where labor can be fully reallocated.

Proposition 5. In the horizontal economy, the sales shares are given by $\lambda_{i}=\omega_{0 i}$, the input-output multiplier is constant with $\xi=1$ and $\mathrm{d} \log \xi / \mathrm{d} \log A_{i}=0$. The GE elasticities of substitution are all equal and are given by

$$
\rho_{j i}=\rho=\frac{\theta_{0}\left(1-\omega_{g}\right)+\omega_{g}}{\theta_{0}\left(1-\omega_{g}\right)+\omega_{g}+\left(1-\theta_{0}\right)} .
$$

The second-order macroeconomic impact of microeconomic shocks is given by

$$
\frac{\mathrm{d}^{2} \log Y}{\mathrm{~d} \log A_{i}^{2}}=\frac{\mathrm{d} \lambda_{i}}{\mathrm{~d} \log A_{i}}=\lambda_{i}\left(1-\lambda_{i}\right)\left(1-\frac{1}{\rho}\right)
$$

To build intuition, we consider the polar cases with no-reallocation where $\omega_{g}=0$ and with full reallocation where $\omega_{g}=1$. We start with the no-reallocation case where $\omega_{g}=0$. Because labor cannot be moved across producers, it is as if there were a fixed endowment 
of each good and so aggregate output is given by:

$$
\frac{Y}{\bar{Y}}=\left(\sum_{i=1}^{N} \omega_{0 i} A_{i}^{\frac{\theta_{0}-1}{\theta_{0}}}\right)^{\frac{\theta_{0}}{\theta_{0}-1}} .
$$

The GE elasticities of substitution and the microeconomic elasticities of substitution coincide so that $\rho=\theta_{0}$. Since $\theta_{0} \in[0, \infty)$ we have $\rho \in[0, \infty)$. The second-order macroeconomic impact of microeconomic shocks is given by

$$
\frac{\mathrm{d}^{2} \log Y}{\mathrm{~d} \log A_{i}^{2}}=\frac{\mathrm{d} \lambda_{i}}{\mathrm{~d} \log A_{i}}=\lambda_{i}\left(1-\lambda_{i}\right)\left(1-\frac{1}{\theta_{0}}\right) .
$$

In the Cobb-Douglas case $\theta_{0}=1$, second-order terms are identically equal to zero and the first-order approximation of Hulten's theorem is globally accurate. The quality of the first-order approximation deteriorates as we move away from $\theta_{0}=1$ in both directions. The second-order term is negative when $\theta_{0}<1$ and positive when $\theta_{0}>1$. Relative to the first-order approximation, the second-order approximation amplifies negative shocks and mitigates positive shocks in the former case and the reverse the latter case.

To build intuition, it is useful to inspect how the relative sales share $\lambda_{i} / \lambda_{j}$ of $i$ versus $j$ changes in response to a shock to $i$ :

$$
\frac{\mathrm{d} \log \left(\lambda_{i} / \lambda_{j}\right)}{\mathrm{d} \log A_{i}}=\frac{\mathrm{d} \log \left(p_{i} / p_{j}\right)}{\mathrm{d} \log A_{i}}+\frac{\mathrm{d} \log \left(y_{i} / y_{j}\right)}{\mathrm{d} \log A_{i}}=\frac{\mathrm{d} \log \left(p_{i} / p_{j}\right)}{\mathrm{d} \log A_{i}}+\frac{\mathrm{d} \log \left(A_{i} / A_{j}\right)}{\mathrm{d} \log A_{i}}=1-\frac{1}{\theta_{0}} .
$$

Because there is no reallocation, the relative quantity $y_{i} / y_{j}$ moves one-for-one with the shock to $i$. In the Cobb-Douglas case $\theta_{0}=1$, the relative price $p_{i} / p_{j}$ moves one-for-one in the opposite direction, and so the relative share $\lambda_{i} / \lambda_{j}$ remains constant. When $\theta_{0}<1$, the relative price moves more than one-for-one with the shock, and so the relative share increases when the shock is negative, and increases when it is positive. When $\theta_{0}>1$, the relative price moves less than one-for-one with the shock, and so the relative share decreases when the shock is negative, and increases when it is positive.

Consider the Leontief limit $\theta_{0} \rightarrow 0$. In this limit, deviations from the first-order approximation become so large that the first-order term becomes completely uninformative. Following a negative shock to $i$, the relative price $p_{i} / p_{j}$ jumps to infinity, and so does the relative share $\lambda_{i} / \lambda_{j}$. Following a positive shock, the relative price jumps to zero and so does the relative share. The associated amplification of negative shocks and mitigation of negative shocks is extreme.

Let us now consider the perfect-substitutes limit $\theta_{0} \rightarrow \infty$. Positive shocks are amplified 


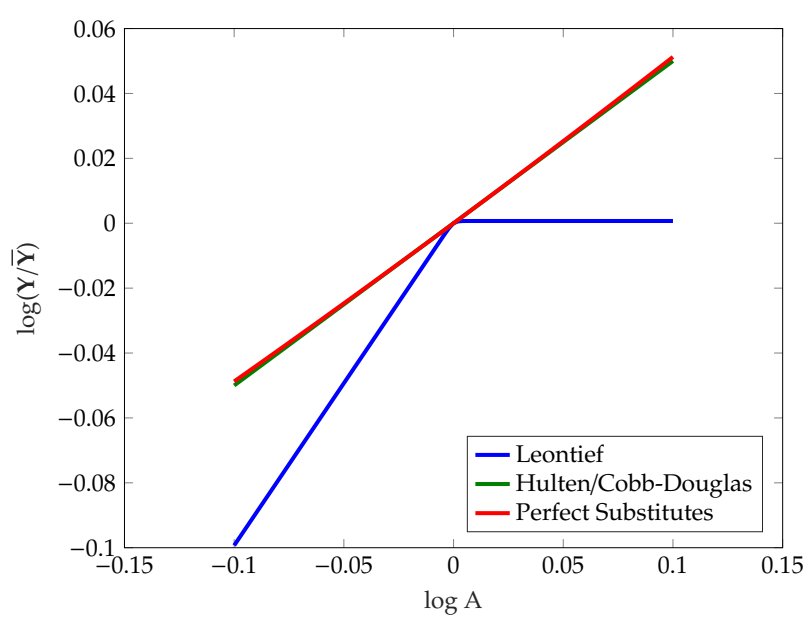

(a) log aggregate output with no reallocation/extreme decreasing returns. Perfect substitutes and Hulten's approximation overlap almost perfectly.

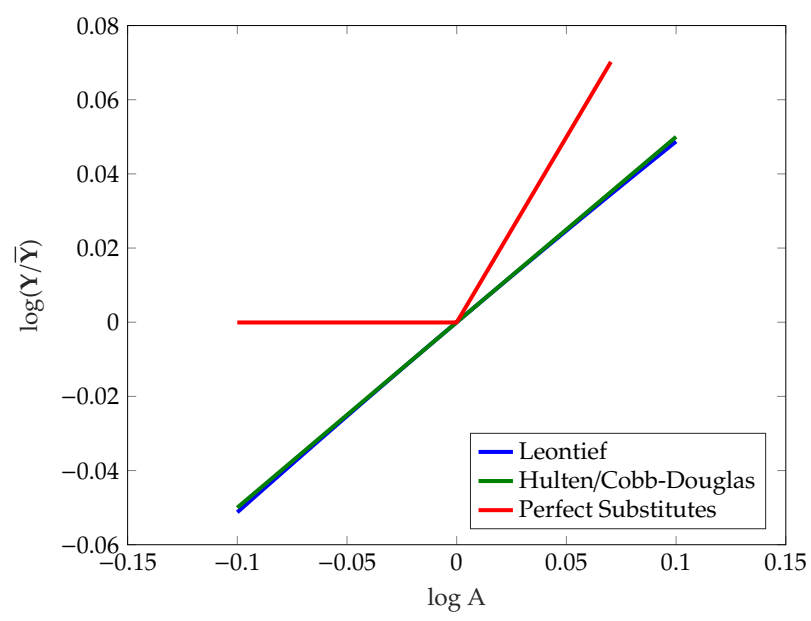

(b) log aggregate output with full reallocation/constant returns. Leontief and Hulten's approximation overlap almost perfectly.

Figure 1: $\log$ aggregate output as a function of productivity $\log A_{i}$ in the economy with full reallocation/constant returns for different values of $\theta_{0}$. This example consists of two, equally, sized industries using labor as their only input. The economies depicted in Figures $1 \mathrm{a}$ and $1 \mathrm{~b}$ are all equivalent to a first-order.

and negative shocks are mitigated, but the effect is not nearly so dramatic. In fact, because goods are perfect substitutes, the relative price $p_{i} / p_{j}$ is constant. Therefore, the relative share $\lambda_{i} / \lambda_{j}$ moves one-for-one with the shock to $i$. The situation is depicted graphically in Figure 1a.

Having analyzed the case with no labor reallocation, we now consider the polar opposite case, where labor can be costlessly reallocated across producers and can be used with constant returns to scale so that $\omega_{g}=1$. Solving out the allocation of labor to each producer and replacing leads to the following expression for aggregate output:

$$
\frac{Y}{\bar{Y}}=\frac{\left(\sum_{i=1}^{N} \omega_{0 i} A_{i}^{\theta_{0}-1}\right)^{\frac{\theta_{0}}{\theta_{0}-1}}}{\sum_{i=1}^{N} \omega_{0 i} A_{i}^{\theta_{0}-1}} .
$$

In this case, the GE elasticities of substitution do not typically coincide with the structural microeconomic elasticity of substitution since we have $\rho=1 /\left(2-\theta_{0}\right)$. Because $\theta_{0} \in[0, \infty)$, we have $\rho \in(-\infty, 0) \cup[1 / 2,+\infty)$. The second-order macroeconomic impact of microeconomic shocks is given by

$$
\frac{\mathrm{d}^{2} \log Y}{\mathrm{~d} \log A_{i}^{2}}=\frac{\mathrm{d} \lambda_{i}}{\mathrm{~d} \log A_{i}}=\lambda_{i}\left(1-\lambda_{i}\right)\left(\theta_{0}-1\right) .
$$

As above, in the Cobb-Douglas case $\theta_{0}=1$, second-order terms are identically equal to 
zero and the first-order approximation of Hulten's theorem is globally accurate. The secondorder term is negative when $\theta_{0}<1$ and positive when $\theta_{0}>1$. Relative to the first-order approximation, the second-order approximation amplifies negative shocks and mitigates positive shocks in the former case and the reverse the latter case. However, this time, the second-order term becomes singular when the goods are highly substitutable rather than when they are highly complementary.

Once again, we can unpack this result by noting that

$$
\frac{\mathrm{d} \log \left(\lambda_{i} / \lambda_{j}\right)}{\mathrm{d} \log A_{i}}=\frac{\mathrm{d} \log \left(p_{i} / p_{j}\right)}{\mathrm{d} \log A_{i}}+\frac{\mathrm{d} \log \left(y_{i} / y_{j}\right)}{\mathrm{d} \log A_{i}}=\frac{\mathrm{d} \log \left(A_{j} / A_{i}\right)}{\mathrm{d} \log A_{i}}+\frac{\mathrm{d} \log \left(y_{i} / y_{j}\right)}{\mathrm{d} \log A_{i}}=\theta_{0}-1
$$

Because labor can be costlessly reallocated across producers, the relative price $p_{i} / p_{j}$ always moves inversely one-for-one with the shock to $i$. In the Cobb-Douglas case, the relative quantity $y_{i} / y_{j}$ moves one for one with the shock to $i$, and the relative share $\lambda_{i} / \lambda_{j}$ remains constant. When $\theta_{0}<1$, the relative quantity moves less than one-for-one with the shock as labor is reallocated towards $i$ if the shock is negative and away from $i$ if the shock is positive. As a result, the relative share increases when the shock is negative, and increases when it is positive. When $\theta_{0}>1$, relative quantity moves more than one-for-one with the shock as labor is reallocated away from $i$ when the shock is negative and towards $i$ when it is positive. As a result, the relative share decreases when the shock is negative, and increases when it is positive.

Contrary to what one may have assumed, a near-Leontief production function is not sufficient for generating large deviations from Hulten's theorem, as long as factors can be reallocated freely, precisely because this reallocation is successful at reinforcing "weak links". In the Leontief limit, the relative quantity $y_{i} / y_{j}$ is invariant to the shock, and so the relative sales share $\lambda_{i} / \lambda_{j}$ moves inversely one-for-one with the shock to $i$. Relative to the first-order approximation, the second-order approximation still amplifies negative shocks and mitigates positive shocks, but the corresponding magnitudes are much smaller than in the case where labor cannot not be reallocated.

In the perfect-substitutes limit, labor is entirely allocated to the most productive producer. In response to a positive shock to $i$, the relative quantity $y_{i} / y_{j}$ jumps to infinity, and so does the relative share $\lambda_{i} / \lambda_{j}$. In response to a negative shock, the relative quantity drops to zero, and so does the relative share. Relative to the first-order approximation, the secondorder approximation still amplifies positive shocks and mitigates negative shocks, but the corresponding magnitudes are now much larger than in the case where labor cannot not be reallocated. The situation is depicted graphically in Figure 1b.

Finally, note that both when labor can or cannot be reallocated, the second-order term scales in $\lambda_{i}\left(1-\lambda_{i}\right)$ as a function of the size $\lambda_{i}$ of the shocked producer $i$. Its absolute value is 
therefore hump-shaped in $\lambda_{i}$ : it goes zero when $\lambda_{i}$ is close to 0 or 1 , and reaches a maximum when $\lambda_{i}$ is intermediate at $1 / 2$. That the term is small when $\lambda_{i}$ is close to 0 is intuitive. That it is small when $\lambda_{i}$ is close to 1 makes sense since the then economy behaves much like producer $i$ and aggregate output is then close to being proportional to $A_{i}$. The second-order term can only be significant for intermediate values of $\lambda_{i}$.

To recap, with complementarities: a negative shock can cause a large downturn when labor cannot be freely re-allocated, but the ability to re-allocate labor largely mitigates these effects; positive shocks have a lesser impact. By contrast, with substitutabilities: a positive shock can cause a big boom when labor can be re-allocated, but the inability to re-allocated labor mitigates these effects; negative shocks have a lesser impact. Cobb-Douglas stands as a special case where the macroeconomic impact of microeconomic shocks is symmetric independently of whether or not labor can be reallocated (since the equilibrium allocation of labor across producers is constant even when labor can be re-allocated). ${ }^{24}$ These effects are less pronounced when the size of the shocked producer is very small or very large, and are more pronounced when it is intermediate.

\subsection{Input-Output Multiplier}

In the previous example of a horizontal economy, the input-output multiplier $\xi$ is constant and deviations from Hulten's theorem are due to non-unitary GE elasticities of substitution. We now focus on a different example, that of a roundabout economy, where deviations from Hulten's theorem are driven purely by variability in $\xi$, and the GE elasticities of substitution play no role.

The economy has a single good and single factor. Gross output is given by

$$
\frac{y_{1}}{\bar{y}_{1}}=A_{1}\left(\omega_{1 l}\left(\frac{l_{1}}{\bar{l}_{1}}\right)^{\frac{\theta_{1}-1}{\theta_{1}}}+\left(1-\omega_{1 l}\right)\left(\frac{x_{1}}{\bar{x}_{1}}\right)^{\frac{\theta_{1}-1}{\theta_{1}}}\right)^{\frac{\theta_{1}}{\theta_{1}-1}}
$$

where $x_{1}$ is the amount of good 1 used as an intermediate input. The supply of the factor is inelastic at $\bar{l}=\bar{l}_{1}$. Final output $Y=c_{1}$ is produced one-to-one from good 1 .

The market-clearing conditions are

$$
y_{1}=c_{1}+x_{1} \text { and } \quad \bar{l}=l_{1} \text {. }
$$

\footnotetext{
${ }^{24}$ These results are closely related to the findings in Jones (2011), who noted that the relevant CES parameter used in aggregating microeconomic productivity shocks depends on whether or not factors are allocated through the market or assigned exogenously.
} 
The steady-state input-output multiplier

$$
\xi=1+\left(1-\omega_{1 l}\right)+\left(1-\omega_{1 l}\right)^{2}+\ldots=1 / \omega_{1 l}
$$

decreases with the labor share $\omega_{1 l}$ and increases with the intermediate input share $1-\omega_{11}$. Hulten's theorem implies that

$$
\frac{d \log Y}{d \log A_{1}}=\xi
$$

so that the first-order impact of the shock increases with the steady-state input-output multiplier $\xi$.

Proposition 6 (Variable IO multiplier). In the roundabout economy, the input-output multiplier is given by $\xi=1 / \omega_{1 l}$ and its elasticity is given by ${ }^{25}$

$$
\frac{\mathrm{d} \log \xi}{\mathrm{d} \log A_{1}}=(\xi-1)\left(\theta_{1}-1\right)
$$

The second-order macroeconomic impact of microeconomic shocks is given by

$$
\frac{\mathrm{d}^{2} \log Y}{\mathrm{~d} \log A_{1}^{2}}=\frac{\mathrm{d} \xi}{\mathrm{d} \log A_{1}}=\xi(\xi-1)\left(\theta_{1}-1\right)
$$

Hulten's approximation is exact only when there are no intermediate inputs so that $\xi=1$ or when the economy is Cobb-Douglas so that $\theta_{1}=1$. Otherwise, the second-order term is increasing in $\theta_{1}-1$ and in a network term $\xi(\xi-1){ }^{26}$

Intuitively, this results from the fact that output is used as its own input. When $\theta_{1}=1$, the input-output multiplier remains constant. When $\theta_{1}<1$, the input-output multiplier increases if the shock is negative, and decreases if it is positive. When $\theta_{1}>1$, the inputoutput multiplier decreases if the shock is negative, and increases if it is positive. The larger is the steady-state input-output multiplier, the larger is the effect.

Figure 2 plots $\log Y$ as a function of $\log A_{1}$ for the case where $\theta_{1} \approx 0, \theta_{1}=1$, and $\theta_{1}=2$. In the limit $\theta_{1} \rightarrow 0$, output is linear in productivity (rather than loglinear) with slope $1 / \omega_{11}$. When $\theta_{1}=2$, output is hyperbolic in productivity. ${ }^{27}$

\footnotetext{
${ }^{25}$ Proposition 6 shows that even though the gross production function is homogenous in productivity, aggregate net output (value added) is not homogeneous of degree 1. Furthermore, aggregate output is not homogenous of any degree in equilibrium, since $\xi$ varies in response to the shock.

${ }^{26}$ There is no closed-form solution for equilibrium aggregate output in this case.

${ }^{27}$ In this example, when $\theta_{1}=0$, we have $Y=A / \omega_{1 l}$, where $1 / \omega_{1 l}$ is the steady-state input-output multiplier. Therefore, although Hulten's approximation fails in log terms, Hulten's theorem is globally accurate in linear terms. This is a consequence of the fact that there is only one good. In Appendix B, we generalize this example to multiple goods, and show that output can be very strongly nonlinear even with full labor reallocation.
} 


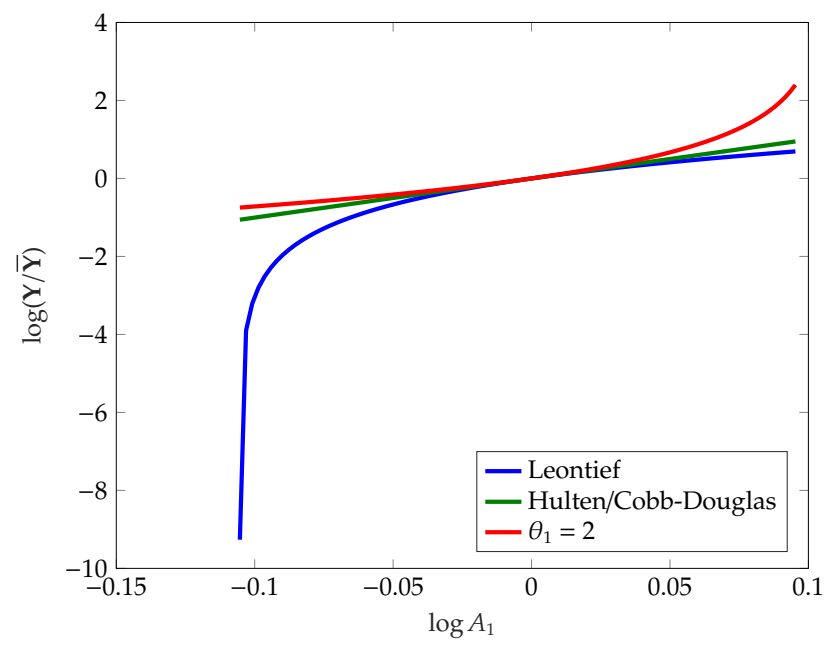

Figure 2: Output as a function of productivity shocks $\log A_{1}$ with variable input-output multiplier effect with steady-state input-output multiplier $\xi=10$.

\section{General Nested-CES Networks}

We now characterize the second-order terms for a general nested-CES economy (encompassing the examples in Section 3). Throughout this section, variables with over-lines are normalizing constants equal to the values in steady-state. ${ }^{28}$

Any nested-CES economy with a representative consumer, an arbitrary numbers of nests, elasticities, and intermediate input use, can be re-written in what we call standard form, which simply means that each CES aggregator corresponds to a node in the production network with a one node-specific elasticity of substitution. Through a relabelling, this structure can represent any nested-CES economy with an arbitrary pattern of nests and elasticities. Intuitively, by relabelling each CES aggregator to be a new producer, we can have as many nests as desired.

Formally, a nested-CES economy in standard form is defined by a tuple $(\omega, \theta, F)$ and a set of normalizing constants $(\bar{y}, \bar{x})$. The $(N+1+F) \times(N+1+F)$ matrix $\omega$ is a matrix of input-output parameters where the first row and column correspond to household sector, the next $N$ rows and columns correspond to reproducible goods and the last $F$ rows and columns correspond to factors. What distinguishes factors from goods is that factors cannot be produced. The $(N+1) \times 1$ vector $\theta$ is a vector of microeconomic elasticities of substitution. For convenience we use number indices starting at 0 instead of 1 to describe the elements of $\omega$ and $\theta .^{29}$

\footnotetext{
${ }^{28}$ Since we are interested in log changes, the normalizing constants are irrelevant. We use normalized quantities since it simplifies calibration, and clarifies the fact that CES aggregators are not unit-less.

${ }^{29}$ We impose the restriction that $\omega_{i j} \in[0,1], \sum_{j=1}^{N+F} \omega_{i j}=1$ for all $0 \leq i \leq N, \omega_{f j}=0$ for all $N+1 \leq f \leq N+F$, $\omega_{0 f}=0$ for all $N+1 \leq f \leq N+F$, and $\omega_{i 0}=0$ for all $0 \leq i \leq N$.
} 
The $F$ factors are modeled as non-reproducible goods and the production function of these goods are endowments

$$
\frac{y_{f}}{\bar{y}_{f}}=1 \text {. }
$$

The other $N+1$ other goods are reproducible with production functions

$$
\frac{y_{i}}{\bar{y}_{i}}=A_{i}\left(\sum_{j=1}^{N+F} \omega_{i j}\left(\frac{x_{i j}}{\bar{x}_{i j}}\right)^{\frac{\theta_{i}-1}{\theta_{i}}}\right)^{\frac{\theta_{i}}{\theta_{i}-1}},
$$

where $x_{i j}$ are intermediate inputs from $j$ used by $i$. Producer 0 represents final-demand and its production function is the final-demand aggregator so that

$$
\frac{Y}{\bar{Y}}=\frac{y_{0}}{\bar{y}_{0}}
$$

where $Y$ is aggregate output and $y_{0}$ is the final good.

The market-clearing conditions for goods and factors $0 \leq i \leq N+F$ are

$$
y_{i}=\sum_{j=0}^{N} x_{j i}
$$

To state our results, we need the following definitions.

Definition 3. The $(N+1+F) \times(N+1+F)$ input-output matrix $\Omega$ is the matrix whose $i j$ th element is equal to the steady-state value of

$$
\Omega_{i j}=\frac{p_{j} x_{i j}}{p_{i} y_{i}} .
$$

The Leontief inverse is

$$
\Psi=(I-\Omega)^{-1} .
$$

Intuitively, the $i j$ th element $\Psi_{i j}$ of the Leontief inverse is a measure of $i$ 's total reliance on $j$ as a supplier. It captures both the direct and indirect ways through which $i$ uses $j$ in its production. ${ }^{30}$

Definition 4. The input-output covariance operator is

$$
\operatorname{Cov}_{\Omega^{(k)}}\left(\Psi_{(i)}, \Psi_{(j)}\right)=\sum_{l=1}^{N+F} \Omega_{k l} \Psi_{l i} \Psi_{l j}-\left(\sum_{l=1}^{N+F} \Omega_{k l} \Psi_{l i}\right)\left(\sum_{l=1}^{N+F} \Omega_{k l} \Psi_{l j}\right) .
$$

\footnotetext{
${ }^{30}$ See, for example, Baqaee (2015) for a detailed description of $\Omega$ and $\Psi$.
} 
It is the covariance between the $i$ th and $j$ th column of the Leontief inverse using the $k$ th row of the input-output matrix as the distribution. The input-output covariance operator plays a crucial role in our results.

We consider arbitrary CES network structures (in standard-form), starting with a single factor and then generalizing to multiple factors. As previously mentioned, a one-factor model is equivalent to a model where primary factors are equivalent and can be fully reallocated. To model limited factor reallocation or decreasing-returns, we need to have multiple factors.

\subsection{One Factor}

Proposition 7 (Second-Order Network Centrality). Consider a nested-CES model in standard form with a single factor. The second-order macroeconomic impact of microeconomic shocks is given by

$$
\frac{\mathrm{d}^{2} \log Y}{\mathrm{~d} \log A_{j} \mathrm{~d} \log A_{i}}=\frac{\mathrm{d} \lambda_{i}}{\mathrm{~d} \log A_{j}}=\sum_{k=0}^{N}\left(\theta_{k}-1\right) \lambda_{k} \operatorname{Cov}_{\Omega^{(k)}}\left(\Psi_{(i)}, \Psi_{(j)}\right),
$$

and in particular

$$
\frac{\mathrm{d}^{2} \log Y}{\mathrm{~d} \log A_{i}^{2}}=\frac{\mathrm{d} \lambda_{i}}{\mathrm{~d} \log A_{i}}=\sum_{k=0}^{N}\left(\theta_{k}-1\right) \lambda_{k} \operatorname{Var}_{\Omega^{(k)}}\left(\Psi_{(i)}\right)
$$

Equations (6) and (7) have a simple intuition. Let us focus first on equation (6). The change in the sales share of $i$, in response to a shock to $j$, depends on how the relative demand expenditure for $i$ changes. Changes in the demand expenditure for $i$ arise from the substitution by the different nodes $k$ and captured by the different terms in the sum on the right-hand side. ${ }^{31}$

Consider for example the effect of a negative productivity shock $\mathrm{d} \log A_{j}<0$ to $j$. The change in the vector of prices of the different producers is proportional to the vector of direct and indirect exposures to the shock, which is simply the $j$ th column $\Psi_{(j)}$ of the Leontief inverse. Now consider a given producer $k$. If $\theta_{k}<1$, producer $k$ increases its expenditure share on inputs whose price increases more, i.e. inputs that are more exposed to the shock to $j$, as measured by $\Psi_{(j)}$. This increases the relative demand expenditure for $i$ if those inputs are also relatively more exposed to $i$, as measured by the $i$ th column of the Leontief inverse $\Psi_{(i)}$. The overall effect is stronger, the higher is the covariance $\operatorname{Cov}_{\Omega^{(k)}}\left(\Psi_{(i)}, \Psi_{(j)}\right)$, the larger is

\footnotetext{
${ }^{31}$ In ongoing work (Baqaee and Farhi, 2019), we show that there is a connection between these formulas and the gains-from-trade formulas in Arkolakis et al. (2012). See Appendix F for more details.
} 
the size of producer $k$ as measured by $\lambda_{k}$, and the further away from one is the elasticity of substitution $\theta_{k}$ as measured by $\theta_{k}-1$.

Equation (7) is a particular case of equation (6) and so the intuition is identical. The change in the sales share of $i$ depends on substitution by all producers $k$. The extent to which substitution by producer $k$ matters depends on how unequally $k$ is exposed to $i$ through its different inputs, on how large $k$ is, and on far away from one is the elasticity of substitution in production of $k$. If $k$ is small, or is exposed in the same way to $i$ through all of its inputs, then the extent to which it can substitute amongst its inputs is irrelevant. If the elasticity of substitution of $k$ is equal to one, then the direct and indirect relative demand expenditure for $i$ arising from $k$ does not change in response to shocks. Equation (7) can be seen as a centrality measure which combines structural microeconomic elasticities of substitution and features of the network. ${ }^{32}$

The Cobb-Douglas specification is the knife-edge special case where all the second-order terms are equal to zero and where the first-order approximation is globally accurate. This occurs because Domar weights, and more generally, the whole input-output matrix, are constant and can be taken to be exogenous. Away from the Cobb-Douglas case, sales shares and the input-output matrix respond endogenously to shocks, and this is precisely what gives rise to the nonlinearities which are captured by the second-order approximation.

\section{GE Elasticities of Substitution}

Proposition 7 can also be used to compute the GE elasticities of substitution, using equations (6) and (7) to substitute the corresponding derivatives in the following equations:

$$
1-\frac{1}{\rho_{j i}}=\frac{\mathrm{d} \log \lambda_{i}}{\mathrm{~d} \log A_{i}}-\frac{\mathrm{d} \log \lambda_{j}}{\mathrm{~d} \log A_{i}}
$$

and

$$
\frac{\mathrm{d} \log \xi}{\mathrm{d} \log A_{i}}=\frac{1}{\xi} \sum_{j} \lambda_{j} \frac{\mathrm{d} \log \lambda_{j}}{\mathrm{~d} \log A_{i}} .
$$

\section{A Network-Irrelevance Result}

To build more intuition, we provide a benchmark irrelevance result where the deviation from Hulten's approximation does not depend on the network structure. ${ }^{33}$ The key assumptions required for obtaining this irrelevance result are: (1) productivity shocks are

\footnotetext{
${ }^{32}$ Equation (7) is also related to the concentration centrality defined by Acemoglu et al. (2016), but generalizes their result by allowing for heterogeneity in the interaction functions, non-symmetric network structures, and micro-founds its use for production networks.

${ }^{33}$ Footnote 13 of Baqaee (2018) also discusses this network irrelevance result.
} 
factor-augmenting; (2) the structural microeconomic elasticities of substitution are all the same.

Once the economy is written in standard form, the shocks are factor-augmenting if they only hit producers $i$ which have the primary factor as their only input, i.e. if $\Omega_{i j}=0$ for every $j=0, \ldots, N$. In other words, the productivity shocks $A_{i}$ hitting producers $i$ which do not have the primary factor as their only input are kept at their steady-state values of $A_{i}=1$.

Corollary 1 (Network Irrelevance). Consider a nested-CES model in standard form with a single factor, uniform elasticities of substitution $\theta_{j}=\theta$ for every $j$, and with factor-augmenting shocks. Aggregate output is given by the closed-form expression

$$
\frac{Y}{\bar{Y}}=\left(\sum_{i=0}^{N} \bar{\lambda}_{i} A_{i}^{\theta-1}\right)^{\frac{1}{\theta-1}}
$$

where $\bar{\lambda}_{i}$ is the steady-state Domar weight of $i$. The second-order macroeconomic impact of factoraugmenting microeconomic shocks is given by

$$
\frac{\mathrm{d}^{2} \log Y}{\mathrm{~d} \log A_{j} \mathrm{~d} \log A_{i}}=\frac{\mathrm{d} \lambda_{i}}{\mathrm{~d} \log A_{j}}=(\theta-1) \lambda_{i}\left(\mathbf{1}(i=j)-\lambda_{j}\right)
$$

and in particular

$$
\frac{\mathrm{d}^{2} \log Y}{\mathrm{~d} \log A_{i}^{2}}=\frac{\mathrm{d} \lambda_{i}}{\mathrm{~d} \log A_{i}}=\sum_{j=0}^{N}\left(\theta_{j}-1\right) \lambda_{j} \operatorname{Var}_{\Omega^{(j)}}\left(\Psi_{(i)}\right)=(\theta-1) \lambda_{i}\left(1-\lambda_{i}\right) .
$$

In words, if we consider factor-augmenting shocks, and if all microeconomic elasticities of substitution are the same, then the network structure remains irrelevant, even though there are deviations from Hulten's approximation. In this special case, the Domar weights and the structural microeconomic elasticities of substitution are sufficient statistics for the second-order effects.In fact, the result is true not only locally, but also globally.

Essentially, factor-augmenting shocks shut down variations in $\xi$, which is constant and equal to one, and uniform structural microeconomic elasticities of substitution shut down variations in $\rho_{j i}$ which are uniform and constant

$$
\rho_{j i}=\frac{1}{2-\theta} .
$$

Deviating from either condition breaks the irrelevance. 


\section{Energy Example - One Factor}

A simple example, motivated by a universal intermediate input like energy, helps explain some of the intuition of Proposition 7 and Corollary 1. Consider the example economy depicted in Figure 3. Energy is produced linearly from labor:

$$
\frac{e}{\bar{e}}=A_{e} \frac{l_{e}}{\bar{l}_{e}} .
$$

Downstream producers produce using energy and labor with elasticity of substitution $\theta_{1}$ :

$$
\frac{y_{i}}{\bar{y}_{i}}=\left(\left(1-\omega_{i e}\right) \frac{l_{l_{1}}^{\frac{\theta_{1}-1}{\theta_{1}}}}{\bar{l}_{i}}+\omega_{i e}\left(\frac{e_{i}}{\bar{e}_{i}}\right)^{\frac{\theta_{1}-1}{\theta_{1}}}\right)^{\frac{\theta_{1}}{\theta_{1}-1}} .
$$

They sell directly to the household who values goods with an elasticity of substitution $\theta_{0}$ :

$$
\frac{Y}{\bar{Y}}=\left(\sum_{i=1}^{N} \omega_{0 i}\left(\frac{c_{i}}{\bar{c}_{i}}\right)^{\frac{\theta_{0}-1}{\theta_{0}}}\right)^{\frac{\theta_{0}}{\theta_{0}-1}}
$$

where $\sum_{i=1}^{N} \omega_{0 i}=1$.

The market-clearing conditions are

$$
y_{i}=c_{i}, \quad e=\sum_{i=1}^{N} e_{i} \quad \text { and } \quad \bar{l}=l_{e}+\sum_{i=1}^{N} l_{i},
$$

where $\bar{l}=\bar{l}_{e}+\sum_{i=1}^{N} \bar{l}_{i}$.

Producer $i^{\prime}$ 's steady-state sales share is $\lambda_{i}=\omega_{0 i}$, the intermediate input share of industry $i$ is $\omega_{i e}$, and the sales share of energy is $\lambda_{e}=\sum_{i} \lambda_{i} \omega_{i e}$.

We simplify the example further by supposing that all final sectors are equally sized with $\lambda_{i}=1 / N$, and that $M \leq N$ producers use energy with the same steady-state intermediate input share $\omega_{i e}=\omega_{e}$, while the other $N-M$ producers use no energy at all so that $\omega_{i e}=0$. We set $\omega_{e}$ to ensure that $\lambda_{e}$ stays constant. We take $\theta_{1}<\theta_{0}$ and $\theta_{1}<1$.

Proposition 7 implies that

$$
\begin{aligned}
\frac{\mathrm{d}^{2} \log Y}{\mathrm{~d} \log A_{e}^{2}} & =\lambda_{e}^{2} \frac{N-M}{N}\left(\theta_{0}-1\right)+\lambda_{e}\left(1-\frac{N}{M} \lambda_{e}\right)\left(\theta_{1}-1\right) \\
& =\lambda_{e}\left(1-\lambda_{e}\right)\left(\theta_{0}-1\right)+\lambda_{e}\left(1-\frac{N}{M} \lambda_{e}\right)\left(\theta_{1}-\theta_{0}\right) .
\end{aligned}
$$




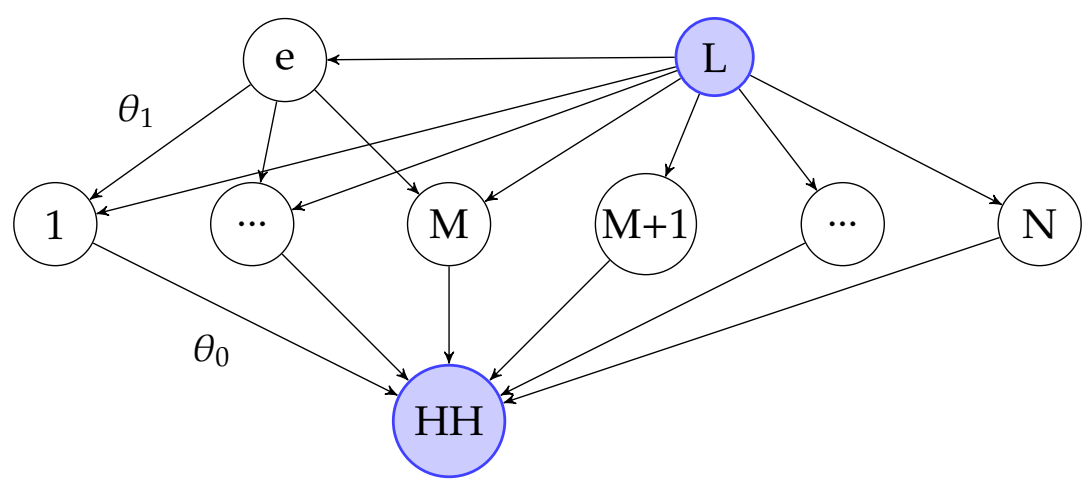

Figure 3: An illustration of the economy with a near-universal intermediate input which we call energy. Each downstream producer substitutes across labor and energy with elasticity $\theta_{1}<1$. The household can substitute across final goods with elasticity of substitution $\theta_{0}>\theta_{1}$. Energy is produced from labor with constant-returns.

The first equation directly expresses the second-order term as a weighted sum of the microeconomic elasticities of substitution as in Proposition 7. The first term on the right-hand side of the second equation is the network-independent second-order term when all the microeconomic elasticities of substitution are identical so that $\theta_{0}=\theta_{1}$ as in Corollary 1 . The second term is a network-dependent correction that takes into account the fact that $\theta_{0} \neq \theta_{1}$.

When every sector uses energy $M=N$, these equations become

$$
\frac{\mathrm{d}^{2} \log Y}{\mathrm{~d} \log A_{e}^{2}}=\lambda_{e}\left(1-\lambda_{e}\right)\left(\theta_{1}-1\right),
$$

and the elasticity of substitution in consumption $\theta_{0}$ drops out completely. The fact that $\theta_{0}$ is irrelevant when $M=N$ is a manifestation of the general principle stated in Proposition 7. When $M=N$, energy is a universal input and hence $\operatorname{Var}_{\Omega^{(0)}}\left(\Psi_{(e)}\right)=0$. In this case, the household is symmetrically exposed to shocks to energy via the different downstream producers, and so the elasticity of substitution in consumption $\theta_{0}$ is irrelevant.

When $M \neq N$ instead $\theta_{0}$ matters, with a weight that decreases with $N$. Through the lens of Proposition 7, $\operatorname{Var}_{\Omega^{(0)}}\left(\Psi_{(e)}\right)=\lambda_{e}^{2}(N-M) / N>0$ is decreasing in $M$ : as heterogeneity in energy-intensity across downstream producers increases, the ability of the household to substitute across these producers matters more and more.

Because $\theta_{1}<1$, when $M=N$, the second-order approximation magnifies negative the macroeconomic impact of negative shocks to energy compared to the first-order approximation. As $M$ decreases, this effect becomes weaker, since a lower $M$ means that energy is less of a universal input, and so it becomes easier to substitute away from it further downstream across producers with different energy intensities. The sign of the effect can even flip if $M$ is low enough and if $\theta_{0}$ is high enough above one. 


\section{Macro-Influence - One Factor}

A final implication of Proposition 7 is that it is only the producer's role as a supplier that matters, not its role as a consumer. ${ }^{34}$

Proposition 8 (Macro-Influence). Consider a nested-CES model in standard form with a single factor. Suppose that all producers $k$ have the same expenditures on producers $i$ and $j$ so that $\Omega_{k i}=\Omega_{k j}$ for all $k$. Then

$$
\begin{aligned}
& \frac{\mathrm{d} \log Y}{\mathrm{~d} \log A_{i}}=\frac{\mathrm{d} \log Y}{\mathrm{~d} \log A_{j}}, \\
& \frac{\mathrm{d}^{2} \log Y}{\mathrm{~d} \log A_{i}^{2}}=\frac{\mathrm{d}^{2} \log Y}{\mathrm{~d} \log A_{j}^{2}},
\end{aligned}
$$

and for all $l$,

$$
\frac{\mathrm{d}^{2} \log Y}{\mathrm{~d} \log A_{l} \mathrm{~d} \log A_{i}}=\frac{\mathrm{d}^{2} \log Y}{\mathrm{~d} \log A_{l} \mathrm{~d} \log A_{j}} .
$$

The intuition is that, in a one factor model, we can normalize the wage to one, and then aggregate output, which is equal to real factor income, depends only on the prices of final goods. A change in the size of the ith industry does not affect its price. Hence, a productivity shock travels downstream from suppliers to their consumers by lowering their marginal costs and hence their prices, but it does not travel upstream from consumers to their suppliers. This result fails whenever there are multiple factors, and by implication when the model does not feature constant returns to scale. ${ }^{35}$

\subsection{Multiple Factors}

We now generalize the results of the previous section to allow for multiple factors of production. This in turn opens the door to modelling limited-reallocation and decreasing-returnsto-scale via producer and industry-specific fixed factors.

We sometime use separate uppercase indices to denote the producers that correspond to factors, and lowercase indices to denote all other producers. For example, we sometime use $\Lambda_{f}$ to denote the Domar weight, or income share, of factor $f$, and $\Lambda$ to denote the $F \times 1$ vector of factor shares.

\footnotetext{
${ }^{34}$ This generalizes a result in Baqaee (2018).

${ }^{35}$ Acemoglu et al. (2015) show that outside of the Cobb-Douglas special case, shocks can propagate both upstream and downstream. There is no contradiction between their result and Proposition 8 . Their results and ours simply characterize different forms of propagation: they focus on the propagation of shocks to producers on the quantities and sales of other producers, whereas we focus on the impact of shocks to producers on the prices of other producers and on aggregate output.
} 
Proposition 9 (Second-Order Network Centrality with Multiple Factors). Consider a nestedCES model in standard form. Then

$$
\begin{aligned}
\frac{\mathrm{d}^{2} \log Y}{\mathrm{~d} \log A_{j} \mathrm{~d} \log A_{i}} & =\frac{\mathrm{d} \lambda_{i}}{\mathrm{~d} \log A_{j}}=\sum_{k=0}^{N}\left(\theta_{k}-1\right) \lambda_{k} \operatorname{Cov}_{\Omega^{(k)}}\left(\Psi_{(j)}, \Psi_{(i)}\right) \\
& -\sum_{f=N+1}^{N+F} \frac{\mathrm{d} \log \Lambda_{f}}{\mathrm{~d} \log A_{j}} \sum_{k=0}^{N}\left(\theta_{k}-1\right) \lambda_{k} \operatorname{Cov}_{\Omega^{(k)}}\left(\Psi_{(f)}, \Psi_{(i)}\right),
\end{aligned}
$$

where the vector of elasticities of the factor income shares to the shocks solves the linear system

$$
\frac{\mathrm{d} \log \Lambda}{\mathrm{d} \log A_{j}}=\Gamma \frac{\mathrm{d} \log \Lambda}{\mathrm{d} \log A_{j}}+\delta^{(j)},
$$

with

$$
\Gamma_{f, g}=-\frac{1}{\Lambda_{f}}\left(\sum_{k=0}^{N}\left(\theta_{k}-1\right) \lambda_{k} \operatorname{Cov}_{\Omega^{(k)}}\left(\Psi_{(f)}, \Psi_{(g)}\right)\right),
$$

and

$$
\delta_{f}^{(j)}=\frac{1}{\Lambda_{f}}\left(\sum_{k=0}^{N}\left(\theta_{k}-1\right) \lambda_{k} \operatorname{Cov}_{\Omega^{(k)}}\left(\Psi_{(f)}, \Psi_{(j)}\right)\right) .
$$

Note that we can rewrite equation (8) as a function of $\mathrm{d} \log \lambda_{j} / \mathrm{d} \log A_{j}$ using the identity $\mathrm{d} \log \lambda_{j} / \mathrm{d} \log A_{j}=\left(1 / \lambda_{j}\right)\left(\mathrm{d} \lambda_{j} / \mathrm{d} \log A_{j}\right)$. Proposition 9 can then be seen as a full characterization of the elasticities of the Domar weights of the different producers to the different shocks.

The intuition is the following. The first set of summands on the right-hand side of equation (8) are exactly those in equation (6) in Proposition 7: these terms capture how substitution by downstream producers $k$ in response to a shock to $j$ changes the sales share of $i$. The second set of summands in equation (8) take into account the fact that, when there are multiple factors, the shock also changes relative factor prices, and substitution in response to changes in factor prices in turn affects the sales share of $i$.

Consider for example a negative shock $\mathrm{d} \log A_{j}<0$ to producer $j$. Imagine that this shock increases the price of factor $f$ relative to the prices of other factors, so that $\mathrm{d} \log \Lambda_{f}>0$. Now consider the response of a producer $k$ to this change. If $\theta_{k}<1$, producer $k$ increases its expenditure share on producers that are more exposed to factor $f$ as measured by $\Psi_{(f)}$. If these producers are also more exposed to $i$, as measured by $\Psi_{(i)}$, then the substitution increases the sales share of $i$. These changes must be cumulated across producers $k$ and factors $f$. The total effect on the relative demand expenditure for producer $i$, and hence on its sales share, is the sum of the effect of substitutions in response to the initial impulse 
$\mathrm{d} \log A_{j}$, as well as the substitutions in responses to changes in relative factor prices captured by $\mathrm{d} \log \Lambda_{f}$.

Equation (9) in turn determines how factor shares $\mathrm{d} \log \Lambda_{f} / \mathrm{d} \log A_{j}$ respond to different shocks. For a given set of factor prices, a shock to $j$ affects the relative demand expenditure for each factor, and hence the factor income shares, as measured by the $F \times 1$ vector $\delta^{(j)}$. This change in the factor income shares then causes further substitution through the network, leading to additional changes in relative factor shares and prices. The impact of the change in the relative share or price of factor $g$ on the relative demand expenditure for factor $f$ is measured by the $f g$ th element of the $F \times F$ matrix $\Gamma$. Crucially, the matrix $\Gamma$ does not depend on which producer $j$ is being shocked.

We can verify that we get back Proposition 7 when there is only a single factor, since in that case the exposure vector $\Psi_{(f)}$, corresponding to the unique factor, is equal to a vector of all ones, and so the second set of summands in equation (8) is identically zero.

Just like in the case of a single factor and for the same reasons, the Cobb-Douglas specification is the knife-edge special case where all the second-order terms are equal to zero and where the first-order approximation is globally accurate because sales shares, and more generally the whole input-output matrix, are constant and can be taken to be exogenous.

\section{GE Elasticities of Substitution}

Proposition 9 can also be used to compute the GE elasticities of substitution, using equations (6) and (7) to substitute the corresponding derivatives in the following equations: ${ }^{36}$

$$
1-\frac{1}{\rho_{j i}}=\frac{\mathrm{d} \log \lambda_{i}}{\mathrm{~d} \log A_{i}}-\frac{\mathrm{d} \log \lambda_{j}}{\mathrm{~d} \log A_{i}}
$$

and

$$
\frac{\mathrm{d} \log \xi}{\mathrm{d} \log A_{i}}=\frac{1}{\xi} \sum_{j} \lambda_{j} \frac{\mathrm{d} \log \lambda_{j}}{\mathrm{~d} \log A_{i}}
$$

\footnotetext{
${ }^{36}$ Although Proposition 9 is stated in terms of productivity shocks to non-factor producers $j$, the same formulas hold for a productivity shock $\mathrm{d} \log A_{g}$ to a factor industry $g$. The productivity shock is then just a shock to the endowment of the factor. ${ }^{37}$ We can also compute the GE elasticities of substitution between two factors $g$ and $f$ using

$$
1-\frac{1}{\rho_{g f}}=\frac{\mathrm{d} \log \Lambda_{f}}{\mathrm{~d} \log A_{f}}-\frac{\mathrm{d} \log \Lambda_{g}}{\mathrm{~d} \log A_{f}} .
$$

We refer the reader to Baqaee and Farhi (2018a) for an extensive discussion of macroeconomic elasticities of substitution between factors. This paper also characterizes the macroeconomic bias of technical change as a function of microeconomic primitives.
} 


\section{A Network-Irrelevance Result}

In the special case where all microeconomic elasticities of substitution are the same, we once again obtain network-irrelevance result. However, because there are multiple factors, it is not enough to consider factor-augmenting shocks as we did in the case of a single factor, and we must instead focus on shocks that increase the overall quantities of the different factors.

Corollary 2 (Network Irrelevance). Consider a nested-CES model in standard form with uniform elasticities of substitution $\theta_{j}=\theta$ for every $j$, and shocks $A_{f}$ to the supplies of the different factors $f$. Aggregate output is given by the following closed-form expression

$$
\frac{Y}{\bar{Y}}=\left(\sum_{f=N+1}^{N+F} \bar{\Lambda}_{f} A_{f}^{\frac{\theta-1}{\theta}}\right)^{\frac{\theta}{\theta-1}}
$$

where $\bar{\Lambda}_{f}$ is the Domar weight of $f$ at steady-state. The second-order macroeconomic impact of microeconomic shocks to the supplies of factors is given by

$$
\frac{\mathrm{d}^{2} \log Y}{\mathrm{~d} \log A_{g} \mathrm{~d} \log A_{f}}=\frac{\mathrm{d} \Lambda_{f}}{\mathrm{~d} \log A_{g}}=\left(1-\frac{1}{\theta}\right) \Lambda_{g}\left(\mathbf{1}(g=f)-\Lambda_{g}\right)
$$

and in particular,

$$
\frac{\mathrm{d}^{2} \log Y}{\mathrm{~d} \log A_{f}^{2}}=\frac{\mathrm{d} \Lambda_{f}}{\mathrm{~d} \log A_{f}}=\left(1-\frac{1}{\theta}\right) \Lambda_{f}\left(1-\Lambda_{f}\right) .
$$

In this special case, the Domar weight and the structural microeconomic elasticities of substitution are sufficient statistics for the second-order effects. In fact, the result is true not only locally, but also globally.

A consequence of this corollary is that whenever all the micro-elasticities of substitution are the same and equal to $\theta$, the GE elasticity of substitution $\rho_{g f}$ between any two factors $f$ and $g$ is also equal to $\theta$.

\section{Energy Example - Multiple Factors}

We revisit the energy example introduced in Section 4.1, but we now model energy as an endowment rather than as a produced good. The economy is represented in Figure 4. For this example, the effect of a shock to energy is now a nonlinear function of the underlying microeconomic elasticities of substitution

$$
\frac{\mathrm{d}^{2} \log Y}{\mathrm{~d} \log A_{e}^{2}}=\frac{\mathrm{d} \Lambda_{e}}{\mathrm{~d} \log A_{e}^{2}}=\frac{\left(\theta_{0}-1\right) \Lambda_{e}\left(1-\Lambda_{e}\right)+\left(\theta_{1}-\theta_{0}\right) \Lambda_{e}\left(1-\frac{N}{M} \Lambda_{e}\right)}{\theta_{0}+\left(\theta_{1}-\theta_{0}\right) \frac{1-\frac{N}{M} \Lambda_{e}}{1-\Lambda_{e}}} .
$$




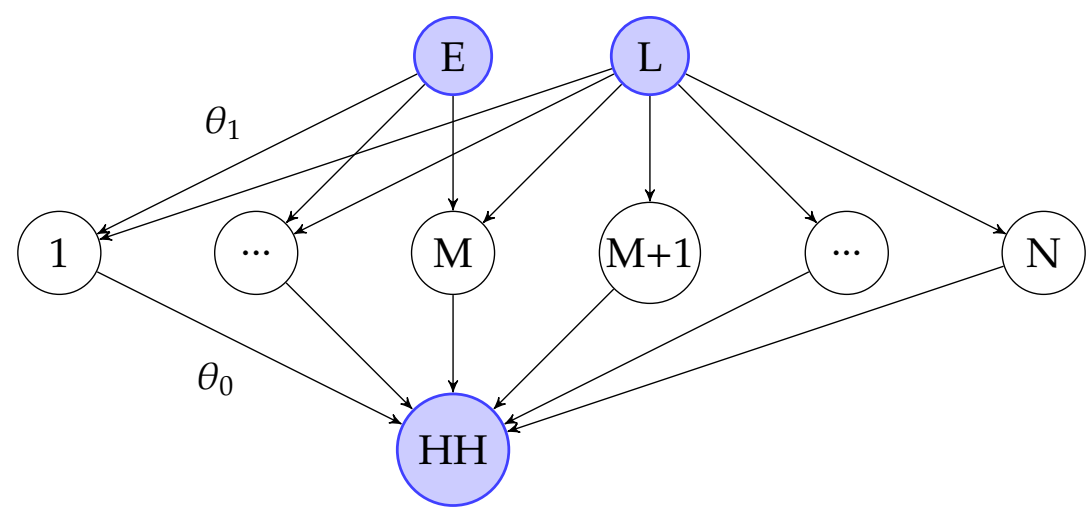

Figure 4: An illustration of the economy with a near-universal input which we treat as energy. Each industry has different shares of labor and energy and substitutes across labor and energy with elasticity $\theta_{1}<1$. The household can substitute across goods with elasticity of substitution $\theta_{0}>\theta_{1}$. Labor and energy are in fixed supply.

The difference with the case of a single factor is that following a negative productivity shock to energy, labor cannot be reallocated to the production of energy in order to reinforce that weak link. This effect, encapsulated in the denominator, further amplifies the effect of the shock.

Once again, and in accordance with Corollary 2, whenever the micro-elasticities of substitution are the same $\theta_{0}=\theta_{1}$, the shape of the network becomes irrelevant. Additionally, in the extreme case where energy becomes a universal input $M=N$, the elasticity $\theta_{0}$ drops out of the formula because producers are uniformly exposed to energy:

$$
\frac{\mathrm{d}^{2} \log Y}{\mathrm{~d} \log A_{e}^{2}}=\left(1-\frac{1}{\theta_{1}}\right) \Lambda_{e}\left(1-\Lambda_{e}\right)
$$

Note that even in this case, the formula is different from that of the case of a single factor where the first term on the right-hand side is $\theta_{1}-1$ instead of $1-1 / \theta_{1}$. This reflects the aforementioned fact that in contrast to the case of a single factor, labor cannot be reallocated to the production of energy following a negative shock to energy, which further amplifies the negative impact of the energy shock.

\section{Macro-Influence - Multiple Factors}

In contrast to the case of a single factor, shocks to prices now propagate downstream and upstream. The result derived in Proposition 8 when there is only factor breaks down when there are multiple factors: two producers with identical demand chains do not necessarily have the same importance. This is because they might have different direct and indirect exposures to the different factors. As a result, the role of a producer as a consumer matters 
in addition to its role as a supplier.

\section{Beyond CES}

The input-output covariance operator defined in equation (5) is a key concept capturing the substitution patterns in economies where all production and utility functions are nestedCES functions. In this section, we generalize this input-output covariance operator in such a way that allows us to work with arbitrary production functions.

For a producer $k$ with cost function $\mathbf{C}_{k}$, we define the Allen-Uzawa elasticity of substitution between inputs $x$ and $y$ as

$$
\theta_{k}(x, y)=\frac{\mathbf{C}_{k} d^{2} \mathbf{C}_{k} /\left(d p_{x} d p_{y}\right)}{\left(d \mathbf{C}_{k} / d p_{x}\right)\left(d \mathbf{C}_{k} / d p_{y}\right)}=\frac{\epsilon_{k}(x, y)}{\Omega_{k y}}
$$

where $\epsilon_{k}(x, y)$ is the elasticity of the demand by producer $k$ for input $x$ with respect to the price $p_{y}$ of input $y$, and $\Omega_{k y}$ is the expenditure share in cost of input $y$.

Note the following properties. Because of the symmetry of partial derivatives, we have $\theta_{k}(x, y)=\theta_{k}(y, x)$. Because of the homogeneity of degree one of the cost function in the prices of inputs, we have the homogeneity identity $\sum_{1 \leq y \leq N+1+F} \Omega_{k y} \theta_{k}(x, y)=0$.

We define the input-output substitution operator for producer $k$ as

$$
\begin{aligned}
\Phi_{k}\left(\Psi_{(i)}, \Psi_{(j)}\right) & =-\sum_{1 \leq x, y \leq N+1+F} \Omega_{k x}\left[\delta_{x y}+\Omega_{k y}\left(\theta_{k}(x, y)-1\right)\right] \Psi_{x i} \Psi_{y j}, \\
& =\frac{1}{2} E_{\Omega^{(k)}}\left(\left(\theta_{k}(x, y)-1\right)\left(\Psi_{i}(x)-\Psi_{i}(y)\right)\left(\Psi_{j}(x)-\Psi_{j}(y)\right)\right),
\end{aligned}
$$

where $\delta_{x y}$ is the Kronecker delta, $\Psi_{i}(x)=\Psi_{x i}$ and $\Psi_{j}(x)=\Psi_{x j}$, and the expectation on the second line is over $x$ and $y$. The second line can be obtained from the first using the symmetry of Allen-Uzawa elasticities of substitution and the homogeneity identity.

In the CES case with elasticity $\theta_{k}$, all the cross Allen-Uzawa elasticities are identical with $\theta_{k}(x, y)=\theta_{k}$ if $x \neq y$, and the own Allen-Uzawa elasticities are given by $\theta_{k}(x, x)=$ $-\theta_{k}\left(1-\Omega_{k x}\right) / \Omega_{k x}$. It is easy to verify that we then recover the input-output covariance operator:

$$
\Phi_{k}\left(\Psi_{(i)}, \Psi_{(j)}\right)=\left(\theta_{k}-1\right) \operatorname{Cov}_{\Omega^{(k)}}\left(\Psi_{(i)}, \Psi_{(j)}\right) .
$$

Even outside the CES case, the input-output substitution operator shares many properties with the input-output covariance operator. For example, it is immediate to verify, that: $\Phi_{k}\left(\Psi_{(i)}, \Psi_{(j)}\right)$ is bilinear in $\Psi_{(i)}$ and $\Psi_{(j)} ; \Phi_{k}\left(\Psi_{(i)}, \Psi_{(j)}\right)$ is symmetric in $\Psi_{(i)}$ and $\Psi_{(j)}$; and $\Phi_{k}\left(\Psi_{(i)}, \Psi_{(j)}\right)=0$ whenever $\Psi_{(i)}$ or $\Psi_{(j)}$ is a constant. 
Luckily, it turns out that all of the results stated so far can be generalized to non-CES economies simply by replacing terms of the form $\left(\theta_{k}-1\right) \operatorname{Cov}_{\Omega^{(k)}}\left(\Psi_{(i)}, \Psi_{(j)}\right)$ by $\Phi_{k}\left(\Psi_{(i)}, \Psi_{(j)}\right)$. For example, equation (8) in Proposition 9 becomes

$$
\frac{\mathrm{d}^{2} \log Y}{\mathrm{~d} \log A_{j} \mathrm{~d} \log A_{i}}=\sum_{k=0}^{N} \Phi_{k}\left(\Psi_{(i)}, \Psi_{(j)}\right)-\sum_{f=N+1}^{N+F} \frac{\mathrm{d} \log \Lambda_{f}}{\mathrm{~d} \log A_{j}} \sum_{k=0}^{N} \Phi_{k}\left(\Psi_{(i)}, \Psi_{(f)}\right) .
$$

By replacing the input-output covariance operator with the input-output substitution operator, we fully characterize the Hessian of the output function of the general economy described in Section 2, with arbitrary, and potentially, non-homothetic production functions, an arbitrary number of factors, and arbitrary patterns of input-output linkages.

Intuitively, $\Phi_{k}\left(\Psi_{(i)}, \Psi_{(j)}\right)$ captures the way in which $k$ redirects demand expenditure towards $i$ in response to proportional unit decline in the price of $j$. To see this, we make use of the following observation: the elasticity of the expenditure share of producer $k$ on input $x$ with respect to the price of input $y$ is given by $\delta_{x y}+\Omega_{k y}\left(\theta_{k}(x, y)-1\right)$. Equation (11) requires considering, for each pair of inputs $x$ and $y$, how much the proportional reduction $\Psi_{y j}$ in the price of $y$ induced by a unit proportional reduction in the price of $j$ causes producer $k$ to increase its expenditure share on $x$ (as measured by $-\Omega_{k x}\left[\delta_{x y}+\Omega_{k y}\left(\theta_{k}(x, y)-1\right)\right] \Psi_{y j}$ ) and how much $x$ is exposed to $i$ (as measured by $\Psi_{x i}$ ).

Equation (12) says that this amounts to considering, for each pair of inputs $x$ and $y$, whether or not increased exposure to $j$ as measured by $\Psi_{j}(x)-\Psi_{j}(y)$, corresponds to increased exposure to $i$ as measured by $\Psi_{i}(x)-\Psi_{i}(y)$, and whether $x$ and $y$ are complements or substitutes as measured by $\left(\theta_{k}(x, y)-1\right)$. If $x$ and $y$ are substitutes, and $\Psi_{j}(x)-\Psi_{j}(y)$ and $\Psi_{i}(x)-\Psi_{i}(y)$ are both positive, then substitution across $x$ and $y$ by $k$, in response to a shock to a decrease in the price of $j$, increases demand for $i$.

\section{Quantitative Illustration}

In this section, we develop some illustrative quantitative applications of our results to gauge the practical importance of the nonlinearities that we have identified. We perform three exercises focusing on macroeconomic phenomena at different frequencies. First, we calibrate a multi-sector business-cycle model with sectoral productivity shocks. We match the observed input-output data, and use the best available information to choose the structural (micro) elasticities of substitution, and we match the volatility of sectoral shocks at business-cycle frequencies. We compare the outcome of the nonlinear model to its first-order approximation. In the second exercise, we study the macroeconomic impact of the energy crisis of the 1970s using a non-parametric generalization of Hulten (1978) that takes second-order terms 
into account. In the third and final exercise, we investigate the importance of nonlinearities which underpin Baumol's cost disease for long-run aggregate TFP growth. All our exercises suggest that production is highly nonlinear.

\subsection{A Quantitative Multi-Sector Business-Cycle Model with Sectoral Pro- ductivity Shocks}

In this section, we use a simple version of the structural model defined in Section 2, calibrate it with sectoral shocks at business-cycle frequencies, and solve for the equilibrium using global solution methods. ${ }^{38}$ The final demand function is

$$
\frac{Y}{\bar{Y}}=\left(\sum_{i=1}^{N} \omega_{0 i}\left(\frac{c_{i}}{\bar{c}_{i}}\right)^{\frac{\sigma-1}{\sigma}}\right)^{\frac{\sigma}{\sigma-1}}
$$

The production function of industry $i$ is

$$
\frac{y_{i}}{\bar{y}_{i}}=A_{i}\left(\omega_{i l}\left(\frac{l_{i}}{\bar{l}_{i}}\right)^{\frac{\theta-1}{\theta}}+\left(1-\omega_{i l}\right)\left(\frac{\hat{X}_{i}}{\bar{X}_{i}}\right)^{\frac{\theta-1}{\theta}}\right)^{\frac{\theta}{\theta-1}}
$$

consisting of labor inputs $l_{i}$ and intermediate inputs $\hat{X}_{i}$.

We consider two polar opposite possibilities for the labor market: the case where each labor type is specific to each industry, and cannot be reallocated, and the case where there is a common factor which can be reallocated across all industries. In light of increasing evidence (see for example Acemoglu et al., 2016; Autor et al., 2016; Notowidigdo, 2011) that labor is not easily reallocated across industries or regions after shocks in the short run, we view the no-reallocation case as more realistic for modeling the short-run impact of shocks, and the full-reallocation case as better suited to study the medium to long-run impact shocks. ${ }^{39}$

\footnotetext{
${ }^{38}$ For comparison, Table 3 in Appendix D shows the macro-moments for the model using the second-order approximation. The second-order approximation does a very good job at capturing the mean and standard deviation of aggregate output at both annual and quadrennial frequencies. It also performs well for skewness and kurtosis at an annual frequency, but less so at a quadrennial frequency. Basically, the quality of the approximation is worse for larger shocks and higher moments of output. Of course, whenever it is feasible to solve the model non-linearly, the fully nonlinear solution is preferable. However, even in these cases, our analytical pen-and-paper approach also sheds light on the mechanisms driving nonlinearities that would be lost were we to simply solve a series of nonlinear equations on a computer. Furthermore, our sufficient statistics approach allows us to construct the second-order approximation without needing to fully specify the nonlinear model.

${ }^{39}$ In our baseline calibrations, we assume that intermediate inputs can be freely reallocated across producers even in the short run. This is sensible since intermediate goods are probably easier to reallocate than labor. We refer the reader to Appendix $C$ for a version of these calibrations in the presence of costs of adjusting intermediate inputs. These adjustment costs hamper the reallocation of intermediate inputs across producers
} 
The composite intermediate input $X_{i}$ is given by

$$
\frac{X_{i}}{\bar{X}_{i}}=\left(\sum_{j=1}^{N} \omega_{i j}\left(\frac{x_{i j}}{\overline{\bar{x}}_{i j}}\right)^{\frac{\varepsilon-1}{\varepsilon}}\right)^{\frac{\varepsilon}{\varepsilon-1}},
$$

where $x_{i j}$ are intermediate inputs from industry $j$ used by industry $i$.

\section{Data and Calibration}

We work with the 88 sector US KLEMS annual input-output data from Dale Jorgenson and his collaborators, dropping the government sectors. The dataset contains sectoral output and inputs from 1960 to 2005 . We use the sector-level TFP series computed by Carvalho and Gabaix (2013) using the methodology of Jorgenson et al. (1987).

We calibrate the expenditure share parameters to match the input-output table, using 1982 (the middle of the sample) as the base year. We specify sectoral TFP shocks to be lognormally distributed so that $\log A_{i} \sim \mathcal{N}\left(-\Sigma_{i i} / 2, \Sigma_{i i}\right)$, where $\Sigma_{i i}$ is the sample variance of $\log$ TFP growth for industry $i$. We work with uncorrelated sectoral shocks since the average correlation between sectoral growth rates is small (less than $5 \%$ ). Our results are not significantly affected if we matched the whole covariance matrix of sectoral TFP instead.

We consider shocks at annual and quadrennial horizons, the latter corresponding to the average period of a business cycle. The average standard deviation at a quadrennial frequency is about twice its value at an annual frequency. In fact, it is the only difference between the annual and quadrennial calibrations. Nonlinearities, which matter more for bigger shocks, are more important at a quadriennal frequency than at an annual frequency.

Our specification assumes only three distinct structural microeconomic elasticities of substitution. This is because estimates of more disaggregated elasticities are not available. For our benchmark calibration, we set $(\sigma, \theta, \varepsilon)=(0.9,0.5,0.001)$. We set the elasticity of substitution in consumption $\sigma=0.9$, following Atalay (2017), Herrendorf et al. (2013), and Oberfield and Raval (2014), all of whom use an elasticity of substitution in consumption (across industries) of slightly less than one. For the elasticity of substitution across valueadded and intermediate inputs, we set $\theta=0.5$. This accords with the estimates of Atalay (2017), who estimates this parameter to be between 0.4 and 0.8, as well as Boehm et al. (2017), who estimate this elasticity to be close to zero. Finally, we set the elasticity of substitution across intermediate inputs to be $\varepsilon=0.001$, which matches the estimates of Atalay (2017).

Owing to uncertainty surrounding the estimates for $(\sigma, \theta, \varepsilon)$, we include many robustness checks in Tables 4-7 in Appendix D. In the main text in Tables 1 and 2, we focus and magnify the nonlinearities of the model. 
on four sets of elasticities $(\sigma, \theta, \varepsilon)$ : our benchmark calibration $(0.9,0.5,0.001)$; a calibration with lower but still plausible elasticities $(0.7,0.3,0.001)$; a calibration with higher elasticities $(0.9,0.6,0.2)$; and a (close to) Cobb-Douglas calibration $(0.99,0.99,0.99)$. In Appendix $\mathrm{D}$, we report robustness checks for different values of these elasticities on a grid with $\sigma \in\{0.8,0.9,0.99\}, \theta \in\{0.4,0.5,0.6,0.99\}$, and $\varepsilon \in\{0.001,0.2,0.99\}$. Our results in Table 1 are not sensitive to the exact value of $(\sigma, \theta, \varepsilon)$ provided that the elasticities are collectively low enough that the calibration matches the observed volatility of the Domar weights.

Since the volatility of Domar weights are a measure of the size of the second-order terms in the model, we use the volatility of Domar weights as a sanity check for our calibration. Specifically, we target $\sigma_{\lambda}=\sum_{i} \bar{\lambda}_{i} \sigma_{\lambda_{i}}$, where $\bar{\lambda}_{i}$ is the time-series average of the $i$ th Domar weight and $\sigma_{\lambda_{i}}$ is the time-series standard deviation of industry $i^{\prime}$ s log-differenced Domar weight. In our data, at annual frequency, $\sigma_{\lambda} \approx 0.13$ and for quadrennial frequency $\sigma_{\lambda} \approx 0.27$. With no labor-reallocation, our baseline calibrations match these numbers relatively well; the lower-elasticity calibrations overshoot; the higher-elasticity calibrations undershoot; and the Cobb-Douglas calibrations deliver zero volatility of the Domar weights. Allowing for labor re-allocation reduces the volatility of the Domar weights. ${ }^{40}$

\section{Results}

Table 1 displays the mean, standard deviation, skewness, and excess kurtosis of log aggregate output for various specifications. For comparison, the table also shows these moments for aggregate TFP growth in the data. ${ }^{41}$ In addition, we also report the volatility of the Domar weights, both in the model and in the data. Since we have too few annual (and even fewer quadrennial) observations of aggregate TFP, we do not report the skewness and excess kurtosis: the implied confidence intervals for these estimates would be so large as to make the point estimates uninformative. In Appendix D, we report results for numerous other permutations of the elasticities of substitution for robustness.

Overall, given our elasticities of substitution, the model with full reallocation is unable to replicate the volatility of the Domar weights at either annual or quadrennial frequency, suggesting that this model is not nonlinear enough to match the movements in the Domar weights as arising from sectoral productivity shocks. On the other hand, the model without

\footnotetext{
${ }^{40}$ To match the data, we assume that changes in sectoral TFPs are the sole driver of fluctuations in the Domar weight. Other types of shocks, besides industry-level TFP shocks, could also drive volatility to the Domar weights (for example shocks to the composition of demand). While such shocks, whatever they are, would also indicate the presence nonlinearities, they would imply different elasticities of substitution for the calibration of the micro-elasticities of substitution. We abstract from this issue in calibrating the parameters of the model in Section 6.1.

${ }^{41}$ Since our model has inelastic factor supply, its output is more comparable to aggregate TFP than to real GDP. As shown by Gabaix (2011) and Carvalho and Gabaix (2013), elastic capital and labor supply would further amplify TFP shocks.
} 


\begin{tabular}{llllll}
$(\sigma, \theta, \epsilon)$ & Mean & Std & Skew & Ex-Kurtosis & $\sigma_{\lambda}$ \\
\hline \hline
\end{tabular}

Full Reallocation - Annual

$\begin{array}{lrrrrr}(0.7,0.3,0.001) & -0.0023 & 0.011 & -0.10 & 0.1 & 0.090 \\ (0.9,0.5,0.001) & -0.0022 & 0.011 & -0.08 & 0.0 & 0.069 \\ (0.9,0.6,0.2) & -0.0020 & 0.011 & -0.05 & 0.0 & 0.056 \\ (0.99,0.99,0.99) & -0.0013 & 0.011 & 0.01 & 0.0 & 0.001\end{array}$

No Reallocation - Annual

$\begin{array}{lrrrrr}(0.7,0.3,0.001) & -0.0045 & 0.012 & -0.31 & 0.4 & 0.171 \\ (0.9,0.5,0.001) & -0.0034 & 0.012 & -0.18 & 0.1 & 0.115 \\ (0.9,0.6,0.2) & -0.0024 & 0.011 & -0.11 & 0.1 & 0.068 \\ (0.99,0.99,0.99) & -0.0011 & 0.011 & 0.00 & 0.0 & 0.001\end{array}$

Full Reallocation - Quadrennial

$\begin{array}{lrrrrr}(0.7,0.3,0.001) & -0.0118 & 0.026 & -0.4 & 0.4 & 0.307 \\ (0.9,0.5,0.001) & -0.0113 & 0.026 & -0.28 & 0.4 & 0.176 \\ (0.9,0.6,0.2) & -0.0100 & 0.026 & -0.23 & 0.2 & 0.133 \\ (0.99,0.99,0.99) & -0.0058 & 0.025 & 0.01 & 0.0 & 0.003\end{array}$

No Reallocation - Quadrennial

$\begin{array}{lrrrrr}(0.7,0.3,0.001) & -0.0270 & 0.037 & -2.18 & 12.7 & 0.404 \\ (0.9,0.5,0.001) & -0.0187 & 0.030 & -1.11 & 3.6 & 0.267 \\ (0.9,0.6,0.2) & -0.0129 & 0.027 & -0.44 & 0.7 & 0.154 \\ (0.99,0.99,0.99) & -0.0057 & 0.025 & 0.00 & 0.0 & 0.002\end{array}$

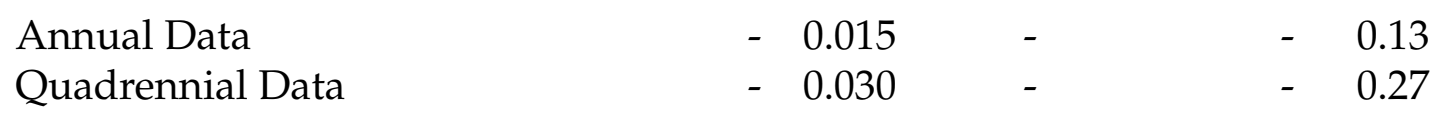

Table 1: Simulated and estimated moments. For the data, we use the demeaned growth rate of aggregate TFP. For the model, we use the sample moments of log output. The simulated moments are calculated from 50,000 draws. Skewness and kurtosis of the data are impossible to estimate with enough precision and so we do not report them. 
reallocation is able to match the volatility of the Domar weights, which is consistent with the intuition of Section 3.

Let us consider each moment in turn, starting with the mean. For our benchmark calibration $(\sigma, \theta, \varepsilon)=(0.9,0.5,0.001)$, the model without reallocation matches the volatility of Domar weights at both annual and quadrennial frequency. The reductions in the mean are around $0.3 \%$ at annual and just under $2 \%$ at quadrennial frequency. Since our lognormal shocks are calibrated to have a mean of 1 in levels, as we increase the variance, the mean of the log declines. We can net out this mechanical effect by subtracting the average loss in performance from the ones in the loglinear Cobb-Douglas model. At an annual frequency, this results in a loss from nonlinearities of $0.34 \%-0.11 \%=0.23 \%$, and at a quadrennial frequency, the loss from nonlinearities is $1.87 \%-0.57 \%=1.30 \%$. These numbers, which identify the welfare costs of sectoral shocks arising from concavity in production, can be compared with the welfare costs of fluctuations à la Lucas (1987) arising from concavity in utility, which are around $0.01 \%$ and $0.05 \%$ respectively in these calibrations. The reduction in the mean increases as we pump up the degree of nonlinearity, and so do the corresponding losses from nonlinearities.

A qualitatively similar pattern holds for the model with full reallocation. In that case, the reductions in the mean are smaller: $0.2 \%-0.1 \%=0.1 \%$ at an annual frequency and $1.1 \%-0.57 \%=0.46 \%$ at a quadrennial frequency. Unsurprisingly, the approximately CobbDouglas model behaves similarly regardless of the mobility of labor - this follows from the fact that Hulten's theorem holds globally for a Cobb-Douglas model where no labor reallocation takes place in equilibrium whether or not it is allowed.

At an annual frequency, both models undershoot a bit on the overall volatility of aggregate TFP. One reason why the model undershoots on standard deviation, particularly at annual frequency, is that we restrict the industry-level productivity shocks to be independent, whereas in the data, particularly at higher frequencies, they feature some correlation. At a quadrennial frequency, the model better matches the volatility of aggregate TFP. The degree of nonlinearity makes little difference for the volatility of aggregate TFP at an annual frequency. ${ }^{42}$ Nonlinearities matter more for the volatility of aggregate TFP at a quadrennial frequency because the shocks are larger.

Finally, the models with and without reallocation both generate negative skewness and some positive excessive kurtosis (in fact, a very high amount for the quadrennial specifications without reallocation). The skewness and excess kurtosis fatten the left tail of the

\footnotetext{
${ }^{42}$ Whereas for the mean, skewness, and kurtosis, the second-order terms are the dominant power in the Taylor expansion (since the linear terms have no effect), for the variance, the dominant power is the linear term. For example, letting $\sigma$ be the standard deviation of the shocks, the approximation of the variance in Appendix E shows that the contribution of the linear terms scales in $\sigma^{2}$, whereas that of the nonlinear terms scales in $\sigma^{4}$.
} 
distribution, providing an endogenous explanation for "rare disasters". Unlike Acemoglu et al. (2017) or Barro (2006), to achieve rare disasters, we do not need to assume fat-tailed exogenous shocks nor rule out "rare bonanzas" a priori. Instead these features are endogenously generated by the nonlinearities in the model. This can be seen in Figure 5, where we plot the histograms for the benchmark calibrations with no reallocation (which match the volatility of Domar weights) and for a loglinear approximation subject to the same shocks. As expected, nonlinearities are more important at a quadrennial frequency than at an annual frequency because the shocks are larger (more volatile).
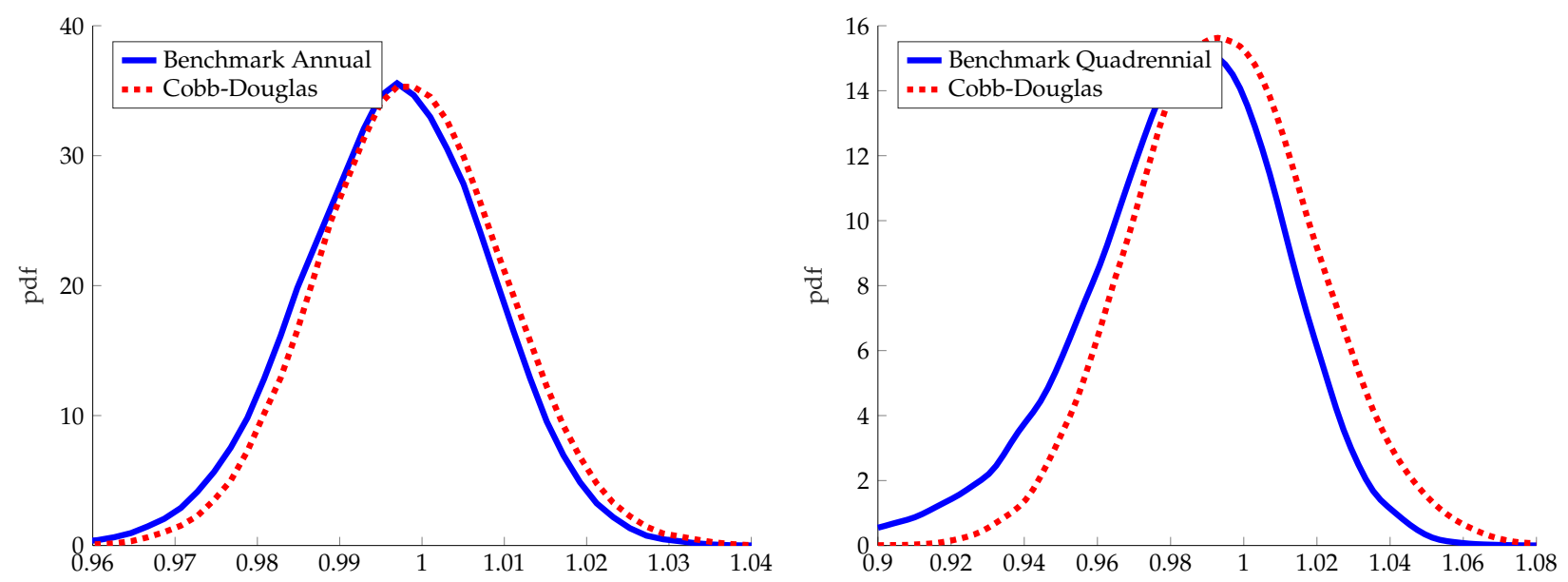

Figure 5: The left panel shows the distribution of aggregate output for the benchmark model and loglinear model at an annual frequency. The right panel shows these for shocks at a quadrennial frequency. The difference between the two frequencies is that shocks are larger (their volatility is higher) at a quadrennial than at an annual frequency. The benchmark model has $(\sigma, \theta, \varepsilon)=(0.9,0.5,0.001)$ and no labor reallocation. Note that the scales are different in these two figures.

We also consider the response of aggregate output to shocks to specific industries, using our benchmark calibration. It turns out that for a large negative shock, the "oil and gas" industry produces the largest negative response in aggregate output, despite the fact it is not the largest industry in the economy. Figure 6 plots the response of aggregate output for shocks to the oil and gas industry as well as for the "retail trade (excluding automobiles)" industry. The retail trade industry has a similar sales share, and therefore, to a first-order, both industries are equally important. As expected, the nonlinear model amplifies negative shocks and mitigates positive shocks. However, whereas output is roughly loglinear for shocks to retail trade, output is highly nonlinear with respect to shocks to oil and gas.

The intuition for this asymmetry comes from the examples in Figures 3 and 4. "Oil and ga's" is an approximately universal input, so that the downstream elasticity of substitution (in consumption) $\sigma$ is less relevant and the upstream elasticity of substitution (in production) 
$\theta$ is more relevant. Since $\theta \ll \sigma$ in our calibration, this means that output is more nonlinear in shocks to "oil and gas" than in shocks to "retail trade". Furthermore, "oil and gas" have a relatively low share of intermediate input usage. As a result, in response to a negative shock, resources cannot be reallocated to boost the production of oil and gas. This means that we are closer to the economy depicted in Figure 4 than the one in Figure 3, with a correspondingly lower GE elasticity of substitution between oil and gas and everything else due to the lack of reallocation.

The strong asymmetry between the effects of positive and negative shocks is consistent with the empirical findings of Hamilton (2003) that oil price increases are much more important than oil price decreases. ${ }^{43}$

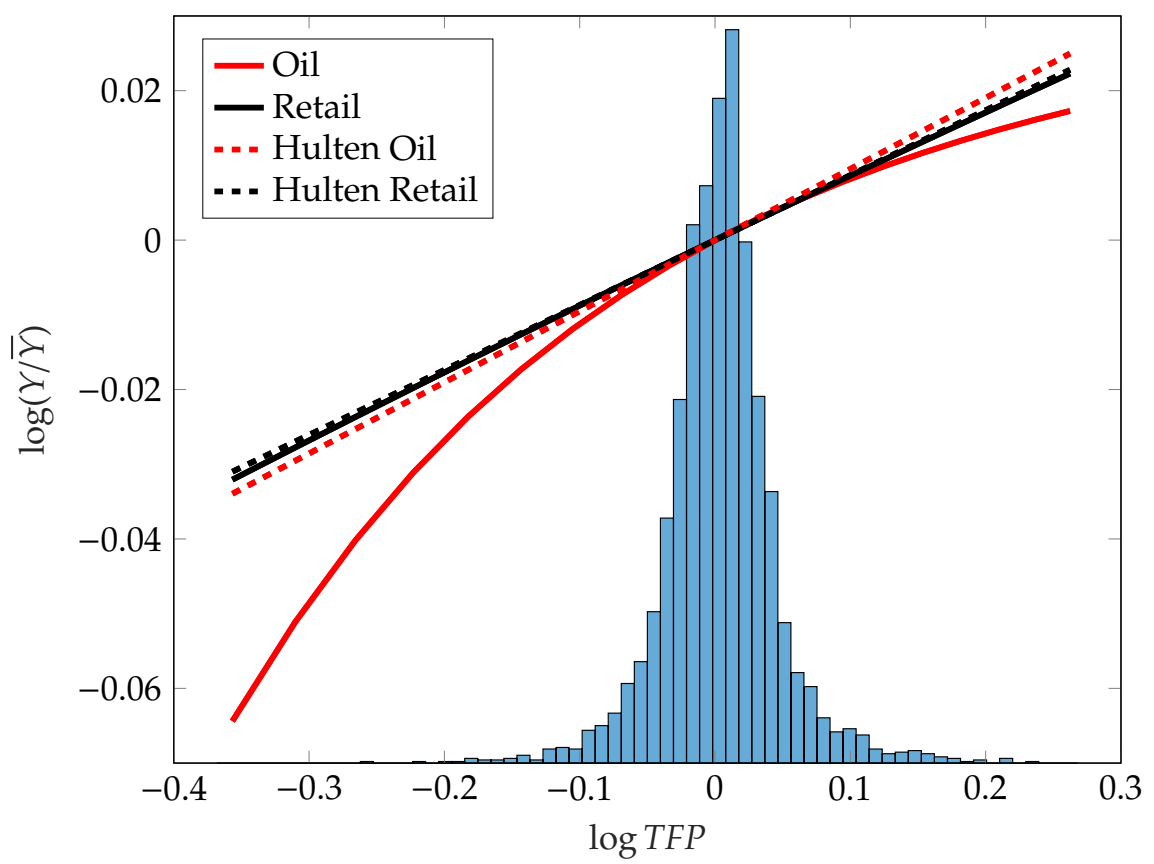

Figure 6: The effect of TFP shocks to the oil and gas industry and the retail trade industry. Both industries have roughly the same sales share, and so they are equally important up to a first-order approximation (dotted line). The nonlinear model is more fragile to both shocks than the loglinear approximation. The "oil and gas" industry is significantly more important than "retail trade" for large negative shocks. The histogram is the empirical distribution of sectoral annual TFP shocks pooled over the whole sample. The model has $(\sigma, \theta, \varepsilon)=(0.9,0.5,0.001)$ with no labor reallocation and no adjustment costs.

\footnotetext{
${ }^{43}$ Figure 6 may give the impression that the relative ranking of industries is stable as a function of the size of the shock. The oil industry is always more important than the retail trade industry for negative shocks, and always less important for positive shocks. However, this need not be the case. In Appendix D we plot aggregate output as a function of shocks to the "oil and gas" industry and the construction industry. The construction industry is larger than the oil industry. Therefore, the first-order approximation implies that it should be more important. The nonlinear model also behaves the same way for positive shocks, and small negative shocks. However, for very large negative shocks, the "oil and gas" industry once again becomes more important.
} 


\subsection{The Effect of Oil Shocks}

In this section, we use the oil shocks of the 1970s to demonstrate the way nonlinearities can amplify the macroeconomic impact of industry-level shocks. ${ }^{44}$ To recap the history, starting in 1973, coordinated action by OPEC caused the price of crude oil to increase from $\$ 3.5$ per barrel in 1972 to $\$ 11$ per barrel in 1974. In 1979, OPEC implemented a second round of quantity restrictions which caused the price of crude to soar to $\$ 31$ per barrel. Shortly after this, the Iranian revolution of 1979 and the ensuing Iraqi invasion of Iran caused further disruptions to global crude oil supply. The price peaked at \$37 in 1980. Starting in the early 1980s, with the abdication of the Shah, OPEC's pricing structure collapsed and, in a bid to maintain its market share, Saudi Arabia flooded the market with inexpensive oil. In real terms, the price of crude oil declined back to its pre-crisis levels by 1986.

We adopt a non-parametric approach which allows us to account for the second-order macroeconomic impact of microeconomic shocks using ex-post data. Instead of trying to predict how Domar weights change in response to a shock as would be required for a counterfactual exercise, we can simply observe it. Formally, we rely on the following result.

Proposition 10. Up to the second order in the vector $\Delta$, we have

$$
\log (Y(A+\Delta) / Y(A))=\frac{1}{2}[\lambda(A+\Delta)+\lambda(A)]^{\prime}(\log (A+\Delta)-\log (A))
$$

The idea of averaging weights across two periods is due to Törnqvist (1936). Proposition 10 relates the macroeconomic impact of microeconomic shocks to the size of the shock and the corresponding Domar weights before and after the shock. ${ }^{45}$

\footnotetext{
${ }^{44}$ Although our structural model suggests that the "oil and gas" extraction industry is important, it abstracts away from trade, by assuming all intermediate inputs are sourced domestically, with net imports showing up only in final demand. Hence, the Domar weight of the "oil and gas" industry measures domestic production, rather than domestic consumption. Since the oil price shocks did not directly affect the productivity of domestic oil production, this means that they are not measured in our sectoral TFP data (which is for domestic production). Furthermore, our industry classification is too coarse to isolate crude oil separately from other petrochemicals. For this reason, we use global (rather than US) data.

${ }^{45}$ One can always compute the full nonlinear impact of a shock on output by computing $\int_{A}^{A+\Delta} \lambda(\tilde{A}) \mathrm{d} \log \tilde{A}$, and our formula approximates this integral by performing a first-order (log) approximation of the Domar weight $\lambda(\tilde{A})$ or equivalently a second-order (translog) approximation of aggregate output. In theory, if TFP is a continuous diffusion then one can disaggregate time-periods and compute the impact of shocks over a time period $[t, t+\delta]$ as $\int_{t}^{t+\delta} \lambda\left(A_{s}\right) \mathrm{d} \log \left(A_{s}\right)$ which can be seen as a repeated application of Hulten's theorem at every point in time over infinitesimal intervals of time. However, when TFP has jumps, then this decomposition no longer applies. In any case, even when it does apply, and when the required high-frequency data regarding TFP shocks and Domar weights is available, it can only be useful ex post to asses the changes in aggregate output over an elapsed period of time due to the TFP shocks $\mathrm{d} \log \left(A_{s}\right)$ to a given sector given the observed path of Domar weight $\lambda\left(A_{s}\right)$. It is of no use ex ante to predict how these future shocks will affect aggregate output because one would need to know how the Domar weight will change over time as a result of the shocks, and hence of no use to run counterfactuals. This latter part is precisely what the second-order approximation at the heart of our paper accomplishes.
} 
We measure the price of oil using the West Texas Intermediate Spot Crude Oil price from the Federal Reserve Database. Global crude oil production, measured in thousand tonne of oil equivalents, is from the OECD. World GDP, in current USD, is from the World Bank national accounts data. The choice of the pre and post Domar weight is not especially controversial. Crude oil, as a fraction of world GDP, increased from 1.8\% in 1972 to $7.6 \%$ in 1979. Reassuringly, the Domar weight is back down to its pre-crisis level by 1986 (see Figure 7). This means that, taking the second-order terms into account, we need to weight the shock to the oil industry by $1 / 2(1.8 \%+7.6 \%)=4.7 \%$. Hence, the second-order terms amplify the shock by a factor of $4.7 / 1.8 \approx 2.6$.

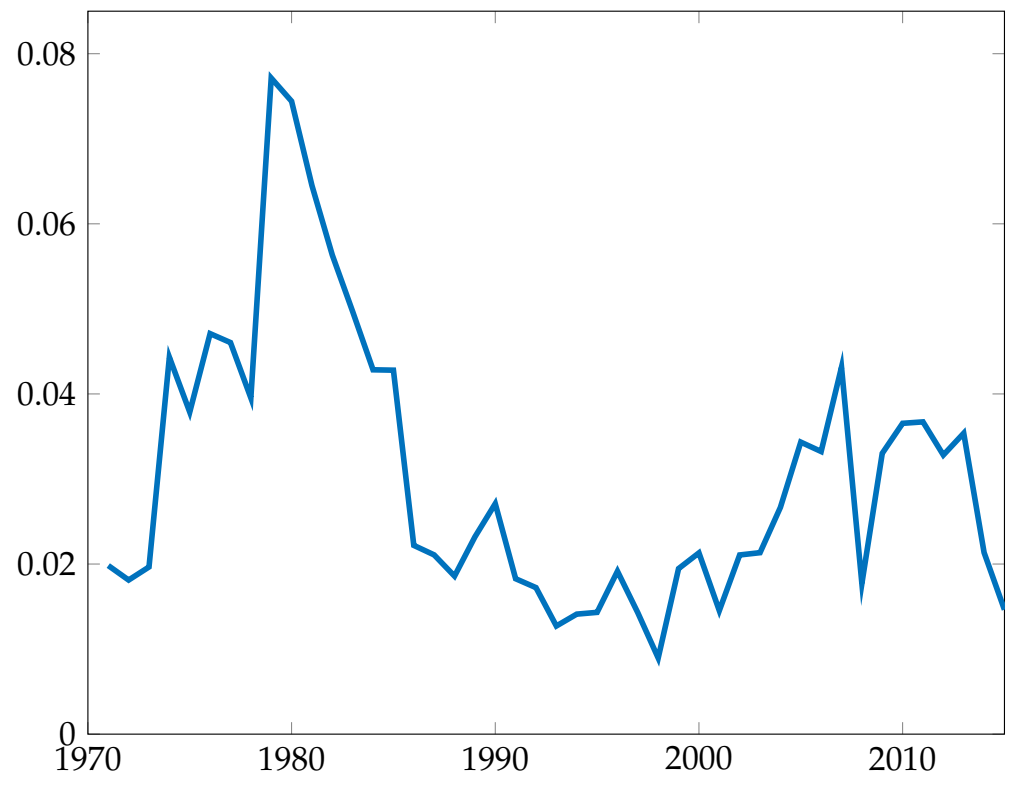

Figure 7: Global expenditures on crude oil as a fraction of world GDP.

Calibrating the size of the shock to the oil industry is more tricky, since it is not directly observed. If we assume that oil is an endowment, then we can simply measure the shock via changes in the physical quantity of production. To do this, we demean the log growth rate in global crude oil production, and take the shock to be the cumulative change in demeaned growth rates from 1973 to 1980 , which gives us a shock of $-13 \% .{ }^{46}$

Putting this altogether, the first-order impact on aggregate output is therefore

$$
1.8 \% \times-13 \%=-.23 \%
$$

\footnotetext{
${ }^{46}$ We use the demeaned growth rate to remove the overall (positive) trend in production. Intuitively, if everything is growing at the same rate, then a negative oil shock is a reduction in the growth of oil relative to trend. Of course, one can easily quibble with this estimate of the size of the shock, but fortunately, the degree of amplification (defined as the ratio of the second-order approximation to the first) is independent of our estimate for the size of the shock. So, for any value of the shock, the second-order approximation almost triples the impact of the shock.
} 
On the other hand, the second-order impact on aggregate output is

$$
\frac{1}{2}(1.8 \%+7.6 \%) \times-13 \%=-.61 \% \text {. }
$$

Hence, accounting for the second-order terms amplifies the impact of the oil shocks significantly, so that oil shocks can be macroeconomically significant even without any financial or demand side frictions. ${ }^{47}$

\subsection{Baumol's Cost Disease and Long-Run Growth}

Our final empirical exercise looks to quantify the importance of nonlinearities on long-run productivity growth. For this exercise, we use World KLEMS data for the US from 1948-2014.

The "nonlinear" measure of aggregate TFP growth over the sample is built by updating the Domar weights every period:

$$
\Delta \log T F P^{\text {nonlinear }}=\sum_{i=1}^{N} \sum_{t=1948}^{2013} \lambda_{i, t}\left(\log A_{i, t+1}-\log \left(A_{i, t}\right)\right) .
$$

This provides an approximation, by discrete left Riemann sums, of the exact aggregate TFP growth, given by the sum of continuous integrals $\sum_{i=1}^{N} \int_{1948}^{2014} \lambda_{i, t} d \log A_{i, t}$.

Now consider the following counterfactual: imagine that the economy is loglinear so that the Domar weights are constant throughout the sample at their 1948 values. Assuming that the path for industry-level TFP is unchanged, aggregate TFP growth over the sample would be given by

$$
\Delta \log \text { TFP }^{1 \text { st order }}=\sum_{i=1}^{N} \lambda_{i, 1948}\left(\log A_{i, 2014}-\log A_{i, 1948}\right)
$$

By comparing actual aggregate TFP growth and TFP growth in the counterfactual loglinear economy, we quantify the importance of Baumol's cost disease (Baumol, 1967): the notion that over time, because of complementarities, the sales shares of low-productivitygrowth industries increase while those of high-productivity-growth industries decrease,

\footnotetext{
${ }^{47}$ As noted by Hamilton (2013), first-order approximations of efficient models assign a relatively small impact to oil price shocks. Hence, the literature has tended to focus on various frictions that may account for the strong statistical relationship between oil shocks and aggregate output. Our calculations suggests that nonlinearities in production may help explain the outsized effect of oil shocks even in efficient models. Furthermore, our calculation makes no allowance for amplification of shocks through endogenous labor supply and capital accumulation, which are the standard channels for amplification of shocks in the business cycle literature. Hence, coupled with the standard amplification mechanisms of those models, we would expect the reduction in aggregate output to be even larger.
} 
thereby slowing down aggregate TFP growth.

By the end of the sample, aggregate TFP growth in the counterfactual loglinear or CobbDouglas economy is around $87 \%$ whereas actual TFP grew by $68 \%$. Hence the presence of nonlinearities slowed down aggregate TFP growth by around 19 percentage points over the sample period.

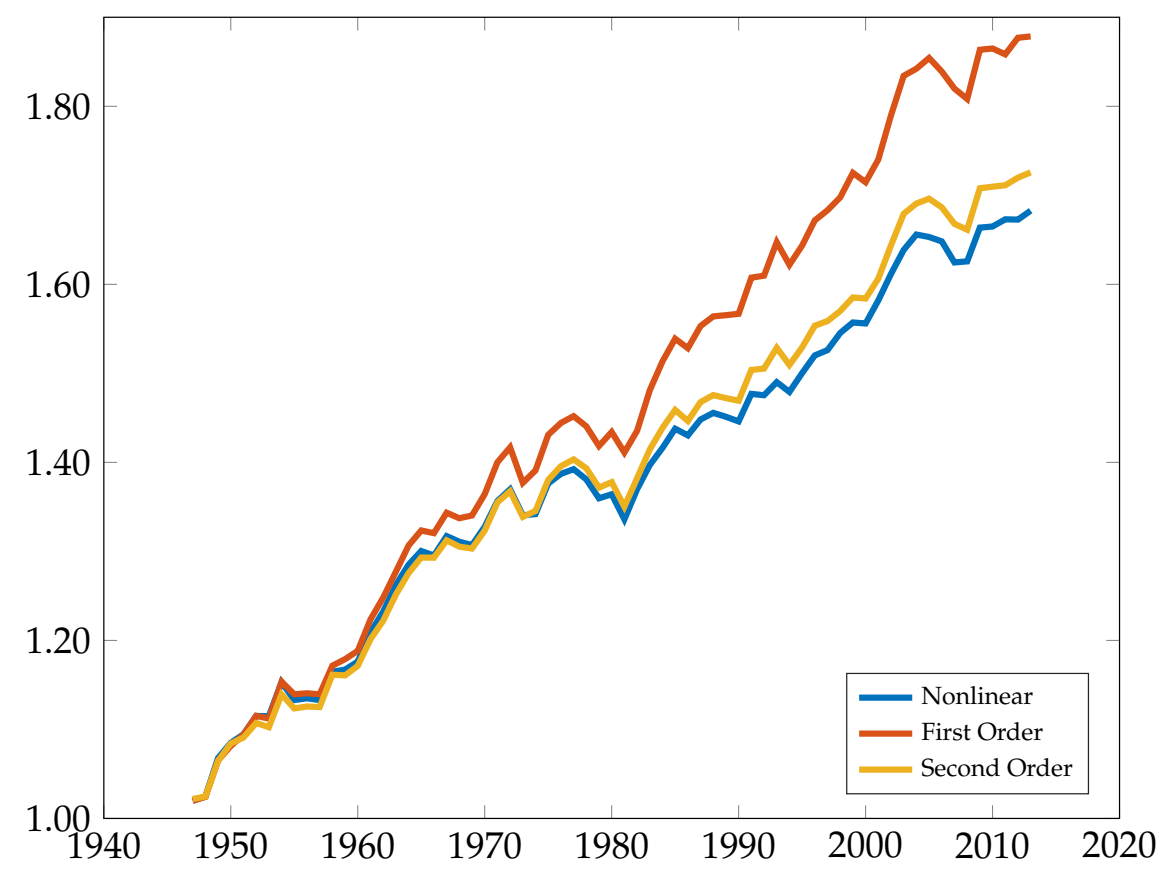

Figure 8: Cumulative change in TFP: nonlinear (actual), first-order approximation, and second-order approximation.

Baumol's cost disease is a manifestation of nonlinearities, since $\Delta \log T F P^{1 s t}$ order is a firstorder approximation of actual aggregate TFP growth. A second-order approximation, which captures some of the nonlinearities, is given by

$$
\Delta \log T F P^{2 \text { nd order }}=\frac{1}{2} \sum_{i=1}^{N}\left(\lambda_{i, 1948}+\lambda_{i, 2014}\right)\left(\log A_{i, 2014}-\log A_{i, 1948}\right) .
$$

In Figure 8, we plot aggregate TFP growth using the nonlinear, first-order, and secondorder measures. The gap between the nonlinear measure and the first-order approximation is sizeable, but that between the nonlinear measure and the second-order approximation is much smaller.

These findings are in some sense more general than Baumol's hypothesized mechanism. Traditionally, the mechanism for Baumol's cost disease is through prices: because of complementarities, as the prices of high-productivity-growth industries fall, their shares in aggregate output fall, resources are reallocated away from them, thereby reducing ag- 
gregate TFP growth. Alternative stories for structural transformation emphasize other mechanisms, principally, non-homotheticities whereby as real income increases, consumers increase their expenditures on industries that happen to have lower productivity growth. ${ }^{48}$ From the perspective of our framework, non-homotheticities and complementarities are both nonlinearities. Our calculations of the impact of nonlinearities encompass both types of mechanisms. ${ }^{49,50}$

\section{Conclusion}

The paper points to many unanswered questions. For instance, it shows that the macroeconomic impact of a microeconomic shock depends greatly on how quickly factors can be reallocated across production units. Since our structural model is static, we are forced to proxy for the temporal dimension of reallocation by resorting to successive comparative statics. In ongoing work, we investigate the dynamic adjustment process more rigorously and find that although we can think of the no-reallocation and perfect-reallocation cases as the beginning and end of the adjustment, the speed of adjustment also greatly depends on the microeconomic details. This means that the dynamic response of aggregate output to different shocks is greatly affected by issues like geographic or sectoral mobility of labor, even with perfect and complete markets that allow us to abstract from distributional issues. Our structural application also lacks capital accumulation and endogenous labor supply, and incorporating these into the present analysis is an interesting area for future work.

Furthermore, in this paper, we have focused exclusively on the way shocks affect aggregate output. In Baqaee and Farhi (2018a), we focus on how shocks affect factor shares and derive characterizations of the macroeconomic elasticities of substitution between factors and of the macroeconomic bias of technical change. In Baqaee and Farhi (2018b), we take up the related question of how shocks affect non-aggregate outcomes, namely how shocks propagate from one producer to another, and how microeconomic variables comove with one another in a production network.

In addition, this paper assumes that aggregate final demand is homothetic. Nonhomoth-

\footnotetext{
${ }^{48}$ See Herrendorf et al. (2013) as an example.

${ }^{49}$ Our theoretical characterizations cover all these nonlinearities. In fact, formally, non-homotheticities can always be represented via non-unitary elasticities of substitution between inputs and a fixed factor. To see this, note that any non-homothetic function $f(x)$ can be extended into a constant-returns function $\tilde{f}(x, y)=y f(x / y)$ where $f(x)=\tilde{f}(x, 1)$. Then, non-homotheticity in $f$ is equivalent to a non-unitary elasticity of substitution between $x$ and $y$ in $\tilde{f}(x, y)$.

${ }^{50}$ Another potential source of nonlinearities is shocks to the composition of demand. For example, a CobbDouglas model with shocks to the share parameters is a nonlinear model since the cross-partial derivatives of aggregate output with respect to industry TFP and share shocks are non-zero. Our results also cover such economies.
} 
etic aggregate final demand can arise from nonhomothetic individual final demand or from the aggregation of heterogenous but homothetic individual demands. In Baqaee and Farhi (2018b, 2019), we show how to take these elements into account. In particular, in Baqaee and Farhi (2018b), we show how to combine input-output production networks with heteorgeneous agents and non-homothetic final demand in closed economies. In Baqaee and Farhi (2019), we show how to take these elements into account in trade models.

Finally, this paper assumes away non-technological frictions, but the forces we identify do not disappear in richer models with inefficient equilibria. Non-unitary elasticities of substitution in networks can amplify or attenuate the underlying distortions. In Baqaee and Farhi (2018c), we undertake a systematic characterization of these effects. We show that in inefficient models, the "second-order" terms that we characterize in this paper can become first order.

\section{References}

Acemoglu, D., U. Akcigit, and W. Kerr (2015). Networks and the macroeconomy: An empirical exploration. NBER Macro Conference.

Acemoglu, D., D. Autor, D. Dorn, G. H. Hanson, and B. Price (2016). Import competition and the great US employment sag of the 2000s. Journal of Labor Economics 34(S1), S141-S198.

Acemoglu, D., V. M. Carvalho, A. Ozdaglar, and A. Tahbaz-Salehi (2012). The network origins of aggregate fluctuations. Econometrica 80(5), 1977-2016.

Acemoglu, D., A. Ozdaglar, and A. Tahbaz-Salehi (2015). Systemic risk and stability in financial networks. The American Economic Review 105(2), 564-608.

Acemoglu, D., A. Ozdaglar, and A. Tahbaz-Salehi (2016). Networks, shocks, and systemic risk. In The Oxford Handbook of the Economics of Networks.

Acemoglu, D., A. Ozdaglar, and A. Tahbaz-Salehi (2017). Microeconomic origins of macroeconomic tail risks. The American Economic Review 107(1), 54-108.

Aghion, P. and P. W. Howitt (2008). The economics of growth. MIT press.

Altinoglu, L. (2016). The origins of aggregate fluctuations in a credit network economy.

Arkolakis, C., A. Costinot, and A. Rodríguez-Clare (2012). New trade models, same old gains? American Economic Review 102(1), 94-130.

Atalay, E. (2017). How important are sectoral shocks? American Economic Journal: Macroeconomics 9(4), $254-80$.

Autor, D. H., D. Dorn, and G. H. Hanson (2016). The china shock: Learning from labor-market adjustment to large changes in trade. Annual Review of Economics 8, 205-240.

Ballester, C., A. Calvó-Armengol, and Y. Zenou (2006). Who's who in networks. wanted: the key player. Econometrica 74(5), 1403-1417.

Baqaee, D. and E. Farhi (2018a). The microeconomic foundations of aggregate production functions. Technical report, National Bureau of Economic Research.

Baqaee, D. R. (2015). Targeted fiscal policy.

Baqaee, D. R. (2018). Cascading failures in production networks. Econometrica 86(5), 1819-1838. 
Baqaee, D. R. and E. Farhi (2018b). Macroeconomics with heterogeneous agents and input-output networks. Technical report, NBER Working Papers 24684, National Bureau of Economic Research, Inc.

Baqaee, D. R. and E. Farhi (2018c). Productivity and misallocation in general equilibrium. Technical report, National Bureau of Economic Research.

Baqaee, D. R. and E. Farhi (2019). Trade theory with global production networks. Technical report.

Barro, R. J. (2006). Rare disasters and asset markets in the twentieth century. The Quarterly Journal of Economics, $823-866$.

Barrot, J.-N. and J. Sauvagnat (2016). Input specificity and the propagation of idiosyncratic shocks in production networks. The Quarterly Journal of Economics.

Bartelme, D. and Y. Gorodnichenko (2015). Linkages and economic development. Technical report, National Bureau of Economic Research.

Baumol, W. J. (1967). Macroeconomics of unbalanced growth: the anatomy of urban crisis. The American economic review 57(3), 415-426.

Bigio, S. and J. La'O (2016). Financial frictions in production networks. Technical report.

Blackorby, C. and R. R. Russell (1989). Will the real elasticity of substitution please stand up?(a comparison of the Allen/Uzawa and Morishima elasticities). The American economic review 79(4), 882-888.

Boehm, C. E., A. Flaaen, and N. Pandalai-Nayar (2017). Input linkages and the transmission of shocks: Firm-level evidence from the 2011 tohoku earthquake. The Review of Economics and Statistics.

Burstein, A. and J. Cravino (2015). Measured aggregate gains from international trade. American Economic Journal: Macroeconomics 7(2), 181-218.

Carvalho, V. and X. Gabaix (2013). The great diversification and its undoing. The American Economic Review 103(5), 1697-1727.

Carvalho, V. M. (2010). Aggregate fluctuations and the network structure of intersectoral trade.

Costinot, A. and A. Rodriguez-Clare (2014). Trade theory with numbers: quantifying the consequences of globalization. Handbook of International Economics 4, 197.

Di Giovanni, J., A. A. Levchenko, and I. Méjean (2014). Firms, destinations, and aggregate fluctuations. Econometrica 82(4), 1303-1340.

Durlauf, S. N. (1993). Nonergodic economic growth. The Review of Economic Studies 60(2), 349-366.

Elliott, M., B. Golub, M. O. Jackson, et al. (2014). Financial networks and contagion. American Economic Review 104(10), 3115-53.

Feenstra, R. C. and G. H. Hanson (1996). Globalization, outsourcing, and wage inequality. The American Economic Review 86(2), 240.

Foerster, A. T., P.-D. G. Sarte, and M. W. Watson (2011). Sectoral versus aggregate shocks: A structural factor analysis of industrial production. Journal of Political Economy 119(1), 1-38.

Gabaix, X. (2011). The granular origins of aggregate fluctuations. Econometrica 79(3), 733-772.

Gabaix, X. (2016). Power laws in economics: An introduction. Journal of Economic Perspectives 30(1), 185-206.

Gomme, P. and P. Rupert (2007). Theory, measurement and calibration of macroeconomic models. Journal of Monetary Economics 54(2), 460-497.

Grassi, B. (2017). IO in I-O: Competition and volatility in input-output networks. Technical report.

Grossman, G. M., E. Helpman, E. Oberfield, and T. Sampson (2016). Balanced growth despite Uzawa. Technical report, National Bureau of Economic Research.

Hamilton, J. D. (2003). What is an oil shock? Journal of econometrics 113(2), 363-398.

Hamilton, J. D. (2013). Historical oil shocks. In R. E. Parker and R. M. Whaples (Eds.), Routledge Handbook of Major Events in Economic History, Chapter 21, pp. 239-265. Routledge. 
Herrendorf, B., R. Rogerson, and A. Valentinyi (2013). Two perspectives on preferences and structural transformation. American Economic Review 103(7), 2752-89.

Hicks, J. (1932). Theory of wages. Springer.

Horvath, M. (1998). Cyclicality and sectoral linkages: Aggregate fluctuations from independent sectoral shocks. Review of Economic Dynamics 1(4), 781-808.

Horvath, M. (2000). Sectoral shocks and aggregate fluctuations. Journal of Monetary Economics 45(1), 69-106.

Hulten, C. R. (1978). Growth accounting with intermediate inputs. The Review of Economic Studies, 511-518.

Jones, C. I. (2011). Intermediate goods and weak links in the theory of economic development. American Economic Journal: Macroeconomics, 1-28.

Jones, C. I. (2013). Input-Output economics. In Advances in Economics and Econometrics: Tenth World Congress, Volume 2, pp. 419. Cambridge University Press.

Jorgenson, D. W., F. Gollop, and B. M Fraumeni (1987). Productivity and US economic growth.

Jovanovic, B. (1987). Micro shocks and aggregate risk. The Quarterly Journal of Economics, 395-409.

Kim, S.-J., H. S. Shin, et al. (2013). Working capital, trade and macro fluctuations. Technical report.

Liu, E. (2017). Industrial policies and economic development. Technical report.

Long, J. B. and C. I. Plosser (1983). Real business cycles. The Journal of Political Economy, 39-69.

Lucas, R. E. (1987). Models of business cycles, Volume 26. Basil Blackwell Oxford.

Morishima, M. (1967). A few suggestions on the theory of elasticity. Keizai Hyoron (Economic Review) 16, 144-150.

Notowidigdo, M. J. (2011). The incidence of local labor demand shocks. Technical report, National Bureau of Economic Research.

Oberfield, E. and D. Raval (2014). Micro data and macro technology. Technical report, National Bureau of Economic Research.

Scheinkman, J. A. and M. Woodford (1994). Self-organized criticality and economic fluctuations. The American Economic Review, 417-421.

Stella, A. (2015). Firm dynamics and the origins of aggregate fluctuations. Journal of Economic Dynamics and Control 55, 71-88.

Theil, H. (1967). Economics and information theory. Technical report.

Törnqvist, L. (1936). The Bank of Finland's consumption price index. Bank of Finland Monthly Bulletin 10. 


\section{A Proofs}

Proof of theorem 1. Since the first welfare theorem holds, the equilibrium allocation solves

$Y\left(A_{1}, \ldots, A_{N}\right)=\max _{c_{i}, x_{i j}, l_{i j}} \mathcal{D}\left(c_{1}, \ldots, c_{n}\right)+\sum_{i} \mu_{i}\left(A_{i} F_{i}\left(\left(l_{i j}\right)_{j},\left(x_{i j}\right)_{j}\right)-\sum_{j} x_{i j}-c_{i}\right)+\sum_{i} \lambda_{i}\left(\bar{l}_{i}-\sum_{j} l_{j i}\right)$,

where $\bar{l}_{i}$ is the endowment of each labor type, and $\mu_{i}$ and $\lambda_{i}$ are Lagrange multipliers. The envelope theorem then implies that

$$
\frac{\mathrm{d} Y}{\mathrm{~d} A_{i}}=\mu_{i} F_{i}\left(\left(l_{i j}\right)_{j},\left(x_{i j}\right)_{j}\right)=\mu_{i} y_{i} .
$$

If we show that $\mu_{i}$ is equal to the price of $i$ in the competitive equilibrium, then we are done.

Meanwhile, for each good $j$, either there exists another producer $i$ using that good as an input, or the household must consume that input (otherwise, the input is irrelevant and has a price of zero). Hence, in a competitive equilibrium, we must have either

$$
p_{i} \frac{\partial F_{i}}{\partial x_{i j}}=p_{j}
$$

and/or

$$
P_{c} \frac{\partial \mathcal{D}}{\partial c_{j}}=p_{j}
$$

where $P_{c}$ is the ideal price index associated with $\mathcal{D}$ (which we can take to be the numeraire). The expression above uses the fact that $\mathcal{D}$ is constant-returns-to-scale.

On the other hand, the first-order conditions of the social planners problem implies that for each $j$, either

$$
\mu_{i} \frac{\partial F_{i}}{\partial x_{i j}}=\mu_{j}
$$

and/or

$$
\frac{\partial \mathcal{D}}{\partial c_{j}}=\mu_{j}
$$

Hence $\mu_{i}=p_{i}$ for every $i$.

Proof of Theorem 2. Differentiate $\sum_{i} \lambda_{i}=\xi$ to get

$$
\lambda_{i} \frac{\mathrm{d} \log \lambda_{i}}{\mathrm{~d} \log A_{i}}=\xi \frac{\mathrm{d} \log \xi}{\mathrm{d} \log A_{i}}-\sum_{j \neq i} \lambda_{j} \frac{\mathrm{d} \log \lambda_{j}}{\mathrm{~d} \log A_{i}}
$$




$$
=\xi \frac{\mathrm{d} \log \xi}{\mathrm{d} \log A_{i}}-\sum_{j \neq i} \lambda_{j} \frac{\mathrm{d} \log \lambda_{j} / \lambda_{i}}{\mathrm{~d} \log A_{i}}-\sum_{j \neq i} \lambda_{j} \frac{\mathrm{d} \log \lambda_{i}}{\mathrm{~d} \log A_{i}}
$$

which we can rewrite as

$$
\lambda_{i} \frac{\mathrm{d} \log \lambda_{i}}{\mathrm{~d} \log A_{i}}=\xi \frac{\mathrm{d} \log \xi}{\mathrm{d} \log A_{i}}+\sum_{j \neq i} \lambda_{j}\left(1-\frac{1}{\rho_{j i}}\right)-\left(\xi-\lambda_{i}\right) \frac{\mathrm{d} \log \lambda_{i}}{\mathrm{~d} \log A_{i}},
$$

Rearrange this to get

$$
\xi \frac{\mathrm{d} \log \lambda_{i}}{\mathrm{~d} \log A_{i}}=\xi \frac{\mathrm{d} \log \xi}{\mathrm{d} \log A_{i}}+\sum_{j \neq i} \lambda_{j}\left(1-\frac{1}{\rho_{j i}}\right) .
$$

Finally, Theorem 1 implies that

$$
\frac{\mathrm{d}^{2} \log Y}{\mathrm{~d} \log \left(A_{i}\right)^{2}}=\lambda_{i} \frac{\mathrm{d} \log \lambda_{i}}{\mathrm{~d} \log A_{i}}
$$

Substitute (17) into the expression above to get the desired result. Lastly, if $Y$ is homogeneous, Euler's theorem implies that

$$
\sum_{i} \frac{\mathrm{d} Y}{\mathrm{~d} A_{i}} \frac{A_{i}}{Y}=\sum_{i} \lambda_{i}=\xi
$$

hence, $\mathrm{d} \log \xi / \mathrm{d} \log A_{i}=0$.

Proof of Proposition 4. We prove a slightly more general formulation with arbitrary variance covariance matrix and an arbitrary twice-differentiable utility function.

$$
\begin{aligned}
& E(u(Y(A))) \approx E\left(u(Y(\bar{A}))+u^{\prime}(Y(\bar{A})) \nabla Y(\bar{A})(A-\bar{A})+\frac{1}{2} u^{\prime \prime}(Y(\bar{A}))(A-\bar{A})^{\prime}\left(\nabla Y(\bar{A}) \circ \nabla Y(\bar{A})^{\prime}\right)(A-\bar{A})+\right. \\
&\left.\frac{1}{2} u^{\prime}(Y(\bar{A}))(A-\bar{A})^{\prime} \nabla^{2} Y(\bar{A})(A-\bar{A})\right) \\
&=u(Y(\bar{A}))+\frac{1}{2} u^{\prime \prime}(Y(\bar{A})) \operatorname{tr}\left(\left(\nabla Y(\bar{A}) \circ \nabla Y(\bar{A})^{\prime}\right) \Sigma\right)+\frac{1}{2} u^{\prime}(Y(\bar{A})) \operatorname{tr}\left(\nabla^{2} Y(\bar{A}) \Sigma\right) .
\end{aligned}
$$

Now apply Hulten's theorem to get

$$
=u(Y(\bar{A}))+\frac{1}{2} u^{\prime \prime}(Y(\bar{A})) \sum_{k, j}^{N} \lambda_{k} \lambda_{j} \sigma_{j k}+\frac{1}{2} u^{\prime}(Y(\bar{A})) \sum_{j, k}^{N} \frac{\mathrm{d}^{2} \Upsilon}{\mathrm{d} A_{k} \mathrm{~d} A_{j}} \sigma_{j k},
$$


with idiosyncratic shocks, this simplifies to

$$
=u(Y(\bar{A}))+\frac{1}{2} u^{\prime \prime}(Y(\bar{A})) \sum_{k}^{N} \lambda_{k}^{2} \sigma_{k}^{2}+\frac{1}{2} u^{\prime}(Y(\bar{A})) \sum_{k}^{N} \frac{\mathrm{d}^{2} Y}{\mathrm{~d} A_{k}^{2}} \sigma_{k}^{2} .
$$

The second summand is the Lucas term (which equals zero when $u$ is linear), and the third summand is our term. Rearrange this to get the desired result.

Proof of Proposition 3.

$$
\frac{\mathrm{d}^{2} \log Y}{\mathrm{~d} \log A_{j} \log A_{i}}=\frac{\mathrm{d} \lambda_{i}}{\mathrm{~d} \log A_{j}} .
$$

By definition

$$
\frac{\mathrm{d} \log \lambda_{i}}{\mathrm{~d} \log A_{j}}=\left(\frac{1}{\rho_{j i}}-1\right)+\frac{\mathrm{d} \log \lambda_{j}}{\mathrm{~d} \log A_{j}},
$$

which simplifies to

$$
\frac{\mathrm{d} \lambda_{i}}{\mathrm{~d} \log A_{j}}=\lambda_{i}\left(\frac{1}{\rho_{j i}}-1\right)+\frac{\lambda_{i}}{\lambda_{j}} \frac{\mathrm{d} \lambda_{j}}{\mathrm{~d} \log A_{j}} .
$$

Now apply theorem 2 to the second summand to obtain the desired result.

Proof of Proposition 5. The allocation for labor is

$$
\frac{l_{i g}}{\bar{l}_{i g}}=\left(\frac{\omega_{0 i} A_{i}^{\frac{\theta-1}{\theta}}}{\sum \omega_{0 j} A_{j}^{\frac{\theta-1}{\theta}}}\right)^{\frac{1}{1-\omega_{g} \frac{\theta-1}{\theta}}} .
$$

Substituting this into the utility function gives

$$
\frac{Y}{\bar{Y}}=\left(\sum_{i} \omega_{0 i}^{\frac{\theta}{\theta\left(1-\omega_{g}\right)+\omega_{g}}} A_{i}^{\frac{\theta-1}{\theta\left(1-\omega_{g}\right)+\omega_{g}}}\right)^{\frac{\theta\left(1-\omega_{g}\right)+\omega_{g}}{\theta-1}} .
$$

Then for this economy

$$
\rho_{j i}=\rho=\frac{\theta\left(1-\omega_{g}\right)+\omega_{g}}{\theta\left(1-\omega_{g}\right)+\omega_{g}+(1-\theta)},
$$

where $\omega_{g}=0$ corresponds to

$$
\rho=\theta,
$$


which is the same as no reallocation case. On the other hand, for $\omega_{g}=1$,

$$
\rho=\frac{1}{2-\theta}
$$

which is the same as the fully reallocative case. Note that this explodes when $\theta \geq 2$. For $\rho \in(0,1)$ we get something in between the perfectly reallocative and no reallocation special cases.

Proof of Proposition 6. Consumption is given by

$$
Y=A \bar{Y}\left(\bar{a}\left(\frac{l}{\bar{l}}\right)^{\frac{\theta-1}{\theta}}+(1-\bar{a})\left(\frac{X}{\bar{X}}\right)^{\frac{\theta-1}{\theta}}\right)^{\frac{\theta}{\theta-1}}-X .
$$

The first-order condition gives

$$
\frac{X}{\bar{X}}=(\bar{Y} A)^{\theta-1}(1-\bar{a})^{\theta} \bar{X}^{-\theta} Y
$$

Substituting this into the production function gives

$$
Y=\frac{A \bar{Y} \bar{a}^{\frac{\theta}{\theta-1}}}{\left(1-(1-\bar{a})^{\theta}(\bar{Y} A / \bar{X})^{\theta-1}\right)^{\frac{\theta}{\theta-1}}} .
$$

This means that

$$
Y=\frac{A \bar{Y} \bar{a}^{\frac{\theta}{\theta-1}}}{\left(1-(1-\bar{a})^{\theta}(\bar{Y} A / \bar{X})^{\theta-1}\right)^{\frac{1}{\theta-1}}} .
$$

Finally, note that

$$
\frac{\mathrm{d} \log Y}{\mathrm{~d} \log A}=\xi
$$

Proof of Proposition 7. This follows as a special case of Proposition 9.

Proof of Corollary 1. The proof is similar to that of Corollary 2.

Proof of Proposition 8. Denote the $i$ th standard basis vector by $e_{i}$. Then, by assumption, $\Omega e_{i}=\Omega e_{j}$ and $b^{\prime} e_{i}=b^{\prime} e_{j}$. Repeated multiplication implies that $\Omega^{n} e_{i}=\Omega^{n} e_{j}$. This then implies that $\Psi e_{i}=\Psi e_{j}$ so that in steady state, $\lambda_{i}=b^{\prime} \Psi e_{i}=b^{\prime} \Psi e_{j}=\lambda_{j}$. So the first-order impact of a shock is the same. Furthermore, substitution into (7) shows that the second-order impact of a shock is also the same. 
Proof of Proposition 9. Denote the $N \times F$ matrix corresponding to $\Omega_{i f}$ by $\alpha_{i f}$. By Shephard's lemma,

$$
\frac{\mathrm{d} \log p_{i}}{\mathrm{~d} \log A_{k}}=-\mathbf{1}(i=k)+\sum_{j} \Omega_{i j} \frac{\mathrm{d} \log p_{j}}{\mathrm{~d} \log A_{k}}+\sum_{f} \alpha_{i f} \frac{\mathrm{d} \log w_{f}}{\mathrm{~d} \log A_{k}} .
$$

Invert this system to get

$$
\frac{\mathrm{d} \log p_{i}}{\mathrm{~d} \log A_{k}}=-\Psi_{i k}+\sum_{f} \Psi_{i f} \frac{\mathrm{d} \log w_{f}}{\mathrm{~d} \log A_{k}}
$$

where $\Psi_{(f)}=(I-\Omega)^{-1} \alpha_{(f)}$. Note that $b^{\prime} \Psi_{f}=\Lambda_{f}$.

Denote the household's final demand expenditure share $\Omega_{0 i}$ by $b_{i}$. Then, for a factor $L$, we have

$$
\begin{aligned}
\frac{\mathrm{d} \Lambda_{f}}{\mathrm{~d} \log A_{k}}= & \sum_{i} b_{i}\left(1-\theta_{0}\right)\left[-\Psi_{i k}+\sum_{g} \Psi_{i g} \frac{\mathrm{d} \log w_{g}}{\mathrm{~d} \log A_{k}}\right] \Psi_{i f} \\
& +\sum_{j}\left(1-\theta_{j}\right) \lambda_{j} \sum_{i} \Omega_{j i}\left[-\Psi_{i k}+\sum_{g} \Psi_{i g} \frac{\mathrm{d} \log w_{g}}{\mathrm{~d} \log A_{k}}+\Psi_{j k}-\sum_{g} \Psi_{j g} \frac{\mathrm{d} \log w_{g}}{\mathrm{~d} \log A_{k}}\right] \Psi_{i f} \\
& +\left(\theta_{k}-1\right) \lambda_{k} \sum_{i} \Omega_{k i} \Psi_{i f} .
\end{aligned}
$$

Simplify this to

$$
\begin{aligned}
\mathrm{d} \Lambda_{f}= & \left(\theta_{0}-1\right)\left(\sum_{i} b_{i} \Psi_{i k} \Psi_{i f}-\sum_{i} b_{i} \Psi_{i f} \sum_{g} \Psi_{i g} \frac{\mathrm{d} \log w_{g}}{\mathrm{~d} \log A_{k}}\right), \\
& +\sum_{j}\left(\theta_{j}-1\right) \lambda_{j}\left[\sum_{i} \Omega_{j i} \Psi_{i k} \Psi_{i f}-\sum_{i} \Omega_{j i} \Psi_{j k} \Psi_{i f}\right] \\
& +\sum_{j}\left(1-\theta_{j}\right) \lambda_{j}\left[\sum_{i} \Omega_{j i} \sum_{g}\left(\Psi_{i g}-\Psi_{j g}\right) \frac{\mathrm{d} \log w_{g}}{\mathrm{~d} \log A_{k}} \Psi_{i f}\right] \\
& +\left(\theta_{k}-1\right) \lambda_{k} \sum_{i} \Omega_{k i} \Psi_{i f}, \\
= & \left(\theta_{0}-1\right)\left(\sum_{i} b_{i} \Psi_{i k} \Psi_{i f}-\sum_{i} b_{i} \Psi_{i f} \sum_{g} \Psi_{i g} \frac{\mathrm{d} \log w_{g}}{\mathrm{~d} \log A_{k}}\right), \\
& +\sum_{j}\left(\theta_{j}-1\right) \lambda_{j}\left[\sum_{i} \Omega_{j i} \Psi_{i k} \Psi_{i f}-\left(\sum_{i} \Omega_{j i} \Psi_{i f}\right)\left(\sum_{i} \Omega_{j i} \Psi_{i k}\right)\right] \\
& +\sum_{j}\left(1-\theta_{j}\right) \lambda_{j}\left[\sum_{i} \Omega_{j i} \sum_{g}\left(\Psi_{i g}-\Psi_{j g}\right) \frac{\mathrm{d} \log w_{g}}{\mathrm{~d} \log A_{k}} \Psi_{i f}\right],
\end{aligned}
$$




$$
\begin{aligned}
& =\left(\theta_{0}-1\right)\left(\sum_{i} b_{i} \Psi_{i k} \Psi_{i f}-\sum_{i} b_{i} \Psi_{i f} \sum_{g} \Psi_{i g} \frac{\mathrm{d} \log w_{g}}{\mathrm{~d} \log A_{k}}\right), \\
& +\sum_{j}\left(\theta_{j}-1\right) \lambda_{j} \operatorname{Cov}_{\Omega^{(j)}}\left(\Psi_{(k)}, \Psi_{(f)}\right) \\
& +\sum_{j}\left(1-\theta_{j}\right) \lambda_{j} \sum_{g}\left[\sum_{i} \Omega_{j i} \Psi_{i g} \Psi_{i f}-\left(\sum_{i} \Omega_{j i} \Psi_{i f}\right)\left(\sum_{i} \Omega_{j i} \Psi_{i g}\right)\right] \frac{\mathrm{d} \log w_{g}}{\mathrm{~d} \log A_{k}}, \\
& =\left(\theta_{0}-1\right)\left(\sum_{i} b_{i} \Psi_{i k} \Psi_{i f}-\sum_{i} b_{i} \Psi_{i f} \sum_{g} \Psi_{i g} \frac{\mathrm{d} \log w_{g}}{\mathrm{~d} \log A_{k}}\right), \\
& +\sum_{j}\left(\theta_{j}-1\right) \lambda_{j} \operatorname{Cov}_{\Omega^{(j)}}\left(\Psi_{(k)}, \Psi_{(f)}\right) \\
& +\sum_{j}\left(1-\theta_{j}\right) \lambda_{j} \sum_{g} \operatorname{Cov}_{\Omega^{(j)}}\left(\Psi_{(g)}, \Psi_{(f)}\right) \frac{\mathrm{d} \log w_{g}}{\mathrm{~d} \log A_{k}}, \\
& =\left(\theta_{0}-1\right)\left(\sum_{i} b_{i} \Psi_{i k} \Psi_{i f}-\sum_{i} b_{i} \Psi_{i f} \sum_{g} \Psi_{i g} \frac{\mathrm{d} \log w_{g}}{\mathrm{~d} \log A_{k}}\right), \\
& +\sum_{j}\left(\theta_{j}-1\right) \lambda_{j} \operatorname{Cov}_{\Omega^{(j)}}\left(\Psi_{(k)}-\sum_{g} \Psi_{(g)} \frac{\mathrm{d} \log w_{g}}{\mathrm{~d} \log A_{k}}, \Psi_{(f)}\right) \\
& =\left(\theta_{0}-1\right)\left(\sum_{i} b_{i} \Psi_{i f}\left(\Psi_{i k}-\sum_{g} \Psi_{i g} \frac{\mathrm{d} \log w_{g}}{\mathrm{~d} \log A_{k}}\right)\right) \text {, } \\
& +\sum_{j}\left(\theta_{j}-1\right) \lambda_{j} \operatorname{Cov}_{\Omega^{(j)}}\left(\Psi_{(k)}-\sum_{g} \Psi_{(g)} \frac{\mathrm{d} \log w_{g}}{\mathrm{~d} \log A_{k}}, \Psi_{(f)}\right) \\
& =\left(\theta_{0}-1\right) \operatorname{Cov}_{b}\left(\Psi_{(k)}-\sum_{g} \Psi_{(g)} \frac{\mathrm{d} \log w_{g}}{\mathrm{~d} \log A_{k}}, \Psi_{(f)}\right)+\left(\theta_{0}-1\right)\left(\lambda_{k}-\sum_{g} \Lambda_{g} \frac{\mathrm{d} \log w_{g}}{\mathrm{~d} \log A_{k}}\right) \lambda_{f}, \\
& +\sum_{j}\left(\theta_{j}-1\right) \lambda_{j} \operatorname{Cov}_{\Omega^{(j)}}\left(\Psi_{(k)}-\sum_{g} \Psi_{(g)} \frac{\mathrm{d} \log w_{g}}{\mathrm{~d} \log A_{k}}, \Psi_{(f)}\right) .
\end{aligned}
$$

Hence, for a productivity shock $\mathrm{d} \log A_{k}$, letting $\Lambda_{f}$ be demand for factor $f$, we have

$$
\begin{aligned}
\frac{\mathrm{d} \Lambda_{f}}{\mathrm{~d} \log A_{k}}= & \left(\theta_{0}-1\right) \operatorname{Cov}_{b}\left(\Psi_{(k)}-\sum_{g} \Psi_{(g)} \frac{\mathrm{d} \log w_{g}}{\mathrm{~d} \log A_{k}}, \Psi_{(f)}\right) \\
& +\sum_{j}\left(\theta_{j}-1\right) \lambda_{j} \operatorname{Cov}_{\Omega_{j}}\left(\Psi_{(k)}-\sum_{g} \Psi_{(g)} \frac{\mathrm{d} \log w_{g}}{\mathrm{~d} \log A_{k}}, \Psi_{(f)}\right) \\
& +\left(\theta_{0}-1\right)\left(\lambda_{k}-\sum_{g} \Lambda_{g} \frac{\mathrm{d} \log w_{g}}{\mathrm{~d} \log A_{k}}\right) \Lambda_{f}
\end{aligned}
$$


We have

$$
\frac{\mathrm{d} \log w_{f}}{\mathrm{~d} \log A_{k}}=\frac{\mathrm{d} \log Y}{\mathrm{~d} \log A_{k}}+\frac{1}{\Lambda_{f}} \frac{\mathrm{d} \Lambda_{f}}{\mathrm{~d} \log A_{k}}=\lambda_{k}+\frac{1}{\Lambda_{f}} \frac{\mathrm{d} \Lambda_{f}}{\mathrm{~d} \log A_{k}} .
$$

Substituting this expression for back into the formula, we get

$$
\begin{aligned}
\frac{\mathrm{d} \log \Lambda_{f}}{\mathrm{~d} \log A_{k}}= & \left(\theta_{0}-1\right) \frac{1}{\Lambda_{f}} \operatorname{Cov}_{b}\left(\Psi_{(k)}-\sum_{g} \Psi_{(g)} \mathrm{d} \log \Lambda_{g}, \Psi_{(f)}\right) \\
& +\sum_{j}\left(\theta_{j}-1\right) \frac{\lambda_{j}}{\Lambda_{f}} \operatorname{Cov}_{\Omega^{(j)}}\left(\Psi_{(k)}-\sum_{g} \Psi_{(g)} \frac{\mathrm{d} \log \Lambda_{g}}{\mathrm{~d} \log A_{k}}, \Psi_{(f)}\right) .
\end{aligned}
$$

The proof obtains by labelling final demand as producer 0 . The derivation of the expression for $\mathrm{d} \log \lambda_{i} / \mathrm{d} \log A_{k}$ is similar.

Proof of Corollary 2. Note that since $\theta_{i}=\theta$ for every $i$, market clearing for a good $i$ (neglecting the normalizing constants and setting the household's price index to be the numeraire), is

$$
p_{i}^{\theta} y_{i}=\omega_{0 i}^{\theta} Y+\sum_{j} p_{j}^{\theta} y_{j} \omega_{j i}^{\theta} .
$$

Hence, letting $p^{\theta} y$ denote the vector whose $i$ th element is $p_{i}^{\theta} y_{i}$ and $b_{i}=\omega_{0 i}$, we can write

$$
p^{\theta} y=b^{\prime}\left(I-\omega^{\circ \theta}\right)^{-1} Y
$$

where $\omega^{\circ \theta}$ is the matrix of $\omega_{i j}$ raised to $\theta$ elementwise. Let $\tilde{b}^{\prime}=b^{\prime}\left(I-\omega^{\circ \theta}\right)^{-1}$. This is reminiscent of the supplier centrality defined by Baqaee (2018).

Furthermore, market clearing for labor type $k$ is

$$
w_{k}^{\theta} \bar{l}_{k}=A_{k}^{-1} \sum_{i} p_{i}^{\theta} y_{i} \omega_{i k^{\prime}}^{\theta}
$$

where we use the fact that productivity shocks affect only the stock of factors. Rearrange this to get

$$
w_{k}^{\theta} \bar{l}_{k}=A_{k}^{-1} Y\left(\sum_{i} \tilde{b}_{i} \omega_{i k}^{\theta}\right)
$$

whence

$$
w_{k}=\left(\frac{Y}{A_{k} \bar{l}_{k}}\right)^{\frac{1}{\theta}}\left(\sum_{i} \tilde{b}_{i} \omega_{i k}^{\theta}\right)^{\frac{1}{\theta}} .
$$


To complete the proof note that

$$
Y=\sum_{k} w_{k} A_{k} \bar{l}_{k}=\left(\frac{Y}{A_{k} \bar{l}_{k}}\right)^{\frac{1}{\theta}}\left(\sum_{i} \tilde{b}_{i} \omega_{i k}^{\theta}\right)^{\frac{1}{\theta}} A_{k} \bar{l}_{k} .
$$

Rearrange this to get a closed form expression for output

$$
Y=\left(\sum_{k}\left(A_{k} \bar{l}_{k}\right)^{\frac{\theta-1}{\theta}}\left(\sum_{i} \tilde{b}_{i} \omega_{i k}^{\theta}\right)^{\frac{1}{\theta}}\right)^{\frac{\theta}{\theta-1}} .
$$

Since $Y$ can be written in closed-form as a CES aggregate of the underlying productivity shocks, Corollary 2 follows immediately.

Proof of Proposition 10. By Lemma (5.8) from (Theil, 1967, p.222) we know that

$$
\begin{aligned}
\log (Y(A+\Delta) / Y(A)) & \\
= & \frac{1}{2}[\nabla \log Y(A+\Delta)+\nabla \log Y(A)]^{\prime}[\log (A+\Delta)-\log (A)]+O\left(\Delta^{3}\right) .
\end{aligned}
$$

Hulten (1978) then implies that $\nabla \log Y(A)=\lambda(A)$ and $\nabla \log Y(A+\Delta)=\lambda(A+\Delta)$.

\section{B Generalization of Section 3.2 to Multiple Goods}

For the example is Section 3.2, the economy with extreme complementarity $\theta=0$ has $Y=$ $A / a$, where $1 / a$ is the sales to output ratio in steady-state. Therefore, in this example, although Hulten's approximation fails in log terms, Hulten's theorem is globally accurate in linear terms. In other words, our examples so far may suggest that extreme complementarities can only have outsized effects, in linear terms, if we restrict the movement of labor across industries.

However, this impression is false. To see this, consider a slightly more complex example where we generalize the example above by allowing multiple industries. Aggregate consumption is Cobb-Douglas across goods with equal weights $\left(b_{i}=1 / N\right)$. Each good is produced using labor and the good itself as an intermediate input. We assume full labor reallocation/constant returns to scale. We have

$$
Y=\prod_{i} c_{i}^{1 / N},
$$


and

$$
y_{i}=\bar{y}_{i} A_{i}\left(\omega_{i l}\left(\frac{l_{i}}{\bar{l}_{i}}\right)^{\frac{\theta_{i}-1}{\theta_{i}}}+\left(1 \omega_{i l}\right)\left(\frac{x_{i}}{\bar{x}_{i}}\right)^{\frac{\theta_{i}-1}{\theta_{i}}}\right)^{\frac{\theta_{i}}{\theta_{i}-1}}
$$

with

$$
y_{i}=c_{i}+x_{i}
$$

and perfect reallocation of labor. Then we have the following.

Proposition 11. Consider the model described above. Then

$$
1-\frac{1}{\rho_{j i}}=\left(\theta_{i}-1\right)\left(\frac{1}{\omega_{i l}}-1\right)
$$

and

$$
\frac{\mathrm{d} \log \xi}{\mathrm{d} \log A_{i}}=\frac{1}{N}\left(\theta_{i}-1\right)\left(\frac{1}{\omega_{i l}}-1\right) .
$$

In Figure 9 we plot output as a function of TFP shocks in linear terms. As promised, this economy features strong aggregate complementarities in the sense that a negative TFP shock can cause a drastic reduction in output even in linear terms, despite the fact that labor can be costlessly reallocated across sectors. This happens because, in equilibrium, a negative shock to industry $i$ does not result in more labor being allocated to production in industry $i$. This follows from the fact that consumption has a Cobb-Douglas form, and so the income and substitution effects from a shock to $i$ offset each other. Since no new labor is allocated to $i$, if $i$ faces a low structural elasticity of substitution $\theta_{i} \approx 0$, its output falls dramatically in response to a negative shock. This can then have a large effect on aggregate consumption. Of course, Cobb-Douglas consumption is simply a clean way to illustrate this intuition. If the structural elasticity of substitution in consumption where less than unity $\left(\theta_{0}<1\right)$, then these effects would be even further amplified.

Proof of Proposition 11. First, consider

$$
\max _{x_{i}} y_{i}-x_{i}
$$

which has the first-order condition

$$
x_{i}=y_{i}\left(1-\omega_{i l}\right)^{\theta_{i}}\left(\frac{A_{i} \bar{y}_{i}}{\bar{x}_{i}}\right)^{\theta_{i}-1}=y_{i}\left(1-\omega_{i l}\right) A_{i}^{\theta_{i}-1},
$$

where we use the fact that $\bar{X}_{i}=\bar{y}_{i}\left(1-\omega_{i l}\right)$. Substitute this into the production function for $y_{i}$ 


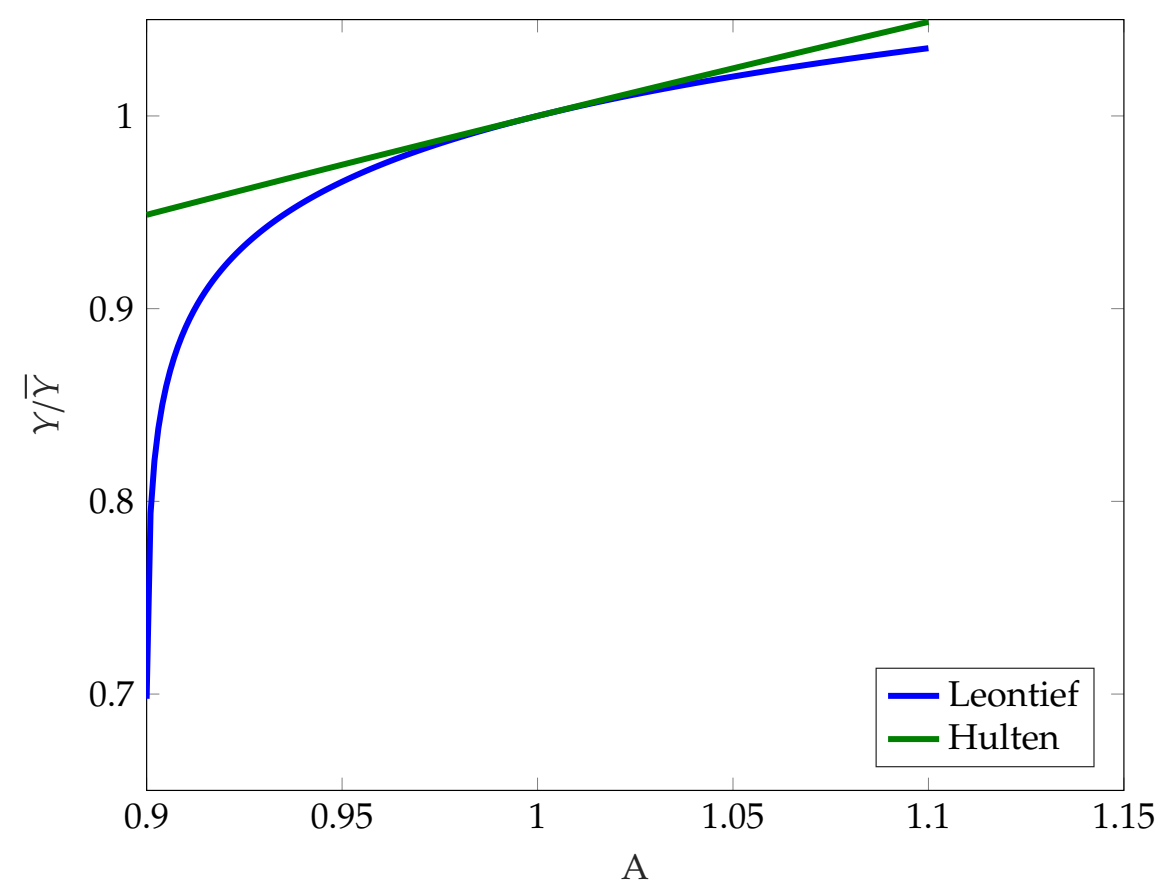

Figure 9: Aggregate output for the Leontief case $\theta_{i} \approx 0$ with two industries.

to get

$$
y_{i}=\frac{A_{i} \bar{y}_{i} \bar{a}^{\theta_{i} /\left(\theta_{i}-1\right)} l_{i} / \bar{l}_{i}}{\left(1-(1-\bar{a}) A_{i}^{\theta_{i}-1}\right)^{\frac{\theta_{i}}{\theta_{i}-1}}} .
$$

Substitute this into $c_{i}=y_{i}-x_{i}$ to get

$$
c_{i}=\frac{A_{i} \bar{y}_{i} \bar{a}^{\theta_{i} /\left(\theta_{i}-1\right)} l_{i} / \bar{l}_{i}}{\left(1-(1-\bar{a}) A_{i}^{\theta_{i}-1}\right)^{\frac{1}{\theta_{i}-1}}} .
$$

Substitute these into the utility function to get aggregate consumption when labor cannot be reallocated. To get aggregate consumption when labor is reallocated, maximize aggregate the non-reallocative solution with respect to $l_{i}$ :

$$
\frac{Y}{\bar{Y}}=\left(\sum_{i}^{N} \bar{b}_{i}^{\theta_{0}}\left(\frac{A_{i} \bar{y}_{i} \bar{a}_{i}^{\frac{\theta_{i}}{\theta_{i}-1}} / \bar{l}_{i}}{\left(1-\left(1-\omega_{i l}\right) A_{i}^{\theta_{i}-1}\right)^{\frac{1}{\theta_{i}-1}}}\right)^{\theta_{0}-1}\right)^{\frac{1}{\theta_{0}-1}} \bar{l} .
$$




\section{Adjustment Costs in the Quantitative Model}

In this section, we explain how to extend the quantitative model of Section 6.1 to allow for adjustment costs. For each composite intermediate input, we allow for the possibility that there are adjustment costs, indexed by $\kappa \geq 0$, in adjusting the quantity of the input compared to its steady-state value:

$$
\hat{X}_{i}=X_{i}\left(1-\frac{\kappa}{2}\left(\frac{X_{i}}{\bar{X}_{i}}-1\right)^{2}\right)
$$

where $X_{i}$ are units of good $i$ purchased and $\hat{X}_{i}$ are the units of good $i$ actually used. When $\kappa=0$, there are no adjustment costs.

Introducing adjustment costs increases the volatility of the Domar weights. For the model with adjustment costs, we choose the value of the adjustment cost parameter $\kappa$ so that, given the microecononomic elasticities of substitution, the model matches the volatility of the Domar weights at an annual frequency (when the model already overshoots without adjustment costs, we set them to zero). We then keep the same value of $\kappa$ when we move quadrennial frequency. By picking a suitable value for $\kappa$, even the model with fully mobile labor can match the volatility of the Domar weights. We report these results in Table 2. Interestingly, once we pick $\kappa$ to match the volatility of Domar weights at annual frequency, the model also roughly matches the volatility of the Domar weights at a quadrennial frequency. The results are consistent with what we found in Table 1 . In the final column of Table 2 we also report the value of resources destroyed by the adjustment cost directly

$$
\Delta=E\left(\frac{\sum_{i} p_{i}\left(X_{i}-\hat{X}_{i}\right)}{G D P}\right) .
$$

In all cases, the amount of resources destroyed directly by the adjustment costs are not large enough to mechanically drive the reductions in average aggregate output. For example, whereas at quadrennial frequency, the reduction in expected log aggregate output is around $1.5 \%-0.5 \% \approx 1.0 \%$, the value of the resources destroyed by the adjustment costs are less than $0.5 \%$.

\section{Additional Tables and Figures}




\begin{tabular}{lllrrrrr}
\hline$(\sigma, \theta, \epsilon, \kappa, t)$ & Mean & Std & Skewness & Ex-Kurtosis & $\sigma_{\lambda}$ & \multicolumn{1}{c}{$\Delta$} \\
\hline \hline No reallocation & & & & & & & \\
$(0.9,0.5,0.001,0,1)$ & -0.0034 & 0.012 & -0.18 & 0.1 & 0.115 & 0 \\
$(0.9,0.5,0.001,0,4)$ & -0.0187 & 0.030 & -1.11 & 3.6 & 0.267 & 0 \\
$(0.9,0.6,0.2,2,1)$ & -0.0033 & 0.011 & -0.27 & 0.21 & 0.124 & 0.0007 \\
$(0.9,0.6,0.2,2,4)$ & -0.0152 & 0.028 & -0.63 & 1.57 & 0.286 & 0.0046 \\
\hline Full reallocation & & & & & & \\
$(0.9,0.5,0.001,3,1)$ & -0.0031 & 0.012 & -0.25 & 0.26 & 0.124 & 0.0006 \\
$(0.9,0.5,0.001,3,4)$ & -0.0166 & 0.030 & -0.98 & 2.47 & 0.279 & 0.0046 \\
$(0.9,0.6,0.2,4,1)$ & -0.0026 & 0.011 & -0.23 & 0.23 & 0.129 & 0.0004 \\
$(0.9,0.6,0.2,4,4)$ & -0.0140 & 0.029 & -0.75 & 1.05 & 0.291 & 0.0028 \\
\hline
\end{tabular}

Table 2: Simulated and estimated moments for the model with adjustment costs. The simulated moments are calculated from 10,000 draws. The parameter $t$ measures the length of the time interval for the shocks: annual and quadrennial. Finally, the column $\Delta$ is the share of lost resources. 
Mean Std Skewness Ex-Kurtosis

\begin{tabular}{lllll}
\hline \hline & & & & \\
No reallocation, Annual & -0.0031 & 0.011 & -0.16 & 0.1 \\
No reallocation, Quadrennial & -0.0173 & 0.027 & -0.60 & 1.0 \\
Full Reallocation, Annual & -0.0021 & 0.011 & -0.09 & 0.0 \\
Full Reallocation, Quadrennial & -0.0110 & 0.026 & -0.25 & 0.1 \\
\hline
\end{tabular}

Table 3: Moments of log output estimated from 50,000 draws using the second order Taylor approximation with the benchmark elasticities $(\sigma, \theta, \varepsilon)=(0.9,0.5,0.001)$. This is the version of the model with no adjustment $\cos t s \kappa=0$.

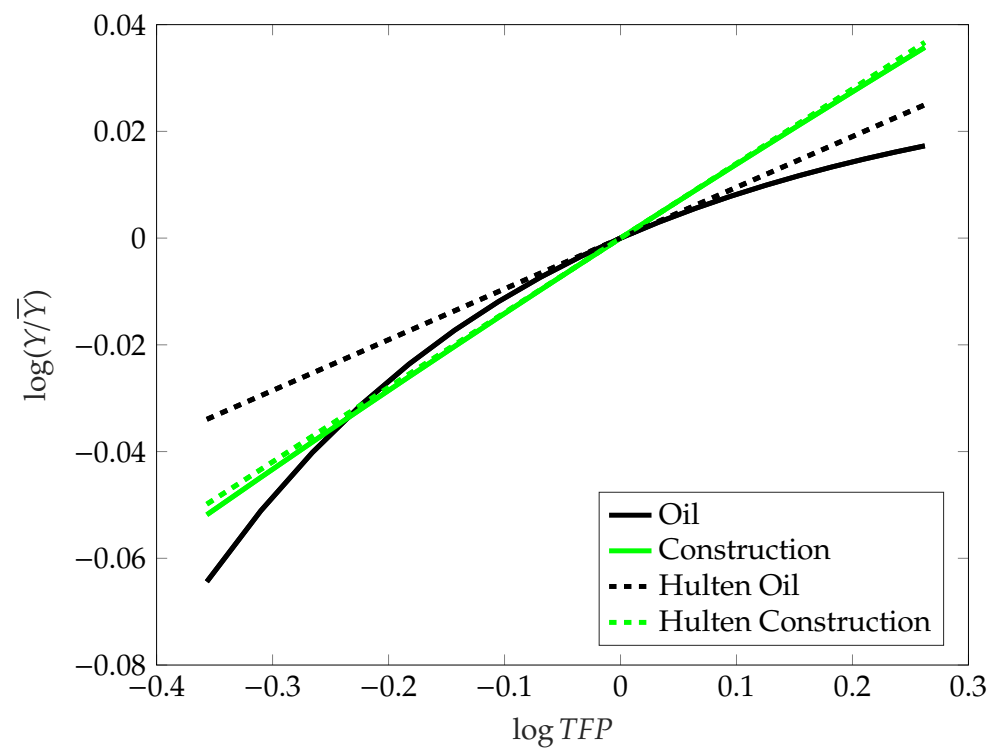

Figure 10: The effect of TFP shocks to the "oil and gas" industry and the construction industry. Construction has a bigger sales share, but "oil and gas" is more important for large negative shocks. This graph shows that the ranking of which industry is more important is not monotonic in the size of the shock. 


\begin{tabular}{|c|c|c|c|c|c|}
\hline$(\sigma, \theta, \epsilon)$ & Mean & Std & Skew & Ex-Kurtosis & $\sigma_{\lambda}$ \\
\hline 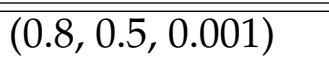 & -0.0023 & 0.011 & -0.06 & 0.0 & 0.074 \\
\hline$(0.9,0.5,0.001)$ & -0.0022 & 0.011 & -0.08 & 0.0 & 0.069 \\
\hline$(0.99,0.5,0.001)$ & -0.0021 & 0.011 & -0.07 & 0.0 & 0.065 \\
\hline$(0.8,0.5,0.2)$ & -0.0020 & 0.011 & -0.07 & 0.0 & 0.066 \\
\hline$(0.9,0.5,0.2)$ & -0.0020 & 0.011 & -0.08 & 0.0 & 0.062 \\
\hline$(0.99,0.5,0.2)$ & -0.0019 & 0.011 & -0.06 & 0.0 & 0.058 \\
\hline$(0.8,0.5,0.99)$ & -0.0014 & 0.011 & -0.02 & 0.0 & 0.044 \\
\hline$(0.9,0.5,0.99)$ & -0.0013 & 0.011 & -0.03 & 0.0 & 0.040 \\
\hline$(0.99,0.5,0.99)$ & -0.0013 & 0.011 & -0.02 & 0.0 & 0.036 \\
\hline$(0.8,0.4,0.001)$ & -0.0023 & 0.011 & -0.08 & 0.0 & 0.079 \\
\hline$(0.9,0.4,0.001)$ & -0.0022 & 0.011 & -0.06 & 0.0 & 0.075 \\
\hline$(0.99,0.4,0.001)$ & -0.0022 & 0.011 & -0.07 & 0.0 & 0.071 \\
\hline$(0.8,0.4,0.2)$ & -0.0021 & 0.011 & -0.06 & 0.0 & 0.073 \\
\hline$(0.9,0.4,0.2)$ & -0.0021 & 0.011 & -0.08 & 0.0 & 0.068 \\
\hline$(0.99,0.4,0.2)$ & -0.0020 & 0.011 & -0.07 & 0.0 & 0.064 \\
\hline$(0.8,0.4,0.99)$ & -0.0013 & 0.011 & -0.04 & 0.0 & 0.052 \\
\hline$(0.9,0.4,0.99)$ & -0.0014 & 0.011 & -0.04 & 0.0 & 0.047 \\
\hline$(0.99,0.4,0.99)$ & -0.0013 & 0.011 & -0.01 & 0.0 & 0.044 \\
\hline$(0.8,0.6,0.001)$ & -0.0022 & 0.011 & -0.06 & 0.0 & 0.068 \\
\hline$(0.9,0.6,0.001)$ & -0.0021 & 0.011 & -0.08 & 0.0 & 0.063 \\
\hline$(0.99,0.6,0.001)$ & -0.0020 & 0.011 & -0.07 & 0.0 & 0.059 \\
\hline$(0.8,0.6,0.2)$ & -0.0021 & 0.011 & -0.05 & 0.0 & 0.061 \\
\hline$(0.9,0.6,0.2)$ & -0.0020 & 0.011 & -0.05 & 0.0 & 0.056 \\
\hline$(0.99,0.6,0.2)$ & -0.0020 & 0.011 & -0.04 & 0.0 & 0.052 \\
\hline$(0.8,0.6,0.99)$ & -0.0014 & 0.011 & -0.02 & 0.0 & 0.037 \\
\hline$(0.9,0.6,0.99)$ & -0.0013 & 0.011 & -0.02 & 0.0 & 0.033 \\
\hline$(0.99,0.6,0.99)$ & -0.0013 & 0.011 & -0.01 & 0.0 & 0.029 \\
\hline$(0.8,0.99,0.001)$ & -0.0022 & 0.011 & -0.09 & 0.0 & 0.052 \\
\hline$(0.9,0.99,0.001)$ & -0.0020 & 0.011 & -0.05 & 0.0 & 0.047 \\
\hline$(0.99,0.99,0.001)$ & -0.0021 & 0.011 & -0.06 & 0.0 & 0.044 \\
\hline$(0.8,0.99,0.2)$ & -0.0021 & 0.011 & -0.04 & 0.0 & 0.043 \\
\hline$(0.9,0.99,0.2)$ & -0.0019 & 0.011 & -0.05 & 0.0 & 0.039 \\
\hline$(0.99,0.99,0.2)$ & -0.0018 & 0.011 & -0.04 & 0.0 & 0.035 \\
\hline$(0.8,0.99,0.99)$ & -0.0013 & 0.011 & -0.03 & 0.0 & 0.011 \\
\hline$(0.9,0.99,0.99)$ & -0.0013 & 0.011 & -0.02 & 0.0 & 0.006 \\
\hline$(0.99,0.99,0.99)$ & -0.0013 & 0.011 & 0.01 & 0.0 & 0.001 \\
\hline
\end{tabular}

Table 4: Annual Shocks, Model with full reallocation and no adjustment costs. 


\begin{tabular}{|c|c|c|c|c|c|}
\hline$(\sigma, \theta, \epsilon)$ & Mean & Std & Skew & Ex-Kurtosis & $\sigma_{\lambda}$ \\
\hline 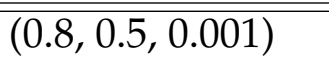 & -0.0112 & 0.026 & -0.31 & 0.3 & 0.178 \\
\hline$(0.9,0.5,0.001)$ & -0.0113 & 0.026 & -0.28 & 0.4 & 0.176 \\
\hline$(0.99,0.5,0.001)$ & -0.0107 & 0.026 & -0.27 & 0.3 & 0.163 \\
\hline$(0.8,0.5,0.2)$ & -0.0102 & 0.026 & -0.25 & 0.2 & 0.162 \\
\hline$(0.9,0.5,0.2)$ & -0.0101 & 0.026 & -0.23 & 0.1 & 0.152 \\
\hline$(0.99,0.5,0.2)$ & -0.0098 & 0.026 & -0.22 & 0.2 & 0.144 \\
\hline$(0.8,0.5,0.99)$ & -0.0070 & 0.025 & -0.09 & 0.0 & 0.113 \\
\hline$(0.9,0.5,0.99)$ & -0.0066 & 0.025 & -0.09 & 0.1 & 0.103 \\
\hline$(0.99,0.5,0.99)$ & -0.0064 & 0.025 & -0.09 & 0.0 & 0.095 \\
\hline$(0.8,0.4,0.001)$ & -0.0116 & 0.026 & -0.32 & 0.3 & 0.228 \\
\hline$(0.9,0.4,0.001)$ & -0.0110 & 0.026 & -0.32 & 0.3 & 0.228 \\
\hline$(0.99,0.4,0.001)$ & -0.0107 & 0.026 & -0.27 & 0.3 & 0.212 \\
\hline$(0.8,0.4,0.2)$ & -0.0106 & 0.026 & -0.27 & 0.3 & 0.201 \\
\hline$(0.9,0.4,0.2)$ & -0.0104 & 0.026 & -0.24 & 0.2 & 0.195 \\
\hline$(0.99,0.4,0.2)$ & -0.0097 & 0.026 & -0.25 & 0.2 & 0.173 \\
\hline$(0.8,0.4,0.99)$ & -0.0072 & 0.025 & -0.09 & 0.0 & 0.134 \\
\hline$(0.9,0.4,0.99)$ & -0.0070 & 0.025 & -0.09 & 0.0 & 0.125 \\
\hline$(0.99,0.4,0.99)$ & -0.0067 & 0.025 & -0.08 & 0.0 & 0.117 \\
\hline$(0.8,0.6,0.001)$ & -0.0112 & 0.026 & -0.26 & 0.2 & 0.159 \\
\hline$(0.9,0.6,0.001)$ & -0.0108 & 0.026 & -0.27 & 0.3 & 0.149 \\
\hline$(0.99,0.6,0.001)$ & -0.0105 & 0.026 & -0.26 & 0.2 & 0.140 \\
\hline$(0.8,0.6,0.2)$ & -0.0102 & 0.026 & -0.23 & 0.2 & 0.143 \\
\hline$(0.9,0.6,0.2)$ & -0.0100 & 0.026 & -0.23 & 0.2 & 0.133 \\
\hline$(0.99,0.6,0.2)$ & -0.0096 & 0.026 & -0.20 & 0.1 & 0.123 \\
\hline$(0.8,0.6,0.99)$ & -0.0071 & 0.025 & -0.07 & 0.0 & 0.093 \\
\hline$(0.9,0.6,0.99)$ & -0.0066 & 0.025 & -0.06 & 0.0 & 0.083 \\
\hline$(0.99,0.6,0.99)$ & -0.0064 & 0.025 & -0.06 & 0.0 & 0.075 \\
\hline$(0.8,0.99,0.001)$ & -0.0106 & 0.026 & -0.20 & 0.1 & 0.112 \\
\hline$(0.9,0.99,0.001)$ & -0.0104 & 0.026 & -0.19 & 0.1 & 0.103 \\
\hline$(0.99,0.99,0.001)$ & -0.0101 & 0.026 & -0.19 & 0.1 & 0.096 \\
\hline$(0.8,0.99,0.2)$ & -0.0100 & 0.025 & -0.15 & 0.1 & 0.093 \\
\hline$(0.9,0.99,0.2)$ & -0.0095 & 0.026 & -0.14 & 0.1 & 0.085 \\
\hline$(0.99,0.99,0.2)$ & -0.0091 & 0.026 & -0.13 & 0.1 & 0.078 \\
\hline$(0.8,0.99,0.99)$ & -0.0064 & 0.025 & -0.02 & 0.0 & 0.024 \\
\hline$(0.9,0.99,0.99)$ & -0.0062 & 0.025 & -0.01 & 0.0 & 0.013 \\
\hline$(0.99,0.99,0.99)$ & -0.0058 & 0.025 & 0.01 & 0.0 & 0.003 \\
\hline
\end{tabular}

Table 5: Quadrennial Shocks, model with full reallocation and no adjustment costs. 


\begin{tabular}{|c|c|c|c|c|c|}
\hline$(\sigma, \theta, \epsilon)$ & Mean & Std & Skew & Ex-Kurtosis & $\sigma_{\lambda}$ \\
\hline 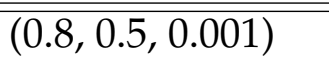 & -0.0036 & 0.011 & -0.23 & 0.2 & 0.128 \\
\hline$(0.9,0.5,0.001)$ & -0.0034 & 0.012 & -0.18 & 0.1 & 0.115 \\
\hline$(0.99,0.5,0.001)$ & -0.0032 & 0.011 & -0.20 & 0.1 & 0.104 \\
\hline$(0.8,0.5,0.2)$ & -0.0026 & 0.011 & -0.13 & 0.1 & 0.079 \\
\hline$(0.9,0.5,0.2)$ & -0.0026 & 0.011 & -0.11 & 0.0 & 0.070 \\
\hline$(0.99,0.5,0.2)$ & -0.0025 & 0.011 & -0.13 & 0.0 & 0.063 \\
\hline$(0.8,0.5,0.99)$ & -0.0014 & 0.011 & -0.01 & 0.0 & 0.018 \\
\hline$(0.9,0.5,0.99)$ & -0.0014 & 0.011 & -0.01 & 0.0 & 0.014 \\
\hline$(0.99,0.5,0.99)$ & -0.0012 & 0.011 & -0.01 & 0.0 & 0.011 \\
\hline$(0.8,0.4,0.001)$ & -0.0039 & 0.012 & -0.23 & 0.2 & 0.137 \\
\hline$(0.9,0.4,0.001)$ & -0.0035 & 0.011 & -0.21 & 0.2 & 0.123 \\
\hline$(0.8,0.4,0.2)$ & -0.0028 & 0.011 & -0.14 & 0.1 & 0.082 \\
\hline$(0.9,0.4,0.2)$ & -0.0026 & 0.011 & -0.12 & 0.1 & 0.073 \\
\hline$(0.99,0.4,0.2)$ & -0.0030 & 0.011 & 0.15 & 5.9 & 0.065 \\
\hline$(0.8,0.4,0.99)$ & -0.0015 & 0.011 & -0.05 & 0.0 & 0.020 \\
\hline$(0.9,0.4,0.99)$ & -0.0013 & 0.011 & -0.05 & 0.0 & 0.016 \\
\hline$(0.99,0.4,0.99)$ & -0.0014 & 0.011 & -0.04 & 0.0 & 0.014 \\
\hline$(0.8,0.6,0.001)$ & -0.0034 & 0.011 & -0.20 & 0.1 & 0.122 \\
\hline$(0.9,0.6,0.001)$ & -0.0032 & 0.011 & -0.20 & 0.1 & 0.109 \\
\hline$(0.99,0.6,0.001)$ & -0.0030 & 0.011 & -0.14 & 0.1 & 0.098 \\
\hline$(0.8,0.6,0.2)$ & -0.0026 & 0.011 & -0.12 & 0.0 & 0.077 \\
\hline$(0.9,0.6,0.2)$ & -0.0024 & 0.011 & -0.11 & 0.1 & 0.068 \\
\hline$(0.99,0.6,0.2)$ & -0.0023 & 0.011 & -0.10 & 0.0 & 0.061 \\
\hline$(0.8,0.6,0.99)$ & -0.0015 & 0.011 & -0.05 & 0.0 & 0.016 \\
\hline$(0.9,0.6,0.99)$ & -0.0013 & 0.011 & 0.00 & 0.0 & 0.011 \\
\hline$(0.99,0.6,0.99)$ & -0.0013 & 0.011 & -0.02 & 0.0 & 0.009 \\
\hline$(0.8,0.99,0.001)$ & -0.0030 & 0.011 & -0.15 & 0.1 & 0.107 \\
\hline$(0.9,0.99,0.001)$ & -0.0028 & 0.011 & -0.13 & 0.1 & 0.095 \\
\hline$(0.99,0.99,0.001)$ & -0.0027 & 0.011 & -0.11 & 0.1 & 0.086 \\
\hline$(0.8,0.99,0.2)$ & -0.0026 & 0.011 & -0.11 & 0.0 & 0.072 \\
\hline$(0.9,0.99,0.2)$ & -0.0024 & 0.011 & -0.09 & 0.0 & 0.063 \\
\hline$(0.99,0.99,0.2)$ & -0.0022 & 0.011 & -0.07 & 0.0 & 0.056 \\
\hline$(0.8,0.99,0.99)$ & -0.0014 & 0.011 & -0.01 & 0.0 & 0.010 \\
\hline$(0.9,0.99,0.99)$ & -0.0013 & 0.011 & -0.01 & 0.0 & 0.005 \\
\hline$(0.99,0.99,0.99)$ & -0.0011 & 0.011 & 0.00 & 0.0 & 0.001 \\
\hline
\end{tabular}

Table 6: Annual Shocks, model with no labor reallocation and no adjustment costs. 


\begin{tabular}{|c|c|c|c|c|c|}
\hline$(\sigma, \theta, \epsilon)$ & Mean & Std & Skew & Ex-Kurtosis & $\sigma_{\lambda}$ \\
\hline$(0.8,0.5,0.001)$ & -0.0202 & 0.031 & -1.26 & 4.5 & 0.297 \\
\hline$(0.9,0.5,0.001)$ & -0.0187 & 0.030 & -1.11 & 3.6 & 0.267 \\
\hline$(0.8,0.5,0.2)$ & -0.0139 & 0.028 & -0.58 & 1.1 & 0.180 \\
\hline$(0.9,0.5,0.2)$ & -0.0133 & 0.027 & -0.52 & 0.9 & 0.160 \\
\hline$(0.99,0.5,0.2)$ & -0.0176 & 0.024 & -0.66 & 1.3 & 0.138 \\
\hline$(0.8,0.5,0.99)$ & -0.0073 & 0.025 & -0.09 & 0.0 & 0.041 \\
\hline$(0.9,0.5,0.99)$ & -0.0068 & 0.025 & -0.07 & 0.0 & 0.033 \\
\hline$(0.99,0.5,0.99)$ & -0.0068 & 0.025 & -0.09 & 0.0 & 0.027 \\
\hline$(0.8,0.4,0.001)$ & -0.0217 & 0.032 & -1.40 & 5.3 & 0.320 \\
\hline$(0.9,0.4,0.001)$ & -0.0201 & 0.031 & -1.30 & 4.8 & 0.287 \\
\hline$(0.8,0.4,0.2)$ & -0.0146 & 0.028 & -0.67 & 1.4 & 0.187 \\
\hline$(0.9,0.4,0.2)$ & -0.0137 & 0.028 & -0.59 & 1.1 & 0.167 \\
\hline$(0.8,0.4,0.99)$ & -0.0075 & 0.025 & -0.13 & 0.0 & 0.048 \\
\hline$(0.9,0.4,0.99)$ & -0.0069 & 0.025 & -0.10 & 0.0 & 0.039 \\
\hline$(0.99,0.4,0.99)$ & -0.0069 & 0.025 & -0.09 & 0.0 & 0.034 \\
\hline$(0.8,0.6,0.001)$ & -0.0188 & 0.030 & -0.99 & 2.5 & 0.281 \\
\hline$(0.9,0.6,0.001)$ & -0.0176 & 0.029 & -0.90 & 2.2 & 0.253 \\
\hline$(0.99,0.6,0.001)$ & -0.0163 & 0.028 & -0.65 & 1.0 & 0.229 \\
\hline$(0.8,0.6,0.2)$ & -0.0136 & 0.027 & -0.49 & 0.7 & 0.175 \\
\hline$(0.9,0.6,0.2)$ & -0.0129 & 0.027 & -0.44 & 0.7 & 0.154 \\
\hline$(0.99,0.6,0.2)$ & -0.0128 & 0.026 & -0.50 & 0.6 & 0.138 \\
\hline$(0.8,0.6,0.99)$ & -0.0070 & 0.025 & -0.08 & 0.0 & 0.036 \\
\hline$(0.9,0.6,0.99)$ & -0.0066 & 0.025 & -0.08 & 0.0 & 0.027 \\
\hline$(0.99,0.6,0.99)$ & -0.0067 & 0.025 & -0.09 & 0.1 & 0.021 \\
\hline$(0.8,0.99,0.001)$ & -0.0163 & 0.028 & -0.64 & 1.1 & 0.246 \\
\hline$(0.9,0.99,0.001)$ & -0.0153 & 0.028 & -0.58 & 0.9 & 0.221 \\
\hline$(0.99,0.99,0.001)$ & -0.0145 & 0.027 & -0.53 & 0.8 & 0.200 \\
\hline$(0.8,0.99,0.2)$ & -0.0128 & 0.026 & -0.40 & 0.4 & 0.162 \\
\hline$(0.9,0.99,0.2)$ & -0.0120 & 0.026 & -0.35 & 0.3 & 0.143 \\
\hline$(0.99,0.99,0.2)$ & -0.0114 & 0.026 & -0.29 & 0.3 & 0.127 \\
\hline$(0.8,0.99,0.99)$ & -0.0066 & 0.025 & -0.01 & 0.0 & 0.021 \\
\hline$(0.9,0.99,0.99)$ & -0.0061 & 0.025 & -0.03 & 0.0 & 0.011 \\
\hline$(0.99,0.99,0.99)$ & -0.0057 & 0.025 & 0.00 & 0.0 & 0.002 \\
\hline
\end{tabular}

Table 7: Quadrennial Shocks, model with no reallocation and no adjustment costs. 


\section{E Macro Moment Approximations}

The notes in this section were prepared with the assistance of a research assistant Chang He. Let output be $Y(A)$, where $A$ is the $N \times 1$ vector of productivity parameters. Suppose that $A$ is distributed according to a multivariate normal distribution, and that the elements of $A$ are independent. Let $Y^{*}(A)$ be the second-order Taylor approximation of $Y$ around the mean vector of $A$.

\section{Second-order Taylor Approximation}

Let $\mu_{A}$ denote the mean vector of $A$. The second-order Taylor expansion of $Y(A)$ is:

$$
\Upsilon^{*}(\boldsymbol{A})=\Upsilon\left(\mu_{A}\right)+\sum_{i=1}^{N} \frac{\partial \Upsilon\left(\mu_{A}\right)}{\partial A_{i}}\left(A_{i}-\mu_{A_{i}}\right)+\frac{1}{2} \sum_{i=1}^{N} \sum_{j=1}^{N} \frac{\partial^{2} \Upsilon\left(\mu_{A}\right)}{\partial A_{i} \partial A_{j}}\left(A_{i}-\mu_{A_{i}}\right)\left(A_{j}-\mu_{A_{j}}\right) .
$$

We introduce the following abbreviations:

$$
\begin{gathered}
Y_{i}=\frac{\partial Y\left(\mu_{A}\right)}{\partial A_{i}}, \quad Y_{i j}=\frac{\partial^{2} Y\left(\mu_{A}\right)}{\partial A_{i} \partial A_{j}}, \\
\mu_{A_{i}}=\int_{-\infty}^{\infty} A_{i} f_{A}\left(A_{i}\right) d A_{i} \quad \mu_{A_{i, k}}=\int_{-\infty}^{\infty}\left(A_{i}-\mu_{A_{i}}\right)^{k} f_{A}\left(A_{i}\right) d A_{i}, \\
\mu_{A_{i}, A_{j}} \int_{-\infty}^{\infty} \int_{-\infty}^{\infty}\left(A_{i}-\mu_{A_{i}}\right)\left(A_{j}-\mu_{A_{j}}\right) f_{A}\left(A_{i}, A_{j}\right) d A_{i} d A_{j},
\end{gathered}
$$

where $f_{A}$ is the density function of $A$.

\section{Mean Value Approximation}

Let $\mu_{Y^{*}}$ be the mean value approximation of $Y(A)$. We have:

$$
\begin{aligned}
\boldsymbol{\mu}_{\Upsilon^{*}} & =E\left[\Upsilon^{*}(\boldsymbol{A})\right]=\int_{-\infty}^{\infty} \Upsilon^{*}(\boldsymbol{A}) f_{\boldsymbol{A}}(\boldsymbol{A}) d \boldsymbol{A}, \\
& =\int_{-\infty}^{\infty}\left[\Upsilon\left(\mu_{\boldsymbol{A}}\right)+\sum_{i=1}^{N} Y_{i}\left(A_{i}-\mu_{A_{i}}\right)+\frac{1}{2} \sum_{i=1}^{N} \sum_{j=1}^{N} Y_{i j}\left(A_{i}-\mu_{A_{i}}\right)\left(A_{j}-\mu_{A_{j}}\right)\right] f_{A}(\boldsymbol{A}) d \boldsymbol{A}, \\
& =\Upsilon\left(\mu_{A}\right)+\frac{1}{2} \sum_{i=1}^{N} \sum_{j=1}^{N} Y_{i j} \mu_{A_{i}, A_{j}} .
\end{aligned}
$$


Expanding the quadratic and since elements of $A$ are independent, we get

$$
\boldsymbol{\mu}_{\Upsilon^{*}}=\Upsilon\left(\mu_{A}\right)+\frac{1}{2} \sum_{i=1}^{N} Y_{i i} \mu_{A_{i}, 2}
$$

\section{Variance Approximation}

Let $\sigma_{Y^{*}}^{2}$ be the variance approximation of $Y(A)$.

$$
\begin{aligned}
\boldsymbol{\sigma}_{Y^{*}}^{2} & =E\left(\left[Y^{*}(\boldsymbol{A})-Y\left(\mu_{A}\right)\right]^{2}\right)=E\left(\Upsilon^{* 2}(\boldsymbol{A})\right)-\Upsilon^{2}\left(\mu_{A}\right), \\
& =\int_{-\infty}^{\infty}\left[Y\left(\mu_{A}\right)+\sum_{i=1}^{N} Y_{i}\left(A_{i}-\mu_{A_{i}}\right)+\frac{1}{2} \sum_{i=1}^{N} \sum_{j=1}^{N} Y_{i j}\left(A_{i}-\mu_{A_{i}}\right)\left(A_{j}-\mu_{A_{j}}\right)\right]^{2} f_{A}(\boldsymbol{A}) d \boldsymbol{A}-\boldsymbol{\mu}_{\Upsilon^{*}}^{2} .
\end{aligned}
$$

Since elements of $A$ are independent, we get

$$
\begin{aligned}
\sigma_{Y^{*}}^{2}=\sum_{i=1}^{N} Y_{i}^{2} \mu_{A_{i}, 2}+Y^{2}\left(\mu_{A}\right) & -\mu_{Y^{*}}^{2}+Y\left(\mu_{A}\right) \sum_{i=1}^{N} Y_{i i} \mu_{A_{i}, 2}+\sum_{i=1}^{N} Y_{i} Y_{i i} \mu_{A_{i}, 3} \\
& +\frac{1}{4} \sum_{i=1}^{N} Y_{i i}^{2} \mu_{A_{i}, 4}+\frac{1}{2} \sum_{i=1}^{N} \sum_{j=i+1}^{N} Y_{i i} Y_{j j} \mu_{A_{i}, 2} \mu_{A_{j}, 2}+\sum_{i=1}^{N} \sum_{j=i+1}^{N} Y_{i j}^{2} \mu_{A_{i,}, 2} \mu_{A_{j}, 2} .
\end{aligned}
$$

\section{Skewness Approximation}

Let $\boldsymbol{v}_{\Upsilon^{*}}$ be the skewness approximation of $Y(A)$. By definition, $\boldsymbol{v}_{\Upsilon^{*}}=\mu_{\Upsilon^{*}, 3} / \sigma_{Y^{*}}^{3}$.

Use the definition of skewness, and that $\int_{-\infty}^{\infty} Y^{* 2}(A) f_{A}(A) d A=\sigma_{\gamma^{*}}^{2}+\mu_{\gamma^{*}}^{2}$, we have

$$
\begin{aligned}
\boldsymbol{\mu}_{\Upsilon^{*}, 3} & =E\left(\left[Y^{*}(\boldsymbol{A})-Y\left(\mu_{A}\right)\right]^{3}\right)=\int_{-\infty}^{\infty}\left[Y^{*}(\boldsymbol{A})-Y\left(\mu_{A}\right)\right]^{3} f_{\boldsymbol{A}}(\boldsymbol{A}) d \boldsymbol{A}, \\
& =\int_{-\infty}^{\infty} \Upsilon^{* 3}(\boldsymbol{A}) f_{A}(\boldsymbol{A}) d \boldsymbol{A}-3 \boldsymbol{\mu}_{\Upsilon^{*}} \boldsymbol{\sigma}_{\Upsilon^{*}}^{2}-\boldsymbol{\mu}_{\Upsilon^{*}}^{3} \\
& =\int_{-\infty}^{\infty}\left[Y\left(\mu_{A}\right)+\sum_{i=1}^{N} Y_{i}\left(A_{i}-\mu_{A_{i}}\right)+\frac{1}{2} \sum_{i=1}^{N} \sum_{j=1}^{N} Y_{i j}\left(A_{i}-\mu_{A_{i}}\right)\left(A_{j}-\mu_{A_{j}}\right)\right] f_{\boldsymbol{A}}(\boldsymbol{A}) d \boldsymbol{A}-3 \boldsymbol{\mu}_{\Upsilon^{*}} \boldsymbol{\sigma}_{Y^{*}}^{2}-\boldsymbol{\mu}_{\Upsilon^{*}}^{3}
\end{aligned}
$$

Simplifying the equation above and use the fact that the elements of $A$ are independent, we have:

$$
\mu_{\Upsilon^{*}, 3}=\sum_{i=1}^{N} Y_{i}^{3} \mu_{A_{i}, 3}+Y^{3}\left(\mu_{A}\right)+\frac{3}{2} Y^{2}\left(\mu_{A}\right) \sum_{i=1}^{N} Y_{i i} \mu_{A_{i}, 2}+3 Y\left(\mu_{A}\right) \sum_{i=1}^{N} Y_{i}^{2} \mu_{A_{i}, 2}+3 Y\left(\mu_{A}\right) \sum_{i=1}^{N} Y_{i} Y_{i i} \mu_{A_{i}, 3}
$$




$$
\begin{aligned}
& +\frac{3}{4} \sum_{i=1}^{N-2} \sum_{j=i+1}^{N-1} \sum_{k=j+1}^{N} Y_{i i} Y_{j j} Y_{k k} \mu_{A_{i}, 2} \mu_{A_{j}, 2} \mu_{A_{k}, 2}+\frac{3}{2} \sum_{i=1}^{N-1} \sum_{j=i+1}^{N} Y_{i i} Y_{i j} Y_{j j} \mu_{A_{i}, 3} \mu_{A_{j}, 3} \\
& +\frac{3}{8} \sum_{i=1}^{N} \sum_{\substack{j=1 \\
j \neq i}}^{N} Y_{i i} Y_{j j}^{2} \mu_{A_{i}, 2} \mu_{A_{j}, 4}+\frac{1}{8} \sum_{i=1}^{N} Y_{i i}^{2} \mu_{A_{i}, 6}+\frac{3}{2} \sum_{i=1}^{N} \sum_{\substack{j=1 \\
j \neq i}}^{N} Y_{i}^{2} Y_{i j} \mu_{A_{i}, 2} \mu_{A_{j}, 2} \\
& +\frac{3}{2} \sum_{i=1}^{N} Y_{i}^{2} Y_{i i} \mu_{A_{i}, 4}+\frac{3}{2} Y\left(\mu_{A}\right) \sum_{i=1}^{N-1} \sum_{j=i+1}^{N} Y_{i i} Y_{j j} \mu_{A_{i}, 2} \mu_{A_{j}, 2}+\frac{3}{4} Y\left(\mu_{A}\right) \sum_{i=1}^{N} Y_{i i}^{2} \mu_{A_{i}, 4} \\
& +\frac{3}{2} \sum_{i=1}^{N} \sum_{\substack{j=1 \\
j \neq i}}^{N} Y_{i} Y_{i i} Y_{j j} \mu_{A_{i}, 3} \mu_{A_{j}, 2}+\frac{3}{4} \sum_{i=1}^{N} Y_{i} Y_{i i}^{2} \mu_{A_{i}, 5} \\
& +\frac{3}{2} \sum_{i=1}^{N} \sum_{\substack{j=1 \\
j \neq i}}^{N-1} \sum_{\substack{k=j+1 \\
k \neq i}}^{N} Y_{i i} Y_{j k}^{2} \mu_{A_{i}, 2} \mu_{A_{j}, 2} \mu_{A_{k}, 2}+\frac{9}{4} \sum_{i=1}^{N-2} \sum_{j=i+1}^{N-1} \sum_{k=j+1}^{N} Y_{i j} Y_{i k} Y_{j k} \mu_{A_{i}, 2} \mu_{A_{j}, 2} \mu_{A_{k}, 2} \\
& +\sum_{i=1}^{N-1} \sum_{j=i+1}^{N} Y_{i j}^{3} \mu_{A_{i}, 3} \mu_{A_{j}, 3}+\frac{3}{2} \sum_{i=1}^{N} \sum_{\substack{j=1 \\
j \neq i}}^{N} Y_{i j}^{2} Y_{j j} \mu_{A_{i}, 2} \mu_{A_{j}, 4}+6 \sum_{i=1}^{N-1} \sum_{j=i+1}^{N} Y_{i} Y_{j} Y_{i j} \mu_{A_{i}, 2} \mu_{A_{j}, 2} \\
& +3 Y\left(\mu_{A}\right) \sum_{i=1}^{N-1} \sum_{j=i+1}^{N} Y_{i j}^{2} \mu_{A_{i}, 2} \mu_{A_{j}, 2}+3 \sum_{i=1}^{N} \sum_{\substack{j=1 \\
j \neq i}}^{N} Y_{i} Y_{i j} Y_{j j} \mu_{A_{i}, 2} \mu_{A_{j}, 3}+3 \sum_{i=1}^{N} \sum_{\substack{j=1 \\
j \neq i}}^{N} Y_{i} Y_{i j}^{2} \mu_{A_{i}, 3} \mu_{A_{j}, 2} \\
& -3 \mu_{Y^{*}} \sigma_{Y^{*}}^{2}-\mu_{Y^{*}}^{3} .
\end{aligned}
$$

We can then use the expression of $\sigma_{Y^{*}}^{2}$ from previous to compute $\boldsymbol{v}_{Y^{*}}=\mu_{Y^{*}, 3} / \sigma_{Y^{*}}^{3}$.

\section{F Relation to ACR}

Arkolakis, Costinot, and Rodríguez-Clare (2012), henceforth ACR, consider an open-economy model with no intermediate inputs and a single factor of production per country. They impose some macro-level restrictions, and prove a powerful characterization of the gains from trade. Namely, they assume that (1) trade is balanced, (2) profits are a constant share of revenues, and (3) import demand system is CES. Using these assumptions, they show that the gains from trade, as measured by the change in real income associated with going to autarky, is given by the reciprocal of the domestic expenditure share raised to the reciprocal of the trade elasticity. The ACR result, and its generalizations (summarized in Costinot and Rodriguez-Clare, 2014), suggest that one can quantify the gains from trade without needing to directly estimate the size of the trade shock.

In Baqaee and Farhi (2019), we show how under certain conditions, changes in iceberg 
trade costs in an open-economy model can be recast as productivity shocks in an associated closed-economy model. This then allows us to use our results to study the second-order effects of trade shocks. For simplicity, we work with a one-factor model (like ACR), but these results can be extended to the case of multiple factors. We also restrict ourselves to nested-CES economies in standard form.

We start by associating a fictitious nested-CES domestic closed-economy model to the true nested-CES open-economy model, both in standard form. The closed economy has the same set $C$ of domestic producers as the open economy and the same elasticities of substitution, but its input-output matrix $\Omega_{i j}^{c} \equiv \Omega_{i j} /\left(\sum_{k \in C} \Omega_{i k}\right)$ is different because each domestic producer only sources from other domestic producers, and not from foreign producers, where $C$ denotes the set of domestic producers.

We show that effects on domestic welfare of a change in trade costs in the true openeconomy model are identical to the effects on aggregate output of a set of productivity shocks $\left(\lambda_{i c} / \bar{\lambda}_{i c}\right)^{1 /\left(1-\theta_{i}\right)}$, where $\lambda_{i c}$ is the domestic cost share of producer $i$ and $\bar{\lambda}_{i c}$ is its steady-state value. It is straightforward to leverage our results to characterize the effects of these shocks up to the second order. Only in some special cases resembling those underpinning our network-irrelevance result in Corollary 1, can a global expression be derived. The baseline ACR specification falls in this category: it has a single sector and no intermediate goods. In this case $\lambda^{c}=1$ and we get $Y^{c} / \bar{Y}^{c}=\left(\lambda_{c} / \bar{\lambda}_{c}\right)^{1 /(1-\theta)}$, where $\lambda_{c}$ is the domestic cost share and $\bar{\lambda}_{c}$ is its steady-state value. 